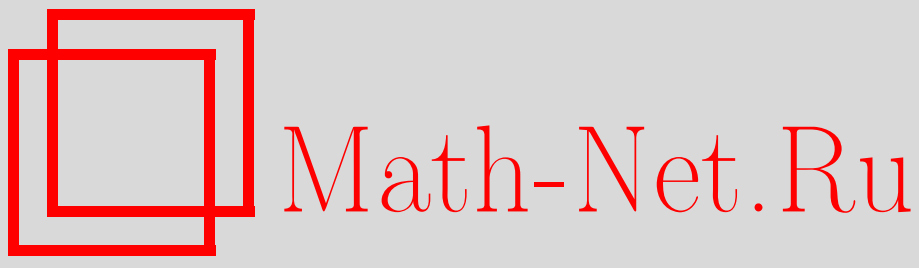

А. С. Кривошеев, Фундаментальный принцип для инвариантных подпространств в выпуклых областях, Изв. РАН. Сер. матем., 2004, том 68, выпуск 2, 71-136

DOI: https://doi.org/10.4213/im476

Использование Общероссийского математического портала Math-Net.Ru подразумевает, что вы прочитали и согласны с пользовательским соглашением

http://www . mathnet.ru/rus/agreement

Параметры загрузки:

IP: 52.90 .164 .192

26 апреля 2023 г., 13:57:34 
УДК 517.5

А. С. Кривошеев

\section{Фундаментальный принцип для инвариантных подпространств в выпуклых областях}

При некоторых естественных ограничениях приведено полное решение задачи интерполяции в пространствах целых функций экспоненциального типа, сопряженная диаграмма которых находится в заданной выпуклой области. Приведено также полное решение проблемы фундаментального принципа для произвольных нетривиальных замкнутых инвариантных относительно дифференцирования и допускающих спектральный синтез подпространств функций, аналитических в выпуклой области.

Библиография: 57 наименований.

\section{$\S 1$. Введение}

Пусть $D$ - выпуклая область в $\mathbb{C}$ и $H(D)$ - пространство функций, аналитических в $D$, с топологией равномерной сходимости на компактных подмножествах из $D$. Пространство $H(D)$ метризуемо, более того, оно является пространством Фреше-Шварца (см., например, [1, гл. 1, теорема 4.6]).

Пусть $\left\{K_{m}\right\}_{m=1}^{\infty}-$ последовательность выпуклых компактов из $D$, исчерпьваюшая $D$, т. е. такая, что $K_{m} \subset \operatorname{int} K_{m+1}, m \geqslant 1$, и $\bigcup_{m} K_{m}=D$ (int $M$ - внутренность множества $M)$. В силу вложения $K_{m} \subset \operatorname{int} K_{m+1}$ для каждого $m \geqslant 1$ найдется $\alpha_{m}>0$ такое, что выполнено неравенство

$$
H_{K_{m}}(z)+\alpha_{m}|z| \leqslant H_{K_{m+1}}(z) \quad \forall z \in \mathbb{C} .
$$

Здесь $H_{M}(z)=\sup _{y \in M} \operatorname{Re} z y$ - опорная функция множества $M$ (точнее, комплексно-сопряженного к $M$ множества); $H_{M}(z)$ выпукла, положительно однородна порядка один, полунепрерьвна снизу, может принимать значение $+\infty$, непрерывна во внутренности того множества, где принимает конечные значения. Если $M$ ограничено, то $H_{M}(z)$ ограничена и непрерывна (см. [2]).

Пусть $H^{*}(D)$ - сильно сопряженное к $H(D)$ пространство (назьваемое $n p o$ странством аналитических функционалов в $D$ ). Известно (см., например, $[1$, гл. $3, \S 12$, п. 7$])$, что преобразование Лапласа аналитических функционалов $\mu \in H^{*}(D)$, задаваемое по формуле

$$
f(z)=(\mu, \exp (z \lambda)), \quad z \in \mathbb{C},
$$

устанавливает алгебраический и топологический изоморфизм между пространством $H^{*}(D)$ и подпространством $\mathbf{P}_{D}$ (используется также обозначение $\left[1, H_{D}\right)$ )

Работа подготовлена при поддержке гранта Президента РФ МД-278.2003.01 и гранта РФФИ № 02-01-01100 и Фонда содействия отечественной науки. 
цельх функций экспоненциального типа (т. е. функций, допускающих оценку вида $|f(z)| \leqslant C \exp (A|z|), z \in \mathbb{C}$, где $C, A$ - некоторые положительные постоянные, зависящие от $f$ ), являющимся индуктивным пределом банаховых пространств:

$$
\begin{gathered}
\mathbf{P}_{D}=\operatorname{limind}_{m \rightarrow \infty} \mathbf{P}_{m}, \\
\mathbf{P}_{m}=\left\{h \in H(\mathbb{C}):\|h\|_{m}=\sup _{z \in \mathbb{C}} h(z) \mid \exp \left(-H_{K_{m}}(z)\right)<\infty\right\} .
\end{gathered}
$$

Пространство $\mathbf{P}_{D}$ есть так называемое $\mathbf{L} \mathbf{N}^{*}$-пространство, т. е. является объединением последовательности банаховых пространств $\mathbf{P}_{m}$ таких, что $\mathbf{P}_{m} \subset P_{m+1}$ для каждого $m=1,2, \ldots$ и эти вложения вполне непрерьвны (см. оценку (1.1)). Следовательно, $\mathbf{P}_{D}$ является отделимым и полным (см., например, [1, гл. $1, \S 2$, теорема 2.4]).

Для субгармонической функции $\psi(z)$ первого порядка и конечного типа $(|\psi(z)| \leqslant a+b|z|)$ через $h_{\psi}(z)$ и $\underline{h}_{\psi}(z)$ обозначим соответственно ее верхний и нижний индикаторы, т. е.

$$
h_{\psi}(z)=\varlimsup_{t \rightarrow+\infty} \frac{\psi(t z)}{t}, \quad \underline{h}_{\psi}(z)=\lim _{\delta \rightarrow 0} \lim _{t \rightarrow+\infty} \frac{1}{\pi \delta^{2}} \int_{B(z, \delta)} \frac{\psi(t y)}{t} d \sigma(y) .
$$

Если $\psi(z)=\ln |f(z)|$, где $f(z)$ - целая функция экспоненциального типа, то вместо $h_{\ln |f|}$ и $\underline{h}_{\ln |f|}$ будем записывать просто $h_{f}$ и $\underline{h}_{f}$. Из определений индикаторов легко следует, что они положительно однородны порядка один и верно неравенство $\underline{h}_{\psi}(z) \leqslant h_{\psi}(z) \forall z$. Если в некоторой точке $z$ выполнено равенство $\underline{h}_{\psi}(z)=h_{\psi}(z)$, то говорят, что $\psi$ (или $f$, если $\psi=\ln |f|$ ) имеет (вполне) регулярныи рост на луче $t z, t>0$. Индикатор $h_{\psi}(z)$ является ограниченной выпуклой, а следовательно, и непрерьвной функцией (см., например, [1]). Отметим, что если $f \in \mathbf{P}_{D}$, то из определений $\mathbf{P}_{D}$ и $h_{f}$ сразу следует, что для некоторого $m \geqslant 1$ выполнено неравенство

$$
h_{f}(z) \leqslant H_{K_{m}}(z)<H_{D}(z) \quad \forall z \in \mathbb{C} \backslash\{0\} .
$$

Обратно, если $h_{f}(z)<H_{D}(z) \forall z \in \mathbb{C} \backslash\{0\}$, то для некоторого $m \geqslant 1$ верна оценка $h_{f}(z) \leqslant H_{K_{m}}(z) \forall z \in \mathbb{C}$. Тогда из теоремы Хартогса о верхнем пределе для семейств субгармонических функций $[1$, гл. $1, \S 6$, теорема 6.1$]$ с учетом (1.1) нетрудно получить также оценку

$$
|f(z)| \leqslant C \exp H_{K_{m+1}}(z) \quad \forall z \in \mathbb{C}
$$

(где $C$-некоторая положительная постоянная), которая означает, что $f \in \mathbf{P}_{D}$. Таким образом, необходимым и достаточным условием принадлежности целой функции $f$ пространству $\mathbf{P}_{D}$ является неравенство

$$
h_{f}(z)<H_{D}(z) \quad \forall z \in \mathbb{C} \backslash\{0\} .
$$

Пусть $W$ - нетривиальное $(W \neq\{0\}, H(D))$ замкнутое подпространство в $H(D)$, инвариантное относительно оператора дифференцирования, т. е. вместе с каждой функцией $\varphi$ оно содержит также и ее производную $\varphi^{\prime}$. Примером такого 
инвариантного подпространства может служить пространство решений из $H(D)$ системы однородных сверточных уравнений (см., например, [1, гл. $3, \S 14$, п. 2]):

$$
\mathbf{M}_{\mu_{i}}[\varphi](z)=\left(\mu_{i}, \varphi(z+y)\right) \equiv 0, \quad \mu_{i} \in H^{*}(D), \quad i=1, \ldots, p .
$$

Частным случаем уравнений свертки являются линейные дифференциальные уравнения с постоянными коэффициентами (как конечного, так и бесконечного порядков), линейные разностные уравнения с постоянными коэффициентами, дифференциально-разностные уравнения, некоторые типы интегральных уравнений и др. Например, дифференциальное уравнение

$$
L(\varphi)=\sum_{k=0}^{s} a_{k} \varphi^{(k)}=0
$$

является уравнением свертки, порожденным функционалом

$$
\mu=\sum_{k=0}^{s}(-1)^{k} a_{k} \delta^{(k)} .
$$

Легко видеть, что характеристический многочлен уравнения (1.3) совпадает с преобразованием Лапласа функционала $\mu$. Пусть $\left\{\lambda_{k}, m_{k}\right\}, k=1, \ldots, l$, $\sum m_{k}=s,-$ последовательность нулей этого многочлена и их кратностей. Хорошо известно, что каждое решение уравнения (1.3) можно записать в виде

$$
\varphi(z)=\sum_{k=1, m=0}^{l, m_{k}-1} c_{k, m} z^{m} \exp \left(\lambda_{k} z\right) .
$$

Возможность такого представления иногда называют фундаментальным приниипом Л. Эйлера для пространств решений однородных дифференциальных уравнений. В этой связи естественно возникает проблема фундаментального принципа, т. е. аналогичного представления решений более общих однородных уравнений свертки и их систем. Сформулируем задачу поточнее. Пусть $f_{1}, \ldots, f_{p}$ - преобразования Лапласа соответственно функционалов $\mu_{1}, \ldots, \mu_{p}$, порождающих систему уравнений (1.2). Функции $f_{1}, \ldots, f_{p}$ называются характеристическими функииями операторов $\mathbf{M}_{\mu_{1}}, \ldots, \mathbf{M}_{\mu_{p}}$. Пусть $\lambda_{k}, k=1,2, \ldots,-$ все обшие нули функций $f_{1}, \ldots, f_{p}$ и $m_{k}-$ их кратности, т. е. для каждого $k=1,2, \ldots$ имеют место равенства

$$
f_{i}^{(n)}\left(\lambda_{k}\right)=0, \quad i=1, \ldots, p, \quad n=0, \ldots, m_{k}-1,
$$

и сушествует $i=i(k)$ такой, что $f_{i}^{\left(m_{k}\right)}\left(\lambda_{k}\right) \neq 0$. Рассмотрим систему

$$
\left\{z^{n} \exp \left(\lambda_{k} z\right)\right\}_{k=1, n=0}^{\infty, m_{k}-1}
$$

В силу выбора показателей $\lambda_{k}$ и определения преобразования Лапласа легко показать (простым дифференцированием равенств (1.2)), что каждая функция из системы (1.4) является решением системы (1.2). Решения вида (1.4) называются әлементарными решениями (1.2). Проблема фундаментального принщипа 
формулируется следующим образом: при каких условиях каждое решение системы (1.2) можно представить в виде абсолютно и равномерно сходящегося на каждом компакте из $D$ ряда элементарных решений:

$$
\varphi(z)=\sum_{k=1, n=0}^{\infty, m_{k}-1} c_{k, n} z^{n} \exp \left(\lambda_{k} z\right) ?
$$

Аналогичная проблема имеет место и для более общих инвариантных подпространств. Поясним, какие функции здесь являются функциями из системы (1.4). Пусть ort $W$ - подпространство в $H^{*}(D)$, состоящее из всех функционалов, обращающихся в нуль на каждом элементе из $W$. Если $W$ не тривиально, то и ort $W$ также не тривиально. Через $I_{W}(D)$ обозначим подпространство в $\mathbf{P}_{D}$, состоящее из преобразований Лапласа всех функционалов из ort $W$. Пусть $\left\{\lambda_{k}, m_{k}\right\}-$ последовательность всех обших нулей и их кратностей функций из $I_{W}(D)$. Легко видеть, что если $W$ - пространство решений системы вида (1.2), то последовательность $\left\{\lambda_{k}, m_{k}\right\}$, определенная выше по функциям $f_{1}, \ldots, f_{p}$, совпадает с аналогичной последовательностью, определенной по всем $f \in I_{W}(D)$. По $\left\{\lambda_{k}, m_{k}\right\}$ построим систему функций (1.4). Каждый функционал $\mu \in \operatorname{ort} W$ обрашается в нуль на этой системе (это можно проверить, дифференцируя равенство из определения преобразования Лапласа). Поэтому любая функция из (1.4) принадлежит $W$ (по следствию из теоремы Хана-Банаха). Проблема фундаментального принщипа для инвариантных подпространств формулируется следующим образом: при каких условиях каждую функцию из $W$ можно разложить в ряд (1.5)?

Заметим, что последовательность $\left\{\lambda_{k}\right\}$ является спектром оператора дифференцирования в подпространстве $W$. При этом $\exp \left(\lambda_{k} z\right)$ являются собственными функциями, а $z^{n} \exp \left(\lambda_{k} z\right), n=1, \ldots, m_{k}-1,-$ присоединенными функциями оператора дифференцирования в $W$. С учетом этого последовательность $\left\{\lambda_{k}, m_{k}\right\}$ иногда называют спектром подпространства $W$.

Задача описания инвариантных подпространств традиционно подразделяется на две подзадачи:

1) проблема спектрального синтеза;

2) проблема фундаментального принщипа.

Первая состоит в нахождении условий, при которых линейная оболочка системы (1.4) плотна в $W$. В случае ее положительного решения говорят, что $W$ допускает спектральный синтез. Вторую проблему, естественно, имеет смысл рассматривать лишь для инвариантных подпространств, допускающих спектральньй синтез. Поэтому в дальнейшем мы будем иметь дело лишь с такими подпространствами. В этой связи отметим следующее. Известно [3], что ядро оператора свертки, т.е. пространство решений одного однородного сверточного уравнения, всегда допускает спектральный синтез. Пространства решений систем однородных уравнений свертки уже не всегда допускают спектральный синтез. Однако имеются простые достаточные условия, а также критерий допустимости спектрального синтеза и в этом случае (см. [4] и [5]). В этих же работах получен критерий допустимости спектрального синтеза и в более общем случае - для произвольных нетривиальных замкнутых инвариантных подпространств.

Первый результат, обобшающий фундаментальный принщип Л. Эйлера на случай уравнений свертки, более общих, чем дифференщиальные уравнения конечного 
порядка с постоянными коэффициентами, был получен Ж. Валироном [6]. Он касается представления целых решений однородного дифференциального уравнения бесконечного порядка с постоянными коэффициентами в виде ряда по элементарным решениям. Этот результат получил дальнейшее развитие в работах [7]-[9] и др. К концу 40-х годов прошлого столетия была замечена тесная связь между проблемой представления решений однородных уравнений свертки и проблемой интерполящии в пространствах целых функций. Оказалось, что эти проблемы двойственные. Первый, кто использовал разрешимость интерполящионной задачи для разложения решений уравнения свертки в ряды экспонент, был, по-видимому, А. $\Phi$. Леонтьев. Вслед за ним указанная связь использовалась уже систематически. Проблема интерполящии в пространствах целых функций сама по себе представляет значительньй интерес и уже давно исследуется. Теория интерполяции аналитическими функциями, развитие которой началось в работах Ньютона и Лагранжа, является важной областью современного анализа. Интерес к этой тематике обусловлен обширной сферой ее приложений в вопросах полноты систем аналитических функций, в теории дифференциальных уравнений, уравнений свертки, краевых задачах, задачах оптимального управления и других областях математики. Вопросами интерполирования в классах целых функций конечного порядка занимались многие математики. Отметим исследования А.О. Гельфонда [10], В.Л. Гончарова [11], М.А. Евграфова [12], Б.Я. Левина [13], А.Ф. Леонтьева [14]-[18], И. И. Ибрагимова [19], И. И. Ибрагимова и М. В. Келдыша [20], Ю. А. Казьмина [21]--[23], Ю. Ф. Коробейника [24], [25] и др.

В общей постановке задача интерполяции состоит в следующем. На линейном пространстве $Q$ заданы линейные функционалы $\mu_{k}, k \geqslant 1$. Для последовательности чисел $\left\{b_{k}\right\}$ требуется найти элемент $f \in Q$, который удовлетворяет условиям

$$
\mu_{k}(f)=b_{k}, \quad k=1,2, \ldots
$$

Пусть в комплексной плоскости задана последовательность попарно различных точек $\left\{\lambda_{k}\right\}_{k=1}^{\infty}$ (узлов интерполяции), $\left|\lambda_{k}\right| \rightarrow+\infty$. Пусть $\mu_{k}, k \geqslant 1,-$ последовательность функционалов, определенных формулами

$$
\mu_{k}(f)=g\left(\lambda_{k}\right), \quad k=1,2, \ldots
$$

Ряд

$$
f(z)=\sum_{k=1}^{\infty} \frac{b_{k} E(z)}{\left(z-\lambda_{k}\right) E^{\prime}\left(\lambda_{k}\right)}
$$

где $E(z)$ - целая функция с простыми нулями в узлах $\lambda_{k}$, называется $p я д о м$ Лагранжа . Он является интерполяционным рядом для задачи (1.6), (1.7). Первый общий результат по проблеме интерполяции в пространствах цельх функций конечного порядка был получен в 1940 г. Б.Я. Левиным [13]. Он рассмотрел задачу (1.6), (1.7) с узлами, образующими регулярное множество (см. [26, гл. 2]). В этом случае вне некоторого исключительного множества функция $E(z)$ удовлетворяет асимптотическому равенству

$$
\ln |E(z)| \approx h(\varphi) r^{\rho(r)}
$$


где $z=r \exp (i \varphi), \rho(r)-$ уточненный порядок функции $E(z)($ см. [26]) и $h(\varphi)-\mathrm{ee}$ индикатор. Это равенство позволило доказать, что при условии

$$
\ln \left|b_{k}\right| \leqslant\left(h\left(\arg \lambda_{k}\right)+\varepsilon\right)\left|\lambda_{k}\right|^{\rho\left(\left|\lambda_{k}\right|\right)} \quad \forall \varepsilon>0, \quad k>k(\varepsilon),
$$

ряд (1.8) сходится равномерно на каждом компакте, а его сумма является целой функцией класса $[\rho(r), h(\varphi)]$, т. е. функцией, индикатор которой меньше или равен $h(\varphi) r^{\rho(r)}$. Далее при решении интерполящионной задачи используются различные обобщения ряда Лагранжа.

В 1948 г. А.Ф. Леонтьев [14] впервые рассмотрел задачу, получившую затем название задачи свободной интерполяиии: определить, каким условиям должна удовлетворять последовательность узлов $\left\{\lambda_{k}\right\}$ для того, чтобы по каждой последовательности чисел $\left\{b_{k}\right\}$, удовлетворяющих неравенству

$$
\ln \left|b_{k}\right| \leqslant\left|\lambda_{k}\right|^{\rho+\varepsilon} \quad \forall \varepsilon>0, \quad k>k(\varepsilon),
$$

можно было построить целую функцию класса $[\rho, \infty]$, т. е. функцию порядка не выше $\rho$ (конечного или бесконечного типа), решающую интерполяционную задачу. А.Ф. Леонтьев, используя обобшенный ряд Лагранжа, полностью решил эту задачу. Ранее некоторые достаточные условия разрешимости задачи (1.6), (1.7) в пространстве $[\rho, \infty]$ нашли М. Мурси, Е. Уинн [27] и А. Макинтайр, Р. Уилсон [28]. А. Ф. Леонтьев рассмотрел также задачу свободной интерполяции в класce $[\rho, \infty)$, т. е. когда числа последовательности $\left\{b_{k}\right\}$ удовлетворяют неравенству $\ln \left|b_{k}\right| \leqslant A+C\left|\lambda_{k}\right|^{\rho}$, а искомая целая функция должна иметь порядок не выше $\rho$ и конечньй тип. Он решил эту задачу для нецелого $\rho$ в работе [15] и в общем случае-в работе [17]. В дальнейшем идеи Б. Я. Левина и А. Ф. Леонтьева развивались в работах [29]-[31]. Полностью задача свободной (простой) интерполяции в классе $[\rho(r), h(\varphi)]$ была решена в работах [32]-[35].

Наряду с простой интерполящией рассматривалась также задача кратной интерполяции, когда в роли функционалов из (1.6) выступают не только $\delta$-функции, но и их производные. Пусть задана последовательность $\left\{\lambda_{k}, m_{k}\right\}$, где $\left|\lambda_{k}\right| \rightarrow+\infty$ и $m_{k}$ - натуральные числа. Для любой последовательности комплексньх чисел $\left\{b_{k, n}\right\}_{k=1, n=1}^{\infty, m_{k}}$ заданного роста требуется найти целую функцию $f(z)$ заданного класса, обладающую дополнительным свойством

$$
f^{(n-1)}\left(\lambda_{k}\right)=b_{k, n}, \quad n=1, \ldots, m_{k}, \quad k=1,2, \ldots
$$

Одним из первых эту задачу в классе $[\rho, \infty]$ при некоторых специальных предположениях относительно узлов интерполяции решил М. Мурси [36]. Г. П. Лапин [37], [38] перенес результаты А. Ф. Леонтьева о свободной интерполящии в классах $[\rho, \infty]$ и $[\rho, \infty)$ на задачу о кратной интерполящии. Дальнейшее свое развитие эта задача получила в работах [39]-[43]. Полностью задача кратной интерполящии в классах $[\rho(r), H(\varphi)]$ была решена в [44] при наличии следуюшего ограничения на кратность $m_{k}$ точек $\lambda_{k}$ :

$$
\left|\lambda_{k}\right|^{-\rho\left(\left|\lambda_{k}\right|\right)} m_{k} \ln \left|\lambda_{k}\right| \rightarrow 0 \text {. }
$$

В работе [46] эта же задача решена при более слабом ограничении на кратность:

$$
\left|\lambda_{k}\right|^{-\rho\left(\left|\lambda_{k}\right|\right)} m_{k} \rightarrow 0
$$


Задача кратной интерполящии в классах $[\rho(r), H(\varphi))$ оказалась, по-видимому, сложнее. В настоящее время известны лишь некоторые достаточные условия для этих классов, а в случае ограниченной функции $H(\varphi)$ и некоторые необходимые условия, не совпадаюшие с достаточными (см. [45], [46]). Критерий для ограниченной функции $H(\varphi)$ получается лишь при дополнительном жестком ограничении на последовательность $\left\{\lambda_{k}, m_{k}\right\}$. В случае неограниченной $H(\varphi)$ ситуация еше сложнее. $\mathrm{K}$ примеру, при $\rho(r) \equiv 1$, т. е. в классах $\mathbf{P}_{D}=\left[1, H_{D}\right)$, критерий разрешимости кратной интерполящионной задачи (впрочем, как и простой) известен лишь для $D=\mathbb{C}$, т. е. в классе $[1, \infty)$ (см. [45]). Сушествует также критерий, когда $D$ является полуплоскостью или полосой, но лишь в случае, когда последовательность $\left\{\lambda_{k}\right\}$ сосредоточена вдоль прямой, перпендикулярной граничным прямым [46].

Отметим еще работу [47], где решена интерполящионная задача в так называемых алгебрах Хермандера, которые являются обобщением пространства $[\rho, \infty)$. Никаких новых результатов по интерполяции для пространств целых функций конечного порядка эта работа не содержит. Однако в ней, одной из первых, предложен новый метод интерполящии, который основан не на интерполяционном ряде Лагранжа или на каких-либо его обобщениях, а на разрешимости $\bar{\partial}$-уравнения с оценками.

Отметим один (на наш взгляд, существенный) общий недостаток всех работ по кратной интерполящии. Это - неудачный выбор классов последовательностей $\left\{b_{k, n}\right\}$, элементы которых стоят в правой части (1.9). Выбор того или иного класса обусловлен оценками на производные $f^{(n-1)}\left(\lambda_{k}\right)$ функций $f \in[\rho(r), H(\varphi)]$ $([\rho(r), H(\varphi)))$. При получении этих оценок использовались неравенства Коши. Последние, безусловно, точные на всем классе целых функций, являются довольно грубыми на классе целых функций конечного порядка, который участвует в интерполящии. Применение неравенств Коши необоснованно расширяет класс правых частей в (1.9), что приводит к искусственным ограничениям на кратность $m_{k}$ точек $\lambda_{k}$. Этих ограничений удалось избежать лишь автору работы [46], но при этом класс правых частей в (1.9) выглядит здесь еше более искусственным, чем в других работах. Выбор такого класса обусловлен, по-видимому, техникой доказательства, используемой в [46]. Далее мы покажем, что при ограничениях на кратность, которые предполагаются в работах предшественников, пространство правых частей может выглядеть более естественным, если оценки, определяющие это пространство, освободить от некоторых “технических" сомножителей.

В настоящей работе мы исследуем кратную интерполящионную задачу в пространствах $\left[1, H_{D}\right)=\mathbf{P}_{D}$. Для ее решения предлагается усовершенствованньй метод, использующий $\bar{\partial}$-технику. Прежде всего, вводится естественное пространство последовательностей $\left\{b_{k, n}\right\}$. Оценки на рост этих последовательностей соответствуют точным оценкам производных целых функций экспоненциального типа, которые выводятся не из общих неравенств Коши, а из интегральных представлений, присущих только таким функциям. При таком же ограничении кратности, как и в [46] $\left(m_{k}\left|\lambda_{k}\right|^{-1} \rightarrow 0\right)$, мы полностью решаем кратную интерполящионную задачу в пространствах $\mathbf{P}_{D}$ для любой (ограниченной или неограниченной) выпуклой области $D$. На этой основе при том же ограничении кратности дается полное решение проблемы фундаментального принципа для произвольных нетривиальных 
замкнутых инвариантных подпространств функций, аналитических в выпуклых областях комплексной плоскости, допускающих спектральный синтез. Однако, в отличие от [46], мы обосновываем указанное ограничение кратности, показывая, что оно необходимо для фундаментального принципа в достаточно большом числе случаев. Отметим еще, что нас не интересовал случай более общих пространств $[\rho(r), H(\varphi))$, так как основной в настоящей работе является проблема фундаментального принципа. По-видимому, преодолев некоторые технические сложности, результат настоящей работы можно перенести и на эти пространства.

Работа состоит из шести параграфов. В $\S 2$ устанавливается эквивалентность разрешимости кратной интерполящионной задачи и фундаментального принщипа. В $\S 3,4$ доказываются соответственно достаточные и необходимые условия интерполящии. В $\S 5$ приводятся различные критерии интерполящии и фундаментального принципа. Наконец, в $\S 6$ рассматриваются некоторые примеры.

\section{§2. Интерполяция и фундаментальный принцип}

Пусть $\left\{\lambda_{k}\right\}$ - последовательность (конечная или бесконечная; в последнем случае $\left.\left|\lambda_{k}\right| \rightarrow \infty\right)$ различных комплексных чисел, $m_{k}, k \geqslant 1,-$ натуральные числа. Рассмотрим кратную интерполящионную задачу

$$
f^{(n-1)}\left(\lambda_{k}\right)=b_{k, n}, \quad n=1, \ldots, m_{k}, \quad k=1,2, \ldots
$$

Найдем, прежде всего, естественные оценки на комплексную последовательность $\left\{b_{k, n}\right\}$ при условии, что функция $f$ принадлежит пространству $\mathbf{P}_{D}$. Пусть $\left\{K_{m}\right\}-$ последовательность выпуклых компактов из области $D$ такая же, как и в начале работы. Для каждых $m, k \geqslant 1$ и $n=1, \ldots, m_{k}$ положим

$$
c_{m, k, n}=\sup _{z \in K_{m}}\left|z^{n-1} \exp \left(z \lambda_{k}\right)\right|
$$

Через $\widetilde{\Phi}$ обозначим индуктивный предел банаховых пространств

$$
\widetilde{\Phi}_{m}=\left\{b=\left\{b_{k, n}\right\}:\|b\|_{m}^{*}=\sup _{k, n}\left|b_{k, n}\right| c_{m, k, n}^{-1}<\infty\right\} .
$$

На пространстве $\mathbf{P}_{D}$ определим линейньй оператор $\widetilde{\Sigma}$ так, что каждой функции $f$ он ставит в соответствие последовательность

$$
b=\left\{b_{k, n}\right\}=\left\{f^{(n-1)}\left(\lambda_{k}\right)\right\} .
$$

Через $\mathbf{S}$ обозначим единичную окружность с центром в нуле.

ЛЕмма 2.1. Для кажддой $f \in \mathbf{P}_{D_{\widetilde{\Sigma}}}$ последовательность $b=\widetilde{\Sigma}(f)$ принадлежит пространству $\widetilde{\Phi}$. Оператор $\widetilde{\Sigma}: \mathbf{P}_{D} \rightarrow \widetilde{\Phi}$ непрерывен. 
ДоКАЗАТЕЛЬСТво. Пусть $f \in \mathbf{P}_{m} \subset \mathbf{P}_{D}$. Из определений индикатора $h_{f}$ и пространства $\mathbf{P}_{m}$ легко следует, что верно неравенство $h_{f}(z) \leqslant H_{K_{m}}(z), z \in \mathbb{C}$. Оно означает, что сопряженная диаграмма функции $f$ (т.е. выпуклый компакт, опорная функция которого совпадает с $h_{f}$ ) лежит на $K_{m}$. Тогда контур $\partial K_{m+1}$, охватьвая компакт $K_{m}$, будет охватывать также и сопряженную диаграмму. Следовательно, имеет место интегральное представление целой функции экспоненциального типа (см. [48, гл. $1, \S 5$, теорема 5.2])

$$
f(\lambda)=\frac{1}{2 \pi i} \int_{\partial K_{m+1}} \exp (z \lambda) \gamma(z) d z
$$

где $\gamma$ - ассоциированная по Борелю функция к функции $f$. Отсюда, дифференцируя под знаком интеграла, для любых $k=1,2, \ldots$ и $n=1, \ldots, m_{k}$ имеем

$$
\left|f^{(n-1)}\left(\lambda_{k}\right)\right| \leqslant(2 \pi)^{-1} \sup _{z \in K_{m+1}}\left|z^{n-1} \exp \left(z \lambda_{k}\right)\right| \sup _{z \in \partial K_{m+1}}|\gamma(z)| l_{m+1},
$$

где $l_{m+1}$ - длина контура $\partial K_{m+1}$. Это неравенство влечет за собой включение $\widetilde{\Sigma}(f) \in \widetilde{\Phi}_{m+1} \subset \widetilde{\Phi}$. Докажем теперь непрерьвность $\widetilde{\Sigma}$. Для этого необходимо оценить $\gamma$ во всех точках контура $\partial K_{m+1}$. Пусть $z_{0} \in \partial K_{m+1}$. Через каждую граничную точку выпуклого компакта $K_{m+1}$ проходит хотя бы одна опорная прямая. Другими словами, найдется $\xi \in \mathbf{S}$ такое, что $H_{K_{m+1}}(\xi)=\operatorname{Re}\left(z_{0} \xi\right)$. В полуплоскости $\left\{y: \operatorname{Re}(y \xi)>h_{f}(\xi)\right\}$ имеет место интегральное представление ассоциированной функции (см. $[48$, гл. $1, \S 5$, теорема 5.3$])$

$$
\gamma(y)=\int_{0}^{+\infty} \xi f(t \xi) \exp (-y t \xi) d t
$$

Поскольку $\operatorname{Re}\left(z_{0} \xi\right)=H_{K_{m+1}}(\xi)>H_{K_{m}}(\xi) \geqslant h_{f}(\xi)$, то оно выполнено и в точке $z_{0}$. Следовательно, с учетом включения $f \in \mathbf{P}_{m} \subset \mathbf{P}_{D}$ и неравенства (1.1) имеем

$$
\begin{aligned}
\left|\gamma\left(z_{0}\right)\right| & \leqslant \int_{0}^{+\infty}|f(t \xi)| \exp \left(\operatorname{Re}\left(-z_{0} t \xi\right)\right) d t \\
& \leqslant\|f\|_{m} \int_{0}^{+\infty} \exp \left(H_{K_{m}}(t \xi)-\operatorname{Re}\left(z_{0} t \xi\right)\right) d t \\
& =\|f\|_{m} \int_{0}^{+\infty} \exp \left(t H_{K_{m}}(\xi)-t H_{K_{m+1}}(\xi)\right) d t \\
& \leqslant\|f\|_{m} \int_{0}^{+\infty} \exp \left(-\alpha_{m} t\right) d t=\|f\|_{m} \alpha_{m}^{-1} .
\end{aligned}
$$

Отсюда и из неравенства (2.1) получаем

$$
\|\widetilde{\Sigma}(f)\|_{m+1}^{*} \leqslant C\|f\|_{m}
$$

где $C>0$ зависит лишь от $m$. Оператор $\widetilde{\Sigma}$ непрерывен на индуктивном пределе $\mathbf{P}_{D}$, если его сужение на любое $\mathbf{P}_{m}$ непрерьвно (см. $[49$, гл. $5, \S 2$, предложение 5]). Так как $\widetilde{\Sigma}$ переводит пространство $\mathbf{P}_{m}$ в $\widetilde{\Phi}_{m+1}$, то непрерывность $\widetilde{\Sigma}: \mathbf{P}_{D} \rightarrow \widetilde{\Phi}$ следует из непрерывности отображения $\widetilde{\Sigma}: \mathbf{P}_{m} \rightarrow \widetilde{\Phi}_{m+1}$, которая имеет место в силу последнего неравенства. Лемма доказана. 
ЗАмечАние 2.2. Рост весовых последовательностей, определяющих пространство $\widetilde{\Phi}$, нельзя уменьшить. Чтобы убедиться в этом, достаточно рассмотреть функции $f(\lambda)=\exp (\lambda z), z \in D$. Действительно, если $z \in K_{m}$ - точка, в которой достигается супремум функции $\left|y^{n-1} \exp \left(y \lambda_{k}\right)\right|$ по компакту $K_{m}$, то верно равенство

$$
\left|f^{(n-1)}\left(\lambda_{k}\right)\right|=\left|z^{n-1} \exp \left(z \lambda_{k}\right)\right|=\sup _{y \in K_{m}}\left|y^{n-1} \exp \left(y \lambda_{k}\right)\right| .
$$

Пусть $\Lambda$-последовательность $\left\{\lambda_{k}, m_{k}\right\}$, а $I_{\Lambda}(D)$ - ядро оператора $\widetilde{\Sigma}: \mathbf{P}_{D} \rightarrow \widetilde{\Phi}$. Тогда $I_{\Lambda}(D)$ - замкнутое подпространство в $\mathbf{P}_{D}$, состоящее из всех тех и только тех функций, которые обращаются в нуль (по крайней мере) в точках $\lambda_{k}$ с кратностью, не менњшей, чем $m_{k}$. В рассматриваемой ситуации можно предполагать, что $I_{\Lambda}(D)$ нетривиально. Действительно, если иметь в виду, что $\Lambda$ - спектр нетривиального инвариантного подпространства, то $I_{\Lambda}(D)$ необходимо содержит нетривиальное подпространство $I_{W}(D)$. Каждая функция $f \in I_{W}(D)$ является преобразованием Лапласа функционала $\nu$, который обращается в нуль на $W$ и, в частности, на элементах системы (1.4). Поэтому, дифференцируя равенство из определения преобразования Лапласа, получаем

$$
f^{(n)}\left(\lambda_{k}\right)=\left(\nu, z^{n} \exp \left(z \lambda_{k}\right)\right)=0, \quad k=1,2, \ldots, \quad n=0,1, \ldots, m_{k}-1,
$$

т. е. $f \in I_{\Lambda}(D)$. Таким образом, в дальнейшем считаем, что $I_{\Lambda}(D) \neq\{0\}$.

Факторпространство $\mathbf{P}_{D} / I_{\Lambda}(D)$, как и $\mathbf{P}_{D}$, является $\mathbf{L} \mathbf{N}^{*}$-пространством и представляет собой объединение возрастающей последовательности банаховых пространств $\widetilde{\mathbf{P}}_{m}$. Элемент $[f] \in \mathbf{P}_{D} / I_{\Lambda}(D)$ принадлежит $\widetilde{\mathbf{P}}_{m}$ тогда и только тогда, когда некоторый представитель $g \in \mathbf{P}_{D}$ класса эквивалентности $[f]$ принадлежит $\mathbf{P}_{m}$. При этом норма $\|[f]\|_{m}$ равна инфимуму норм $\|g\|_{m}$ по всем представителям $g \in \mathbf{P}_{m}$ класса $[f]$. Оператор $\widetilde{\Sigma}$ обычным образом порождает оператор $\Sigma$, действуюший из $\mathbf{P}_{D} / I_{\Lambda}(D)$ в $\widetilde{\Phi}$. Отображение

$$
\Sigma: \mathbf{P}_{D} / I_{\Lambda}(D) \rightarrow \widetilde{\Phi}
$$

является уже инъективным, а также непрерывным в силу леммы 2.1 и определения фактортопологии. Таким образом, верно следующее утверждение.

ПРЕДЛОЖЕНИЕ 2.3. Оператор (2.2) непрерывен и инбективен.

Для последовательности $\Lambda$ и подмножества $E$ окружности $\mathbf{S}$ через $\Lambda(E)$ обозначим подпоследовательность $\left\{\lambda_{k_{j}}, m_{k_{j}}\right\}$ последовательности $\Lambda$, состояшую из всех членов $\Lambda=\left\{\lambda_{k}, m_{k}\right\}$ таких, что $\lambda_{k} /\left|\lambda_{k}\right| \in E$. Положим $m(\Lambda(E))=0$, если $\Lambda(E)$ содержит конечное число элементов, и $m(\Lambda(E))=\varlimsup_{j \rightarrow \infty} m_{k_{j}} /\left|\lambda_{k_{j}}\right|$ в противном случае. Легко видеть, что

$$
m\left(\Lambda\left(E_{1}\right)\right) \leqslant m\left(\Lambda\left(E_{2}\right)\right), \quad E_{1} \subseteq E_{2} .
$$

Если $E=\mathbf{S}$, то вместо $m(\Lambda(E))$ будем записьвать $m(\Lambda)$. Отметим, что каждое $\lambda_{k}$ является нулем (кратности, не меньшей, чем $m_{k}$ ) любой $f \in I_{\Lambda}(D)$. Поскольку $f$ имеет экспоненщиальный тип, то, например, из [48, гл. 1 , теорема 2.3] следует, что $m(\Lambda)<+\infty$. Для выпуклой области $D$ введем множество

$$
J(D)=\left\{z: H_{D}(z)=+\infty\right\} .
$$


Из вьпуклости и однородности функции $H_{D}$ следует, что $\mathbb{C} \backslash J(D)$ является углом раствора не больше $\pi$, за исключением трех следующих случаев:

1) $\mathbb{C} \backslash J(D)$ - вся плоскость, если $D$ ограничена;

2) $\mathbb{C} \backslash J(D)$ - луч, если $D$ - полуплоскость;

3) $\mathbb{C} \backslash J(D)$ - прямая, если $D$ - полоса.

Пусть $F$ - компактное подмножество $\mathbf{S}$. Для $D \neq \mathbb{C}$ положим

$$
m_{F}(\Lambda)=\inf _{E \supset F} m(\Lambda(E)), \quad m_{D}(\Lambda)=\sup _{F \subset(\mathbf{S} \backslash J(D))} m_{F}(\Lambda),
$$

где инфимум берется по всем открытым на $\mathbf{S}$ множествам $E \supset F$, а супремум - по всем компактным подмножествам $F \subset(\mathbf{S} \backslash J(D))$.

Наряду с пространствами $\widetilde{\Phi}_{m}$ и $\widetilde{\Phi}$ рассмотрим еще пространства последовательностей с более простыми весами:

$$
\begin{gathered}
\Phi_{m}=\left\{b=\left\{b_{k, n}\right\}:\|b\|_{m}=\sup _{k, n}\left|b_{k, n}\right| \exp \left(-H_{K_{m}}\left(\lambda_{k}\right)\right)<\infty\right\}, \\
\Phi=\operatorname{limind}_{m \rightarrow \infty} \Phi_{m} .
\end{gathered}
$$

Нетрудно видеть (в силу (1.1)), что вложения $\Phi_{m} \subset \Phi_{m+1}, m=1,2, \ldots$, вполне непрерывны. Поэтому $\Phi$ является $\mathbf{L} \mathbf{N}^{*}$-пространством.

Через $B(x, \delta)$ обозначим круг с центром $x$ радиуса $\delta$.

ПРЕДЛОЖЕНИЕ 2.4. Пусть $m_{D}(\Lambda)=0$. Тогда множества $\Phi$ и $\widetilde{\Phi}$ совпадают, а топологии $\Phi$ и $\widetilde{\Phi}$ эквивалентны.

ДокАЗАТЕЛЬСТво. Покажем, что для каждого $m=1,2, \ldots$ найдутся номер $p=1,2, \ldots$ и постоянная $A>0$ такие, что

$$
\exp \left(H_{K_{m}}\left(\lambda_{k}\right)\right) \leqslant A c_{p, k, n} \quad \forall k=1,2, \ldots, \quad \forall n=1, \ldots, m_{k} .
$$

Предположим, что (2.4) неверно. Тогда для каждого $p=1,2, \ldots$ найдутся $k_{p}=$ $1,2, \ldots$ и $n_{p}=1, \ldots, m_{k_{p}}$ такие, что выполнено неравенство

$$
\exp \left(H_{K_{m}}\left(\lambda_{k_{p}}\right)\right)>p c_{p, k_{p}, n_{p}} .
$$

В силу вложений $K_{p} \subset \operatorname{int} K_{p+1}$ величина $c_{p, k, n}$ возрастает с увеличением $p$ (при неизменных $k$ и $n$ ). Следовательно, если $\left\{\lambda_{k_{p}}\right\}$ содержит бесконечное число одинаковых членов, равных, например, $\lambda_{k}$, то в силу последнего неравенства имеем $\exp \left(H_{K_{m}}\left(\lambda_{k^{\prime}}\right)\right)=+\infty$, что невозможно, поскольку $K_{m}$ - компакт. Таким образом, переходя к подпоследовательности, можно полагать, что $k_{p} \rightarrow \infty$ при $p \rightarrow \infty$. Кроме того, можно также полагать, что $\left\{\lambda_{k_{p}}\left|\lambda_{k_{p}}\right|^{-1}\right\}$ сходится к некоторому $x_{0} \in \mathbf{S}$. Пусть вначале $x_{0} \in J(D)$. Из определений $H_{D}$ и $J(D)$ следует, что для некоторого $z_{0} \in D$ верна оценка

$$
\operatorname{Re}\left(z_{0} x_{0}\right)>\max \left\{1, H_{K_{m}}\left(x_{0}\right)\right\} .
$$

Тогда $\left|z_{0}\right|>1$, а с учетом непрерывности $H_{K_{m}}$ найдется $\delta>0$ такое, что

$$
\operatorname{Re}\left(z_{0} x\right)>H_{K_{m}}(x), \quad x \in B\left(x_{0}, \delta\right) .
$$


Выберем номер $p_{0}$ так, что $\lambda_{k_{p}} /\left|\lambda_{k_{p}}\right| \in B\left(x_{0}, \delta\right)$ для всех $p \geqslant p_{0}$. Пусть $K_{p}$, $p \geqslant p_{0},-$ один из компактов, содержащих $z_{0}$ (такой необходимо существует, поскольку последовательность $\left\{K_{p}\right\}$ исчерпывает $\left.D\right)$. Тогда с учетом положительной однородности опорной функции получаем

$$
\begin{aligned}
c_{p, k_{p}, n_{p}}=\sup _{z \in K_{p}}\left|z^{n_{p}} \exp \left(\lambda_{k_{p}} z\right)\right| & \geqslant\left|z_{0}^{n_{p}} \exp \left(\lambda_{k_{p}} z_{0}\right)\right| \\
& >\exp \left(\operatorname{Re}\left(\lambda_{k_{p}} z_{0}\right)\right)>\exp \left(H_{K_{m}}\left(\lambda_{k_{p}}\right)\right) .
\end{aligned}
$$

Это противоречит неравенству (2.5). Пусть теперь $x_{0} \notin J(D)$ и $z_{0}-$ граничная точка $K_{m+1}$, для которой выполнено равенство $\operatorname{Re}\left(z_{0} x_{0}\right)=H_{K_{m+1}}\left(x_{0}\right)$. Если $z_{0}=0$, то вместо $K_{m+1}$ возьмем компакт $K_{m+2}$, граница которого уже не будет содержать начала координат, так как $K_{m+1} \subset \operatorname{int} K_{m+2}$. В силу неравенства (1.1) найдем $\delta>0$ такое, что

$$
\operatorname{Re}\left(z_{0} x\right)>H_{K_{m}}(x)+\frac{\alpha_{m}|x|}{2}, \quad x \in B\left(x_{0}, \delta\right) .
$$

По условию $m_{D}(\Lambda)=0$. Следовательно, $m_{F}(\Lambda)=0$, где $F=\left\{x_{0}\right\}$. Тогда в силу определения величины $m_{F}(\Lambda)$ и сходимости $\left\{\lambda_{k_{p}}\left|\lambda_{k_{p}}\right|^{-1}\right\}$ к $x_{0}$ для каждого $\varepsilon>0$ найдется номер $p_{0}$ такой, что $m_{k_{p}} \leqslant \varepsilon\left|\lambda_{k_{p}}\right|$ для всех $p>p_{0}$. Фиксируем $\varepsilon>0$ такое, что $\varepsilon \ln \left|z_{0}\right|>-\alpha_{m} / 2$, и $p>\max \left(p_{0}, m+1\right)$ такое, что $\lambda_{k_{p}} /\left|\lambda_{k_{p}}\right| \in B\left(x_{0}, \delta\right)$. Тогда $z_{0} \in K_{p}$ и мы получаем

$$
\begin{aligned}
c_{p, k_{p}, n_{p}} & =\sup _{z \in K_{p}}\left|z^{n_{p}} \exp \left(\lambda_{k_{p}} z\right)\right| \geqslant\left|z_{0}^{n_{p}} \exp \left(\lambda_{k_{p}} z_{0}\right)\right| \\
& =\exp \left(n_{p} \ln \left|z_{0}\right|+\operatorname{Re}\left(\lambda_{k_{p}} z_{0}\right)\right)>\exp \left(-n_{p} \alpha_{m}(2 \varepsilon)^{-1}+\operatorname{Re}\left(\lambda_{k_{p}} z_{0}\right)\right) \\
& >\exp \left(-2^{-1} \alpha_{m}\left|\lambda_{k_{p}}\right|+H_{K_{m}}\left(\lambda_{k_{p}}\right)+2^{-1} \alpha_{m}\left|\lambda_{k_{p}}\right|\right)=\exp \left(H_{K_{m}}\left(\lambda_{k_{p}}\right)\right) .
\end{aligned}
$$

Это снова противоречит неравенству (2.5).

Таким образом, неравенство (2.4) выполнено. Используя его, получаем

$$
\|b\|_{p}^{*}=\sup _{k, n}\left|b_{k, n}\right| c_{p, k, n}^{-1} \leqslant A \sup _{k, n}\left|b_{k, n}\right| \exp \left(-H_{K_{m}}\left(\lambda_{k}\right)\right)=A\|b\|_{m} \quad \forall b \in \Phi_{m} .
$$

Отсюда следует, что $\Phi$ вложено в $\widetilde{\Phi}$ и это вложение непрерывно.

Остается показать, что имеет место обратное вложение и оно также непрерывно. Для этого достаточно установить неравенство

$$
c_{m, k, n} \leqslant C \exp \left(H_{K_{p}}\left(\lambda_{k}\right)\right) \quad \forall k=1,2, \ldots, \quad \forall n=1, \ldots, m_{k},
$$

где $p$ зависит только от $m$. Предположим противное. Тогда

$$
c_{m, k_{p}, n_{p}}>p \exp \left(H_{K_{p}}\left(\lambda_{k_{p}}\right)\right)
$$

Как и вьше, можно полагать, что $k_{p} \rightarrow \infty$ при $p \rightarrow \infty$ и $\left\{\lambda_{k_{p}} /\left|\lambda_{k_{p}}\right|\right\}$ сходится к некоторой точке $x_{0} \in \mathbf{S}$. Пусть $x_{0} \in J(D)$. Через $y_{0}$ обозначим точку компакта $K_{m}$ с максимальным модулем. Тогда

$$
c_{m, k_{p}, n_{p}} \leqslant\left|y_{0}\right|^{n_{p}} \exp \left(H_{K_{m}}\left(\lambda_{k_{p}}\right)\right) \leqslant \exp \left(m_{k_{p}}|\ln | y_{0}||+H_{K_{m}}\left(\lambda_{k_{p}}\right)\right) .
$$


Как уже отмечалось ранее, величина $m(\Lambda)$ конечна. Следовательно, $m_{k} \leqslant \beta\left|\lambda_{k}\right|$, $k=1,2, \ldots$. Таким образом,

$$
c_{m, k_{p}, n_{p}} \leqslant \exp \left(\beta\left|\lambda_{k_{p}}\right||\ln | y_{0}||+H_{K_{m}}\left(\lambda_{k_{p}}\right)\right) .
$$

Согласно определениям функции $H_{K_{m}}$ и множества $J(D)$ найдем $z_{0} \in D$ такое, что

$$
\operatorname{Re}\left(z_{0} x_{0}\right)>\beta|\ln | y_{0}||+H_{K_{m}}\left(x_{0}\right) .
$$

По непрерывности эта оценка продолжается в некоторую окрестность $x_{0}$ :

$$
\operatorname{Re}\left(z_{0} x\right)>\beta|\ln | y_{0}|||x|+H_{K_{m}}(x), \quad x \in B\left(x_{0}, \delta\right) .
$$

Выберем номер $p$ так, что компакт $K_{p}$ содержит $z_{0}$ и $\lambda_{k_{p}} /\left|\lambda_{k_{p}}\right| \in B\left(x_{0}, \delta\right)$. Отсюда и из однородности опорной функции получаем

$$
H_{K_{p}}\left(\lambda_{k_{p}}\right) \geqslant \operatorname{Re}\left(z_{0} \lambda_{k_{p}}\right)>\beta\left|\lambda_{k_{p}}\right||\ln | y_{0}||+H_{K_{m}}\left(\lambda_{k_{p}}\right) \geqslant \ln c_{m, k_{p}, n_{p}} .
$$

Это противоречит неравенству (2.9). Случай $x_{0} \notin J(D)$ разбирается аналогично. Предложение доказано.

ЗАмЕчАнИЕ 2.5. В [46] при определении пространств последовательностей, аналогичных $\Phi$, в роли $c_{m, k, n}$ выступают величины $n !\left|\lambda_{k}\right|^{-n} \exp \left(H_{K_{m}}\left(\lambda_{k}\right)\right)$. При этом налагается ограничение на кратность, более жесткое (для неограниченной области $D$ ), чем в настоящей работе: $m(\Lambda)=0$. Это ограничение обеспечивает изоморфность пространства последовательностей из [46] и пространства $\Phi$. Чтобы показать это, достаточно установить оценки

$$
\exp \left(-\varepsilon(k)\left|\lambda_{k}\right|\right) \leqslant\left|\lambda_{k}\right|^{-n} n ! \leqslant \exp \left(\varepsilon(k)\left|\lambda_{k}\right|\right),
$$

где $\varepsilon(k) \rightarrow 0$, когда $k \rightarrow \infty$. Имеем

$$
\frac{n !}{\left|\lambda_{k}\right|^{n}} \leqslant \frac{n^{n}}{\left|\lambda_{k}\right|^{n}} \leqslant\left(\frac{m_{k}}{\left|\lambda_{k}\right|}\right)^{n} \leqslant\left(\frac{\beta\left|\lambda_{k}\right|}{\left|\lambda_{k}\right|}\right)^{n} \leqslant \beta^{m_{k}} \leqslant \exp \left(\varepsilon(k)\left|\lambda_{k}\right|\right) .
$$

Последнее неравенство здесь следует из того, что $m(\Lambda)=0$. Кроме того, как и в предложении 2.4 , мы пользуемся оценкой $m_{k} \leqslant \beta\left|\lambda_{k}\right|$ (где, не ограничивая обшности, считаем, что $\beta>1$ ). Получаем правое неравенство из (2.10). Докажем теперь левое. Учитьвая то, что $m(\Lambda)=0$ и функция $x^{-1} \ln (3 x)$ убывает при $x>1$, имеем

$$
\left|\lambda_{k}\right|^{-1} \ln \left(\frac{\left|\lambda_{k}\right|^{n}}{n !}\right) \leqslant\left|\lambda_{k}\right|^{-1} \ln \left(\frac{3^{n}\left|\lambda_{k}\right|^{n}}{n^{n}}\right)=n\left|\lambda_{k}\right|^{-1} \ln \left(\frac{3\left|\lambda_{k}\right|}{n}\right) \leqslant a_{k}^{-1} \ln \left(3 a_{k}\right) \rightarrow 0,
$$

где $a_{k}=\left|\lambda_{k}\right| / m_{k} \rightarrow+\infty$. Отсюда следует левое неравенство из (2.10).

$\mathrm{B}$ других работах по кратной интерполяции в качестве $c_{m, k, n}$ используют величины $n ! \exp \left(H_{K_{m}}\left(\lambda_{k}\right)\right)$. При этом предполагается следуюшее более жесткое ограничение на кратность: $\left|\lambda_{k}\right|^{-1} m_{k} \ln \left|\lambda_{k}\right| \rightarrow 0$. Возникаюшие при этом пространства последовательностей также изоморфны $\Phi$. В самом деле, верны оценки

$$
\left|\lambda_{k}\right|^{-1} \ln n ! \leqslant\left|\lambda_{k}\right|^{-1} \ln \left(m_{k} !\right) \leqslant\left|\lambda_{k}\right|^{-1} m_{k} \ln m_{k} \leqslant\left|\lambda_{k}\right|^{-1} m_{k} \ln \left(\beta\left|\lambda_{k}\right|\right) \rightarrow 0 .
$$


Отсюда следует, что $1 \leqslant n ! \leqslant \exp \left(\varepsilon(k)\left|\lambda_{k}\right|\right)$, где $\varepsilon(k) \rightarrow 0$. Последнее и дает нам требуемый изоморфизм.

Таким образом, во всех работах предшественников по кратной интерполяции использовались пространства последовательностей, изоморфные пространству $\Phi$, которое, безусловно, является более простым и естественным.

Обратимся теперь к представлению функций из инвариантного подпространства $W$ со спектром $\Lambda$. Прежде всего, заметим, что если функция $\varphi \in W$ представляется рядом, сходяшимся в топологии пространства $H(D)$ :

$$
\varphi(z)=\sum_{k=1, n=0}^{\infty, m_{k}-1} d_{k, n} z^{n} \exp \left(\lambda_{k} z\right),
$$

то это представление единственное. Действительно, если имеется еще одно представление, то, вычитая одно из другого, получаем

$$
0 \equiv \sum_{k=1, n=0}^{\infty, m_{k}-1} d_{k, n}^{\prime} z^{n} \exp \left(\lambda_{k} z\right)
$$

причем ряд сходится в $H(D)$. Пусть $\nu_{k, n}, k=1,2, \ldots, n=1, \ldots, m_{k},-$ биортогональная к системе (1.4) последовательность функционалов из $H^{*}(D)$, т.е. $\nu_{k, n}\left(z^{p-1} \exp \left(\lambda_{j} z\right)\right)=1$ при $k=j, n=p$ и $\nu_{k, n}\left(z^{p-1} \exp \left(\lambda_{j} z\right)\right)=0$ в противном случае. Тогда в силу линейности и непрерывности функционалов $\nu_{k, n}$ имеем

$$
0=\nu_{j, i}(0)=\lim _{p \rightarrow \infty} \sum_{k=1, n=0}^{p, m_{k}-1} d_{k, n}^{\prime} \nu_{j, i}\left(z^{n} \exp \left(\lambda_{k} z\right)\right)=d_{j, i}^{\prime} .
$$

Это противоречит тому, что разложения функции $\varphi$ были разными. Таким образом, имеет место следующее утверждение.

ЛЕмма 2.6. Если $\varphi \in W$ представляется рядом (2.11), сходящимся в топологии $H(D)$, то представление единственное, а его коэффициенты можно вичислить по формуле $d_{k, n}=\nu_{k, n}(\varphi)$, где $\left\{\nu_{k, n}\right\}-$ произвольная последовательность функиионалов из $H^{*}(D)$, биортогональная к системе (1.4)

Указанная последовательность функционалов всегда существует. Для ее построения проще всего найти сначала преобразования Лапласа $\left\{f_{k, n}\right\}$ этих функционалов. Функции $f_{k, n}$ можно построить, например, следуюшим образом. Фиксируем $k \geqslant 1$ и $n=1, \ldots, m_{k}$. Рассмотрим какую-нибудь функцию $f \in I_{W}(D)$, для которой $f^{\left(m_{k}\right)}\left(\lambda_{k}\right) \neq 0$ (такая $f$ существует в силу выбора $\Lambda$ ). Положим

$$
f_{s}(\lambda)=\frac{f(\lambda) m_{k} !}{\left(\lambda-\lambda_{k}\right)^{m_{k}-n+1-s}(s+n-1) ! f^{\left(m_{k}\right)}\left(\lambda_{k}\right)}, \quad s=0, \ldots, m_{k}-n .
$$

Функция $f_{s}(\lambda)$ обрашается в нуль в точке $\lambda_{k}$ с кратностью $s+n-1$, и выполнено равенство $f_{s}^{(s+n-1)}\left(\lambda_{k}\right)=1$. Кроме того, $f_{s}(\lambda)$ обращается в нуль в точках $\lambda_{p}$ с кратностью, не меньшей, чем $m_{p}$, для всех $p \neq k$. Положим далее

$$
\begin{gathered}
\tilde{f}_{1}(\lambda)=f_{0}(\lambda)-\left(f_{0}^{(n)}\left(\lambda_{k}\right)\right)^{-1} f_{1}(\lambda), \\
\tilde{f}_{s}(\lambda)=\tilde{f}_{s-1}(\lambda)-\left(\tilde{f}_{s-1}^{(s+n-1)}\right)^{-1}\left(\lambda_{k}\right) f_{s}(\lambda), \quad s=2, \ldots, m_{k}-n .
\end{gathered}
$$


Тогда функция $f_{k, n}(\lambda)=\tilde{f}_{m_{k}-n}(\lambda)$ обрашается в нуль в точках $\lambda_{p}$ с кратностью, не меньшей, чем $m_{p}$, для всех $p \neq k$. Кроме того, $f_{k, n}^{(p)}\left(\lambda_{k}\right)=0$ для всех $p=$ $0, \ldots, n-2, n, \ldots, m_{k}-1$ и $f_{k, n}^{(n-1)}\left(\lambda_{k}\right)=1$. Таким образом, функция $f_{k, n}$ искомая.

Определим функционал $\nu_{k, n} \in H^{*}(D)$ по формуле

$$
\left(\nu_{k, n}, g\right)=\frac{1}{2 \pi i} \int_{\Delta} g(z) \gamma_{k, n}(z) d z, \quad g \in H(D)
$$

где $\gamma_{k, n}(z)$ - функция, ассоциированная по Борелю с $f_{k, n}$, и $\Delta \subset D$ - контур, охватывающий сопряженную диаграмму функции $f_{k, n}$, или, что то же самое, выпуклую оболочку особенностей $\gamma_{k, n}$. Согласно интегральному представлению целой функции экспоненциального типа, которое мы уже использовали в лемме 2.1, преобразование Лапласа функционала $\nu_{k, n}$ совпадает с функцией $f_{k, n}$. Следовательно,

$$
\nu_{k, n}\left(z^{p-1} \exp \left(\lambda_{j} z\right)\right)=f_{k, n}^{(p-1)}\left(\lambda_{j}\right) .
$$

Из построения $f_{k, n}$ вытекает, что $\left\{\nu_{k, n}\right\}$ - последовательность, биортогональная к (1.4).

Перейдем теперь к описанию последовательностей коэффициентов $\left\{d_{k, n}\right\}$, для которых в области $D$ сходится ряд

$$
\sum_{k=1, n=0}^{\infty, m_{k}-1} d_{k, n} z^{n} \exp \left(\lambda_{k} z\right)
$$

Для каждого $m=1,2, \ldots$ введем банахово пространство

$$
Q_{m}=\left\{d=\left\{d_{k, n}\right\}:\|d\|_{m}=\sup _{k, n}\left|d_{k, n}\right| \exp \left(H_{K_{m}}\left(\lambda_{k}\right)\right)<\infty\right\}
$$

Пусть $Q=\bigcap_{m} Q_{m}$. В пространстве $Q$ определим метрику по формуле

$$
\rho\left(d, d^{\prime}\right)=\sum_{m=1}^{\infty} 2^{-m} \min \left(1,\left\|d-d^{\prime}\right\|_{m}\right) .
$$

Нетрудно видеть, что с этой метрикой $Q$ становится пространством Фреше.

ПРЕДЛОЖЕНИЕ 2.7. Пусть $m_{D}(\Lambda)=0$. Тогда верны утверждения:

1) если ряд (2.12) сходится в каждой точке области $D, \bmod d=\left\{d_{k, n}\right\} \in Q$;

2) для каждого $m=1,2, \ldots$ найдутся номер $p=1,2, \ldots$ и постоянная $C_{0}>0$ такие, что

$$
\sum_{k=1, n=0}^{\infty, m_{k}-1}\left|d_{k, n}\right| c_{m, k, n} \leqslant C_{0}\|d\|_{p} \quad \forall d=\left\{d_{k, n}\right\} \in Q .
$$


ДокаЗАТЕльство. 1) Предположим, что $d \notin Q$. Тогда для некоторого $m=$ $1,2, \ldots$ найдется подпоследовательность $\left\{d_{k_{p}, n_{p}}\right\}$ такая, что

$$
\left|d_{k_{p}, n_{p}}\right| \exp \left(H_{K_{m}}\left(\lambda_{k_{p}}\right)\right)>1
$$

Переходя еше раз к подпоследовательности, можно считать, что $\lambda_{k_{p}} /\left|\lambda_{k_{p}}\right|$ сходится к некоторой точке $x_{0} \in \mathbf{S}$. Тогда, как и при получении оценок $(2.6)$ и $(2.7)$ в предложении 2.4 , найдутся точка $z_{0} \in D$ и номер $p_{0}$ такие, что

$$
\left|z_{0}^{n_{p}} \exp \left(\lambda_{k_{p}} z_{0}\right)\right|>\exp \left(H_{K_{m}}\left(\lambda_{k_{p}}\right)\right), \quad p \geqslant p_{0}
$$

Следовательно, с учетом предыдущего неравенства получаем

$$
\left|d_{k_{p}, n_{p}}\right|\left|z_{0}^{n_{p}} \exp \left(\lambda_{k_{p}} z_{0}\right)\right|>\left|d_{k_{p}, n_{p}}\right| \exp \left(H_{K_{m}}\left(\lambda_{k_{p}}\right)\right)>1
$$

Это противоречит сходимости ряда $(2.12)$ в точке $z_{0} \in D$. Таким образом, $d \in Q$.

2) Пусть $d=\left\{d_{k, n}\right\} \in Q$. В силу неравенства (2.8) с учетом (1.1) имеем

$$
\begin{aligned}
& \sum_{k=1, n=0}^{\infty, m_{k}-1}\left|d_{k, n}\right| c_{m, k, n} \leqslant C \sum_{k=1, n=0}^{\infty, m_{k}-1}\left|d_{k, n}\right| \exp \left(H_{K_{p}}\left(\lambda_{k}\right)\right) \\
& \quad \leqslant C \sum_{k=1, n=0}^{\infty, m_{k}-1}\left|d_{k, n}\right| \exp \left(H_{K_{p+1}}\left(\lambda_{k}\right)+H_{K_{p}}\left(\lambda_{k}\right)-H_{K_{p+1}}\left(\lambda_{k}\right)\right) \\
& \quad \leqslant C\|d\|_{p+1} \sum_{k=1}^{\infty} m_{k} \exp \left(-\alpha_{p}\left|\lambda_{k}\right|\right)
\end{aligned}
$$

Пусть $\left\{\lambda_{s}^{\prime}\right\}$ - перенумерованная в порядке неубывания абсолютных величин последовательность $\left\{\lambda_{k}\right\}$, причем точка $\lambda_{k}$ повторяется в ней столько раз, какова ее кратность, и $f \in I_{\Lambda}(D)$. Поскольку функция $f$ имеет экспоненциальный тип и обрашается в нуль в точках $\lambda_{k}$ с кратностью, не меньшей, чем $m_{k}$, то в силу [48, гл. 1 , теорема 2.3] для некоторого $A>0$ будет выполнено неравенство $s \leqslant A\left|\lambda_{s}^{\prime}\right|$, которое влечет за собой сходимость ряда $\sum_{s} \exp \left(-\alpha_{p}\left|\lambda_{s}^{\prime}\right|\right)$, а вместе с ним и последнего ряда в (2.14). Предложение доказано.

Как следствие этого предложения, можно получить простую реализацию подпространства $W$, любой элемент которого разлагается в топологии $H(D)$ в ряд (2.12). Пусть $\mathbf{E}$ - оператор, который каждой $\varphi \in W$ ставит в соответствие последовательность $d=\left\{\nu_{k, n}(\varphi)\right\}$, где $\left\{\nu_{k, n}\right\} \subset H^{*}(D)$ биортогональна к системе (1.4). Отметим, что в обшем случае (для подпространств, не допускающих спектральный синтез) оператор $\mathbf{E}$ зависит от $\left\{\nu_{k, p}\right\}$.

СЛЕДСТВИЕ 2.8. Пусть $m_{D}(\Lambda)=0$. Тогда әквивалентны утверждения:

1) каждая функиия $\varphi$ из замкнутого подпространства $W$ разлагается в ряд (2.11), который сходится в топологии пространства $H(D)$;

2) отображсене $\mathbf{E}$ устанавливает изоморфизм линейных топологических пространств $W$ и $Q$. 
ДоКАЗАТЕЛЬСТво. 1) $\rightarrow$ 2). Пусть $\varphi \in W$. По условию она разлагается в ряд (2.11), сходящийся в $H(D)$. Тогда по лемме 2.6

$$
\mathbf{E}(\varphi)=\left\{\nu_{k, n}(\varphi)\right\}=\left\{d_{k, n}\right\},
$$

а в силу утверждения 1$)$ предложения $2.7\left\{d_{k, n}\right\} \in Q$. Поскольку разные функции имеют разные коэффициенты разложения, то оператор $\mathbf{E}: W \rightarrow Q$ инъективен. Пусть теперь $d=\left\{d_{k, n}\right\} \in Q$. Тогда в силу утверждения 2) предложения 2.7 ряд (2. 12) сходится в $D$ равномерно на компактах к некоторой функции $\varphi \in H(D)$. Поскольку подпространство $W$ замкнуто и содержит все функции системы (1.4), то $\varphi \in W$. Применяя снова лемму 2.6, получаем $\left\{d_{k, n}\right\}=\left\{\nu_{k, n}(\varphi)\right\}=\mathbf{E}(\varphi)$. Таким образом, оператор $\mathbf{E}$ сюръективен. Из (2.13) следует, что для каждого $m \geqslant 1$

$$
\sup _{z \in K_{m}}|\varphi(z)| \leqslant C_{0}\|d\|_{p} \quad \forall d=\left\{d_{k, n}\right\} \in Q
$$

где $p$ и $C_{0}$ зависят лишш от $m$. Это означает, что оператор $\mathbf{E}^{-1}$ непрерьвен. Тогда по теореме об открытом отображении для пространств Фреше (см. [49, гл. VI, п. 3, теорема 8]) $\mathbf{E}$ является изоморфизмом.

$2) \rightarrow 1)$. Пусть $\varphi \in W$. По условию сушествует $d=\left\{d_{k, n}\right\} \in Q$ такое, что $\mathbf{E}(\varphi)=\left\{d_{k, n}\right\}$. Как и в первой части доказательства, ряд (2.12) сходится равномерно на компактах из $D$ к некоторой функции $\varphi^{\prime} \in W$, причем $\left\{d_{k, n}\right\}=$ $\left\{\nu_{k, n}\left(\varphi^{\prime}\right)\right\}=\mathbf{E}\left(\varphi^{\prime}\right)$. Поскольку $\mathbf{E}-$ биекция, то $\varphi^{\prime}=\varphi$. Следствие доказано.

При разложении функций из $W$ в ряд (2.11) естественно возникает вопрос о выборе топологии, в которой должен сходиться этот ряд. Следующее утверждение показывает, что этот вопрос несушествен: слабая сходимость ряда (2.11) влечет за собой его сильную сходимость, более того, его абсолютную сходимость и специальную равномерную оценку ряда из абсолютных величин.

СлЕДСТВИЕ 2.9. Пусть $m_{D}(\Lambda)=0$. Если любая функиия $\varphi$ замкнутого подпространства $W$ разлагается в ряд (2.11), который сходится в каждой точке области $D$, то для любого $m=1,2, \ldots$ выполнено неравенство

$$
\sum_{k=1, n=0}^{\infty, m_{k}-1}\left|d_{k, n}\right| \sup _{z \in K_{m}}\left|z^{n-1} \exp \left(\lambda_{k} z\right)\right| \leqslant A \sup _{y \in K_{q}}|\varphi(y)|, \quad \varphi \in W
$$

где номер $q$ и постоянная $A>0$ зависят лишь от $m$.

ДоКАЗАТЕЛЬСТво. Изоморфизм между $W$ и $Q$ означает, в частности, что для каждого $p \geqslant 1$ верна оценка

$$
\|\mathbf{E}(\varphi)\|_{p}=\|d\|_{p} \leqslant A_{0} \sup _{y \in K_{q}}|\varphi(y)|, \quad \varphi \in W
$$

где номер $q$ и $A>0$ зависят лиш от $p$. С учетом этого неравенства требуемое утверждение сразу следует из предложения 2.7 и следствия 2.8 . 
ЗАМЕЧАНИЕ 2.10. Базис (1.4), для которого вьполнено (2.15), имеет несколько названий: базис Kете, регулярный базис и др.

Перейдем теперь к установлению эквивалентности разрешимости интерполяционной задачи и проблемы фундаментального принципа. Первым шагом в этом направлении будет следующее утверждение.

Лемма 2.11. Пусть система (1.4) является базисом Кете (т. е. выполнено неравенство (2.15)) в подпространстве $W \subset H(D)$. Тогда оператор $(2.2)$ сюрвективен.

ДокаЗАТЕльство. Пусть $b=\left\{b_{k, n}\right\} \in \widetilde{\Phi}_{D}$. Выберем номер $m=1,2, \ldots$ такой, что $b \in \widetilde{\Phi}_{m}$. Тогда для некоторого $B>0$ имеем

$$
\left|b_{k, n}\right| \leqslant B c_{m, k, n}, \quad k=1,2, \ldots, \quad n=1, \ldots, m_{k}
$$

Поэтому в силу (2.15) для каждой функции $\varphi \in W$ сходится ряд $\sum_{k, n} d_{k, n} b_{k, n}$ $\left(\right.$ где $d_{k, n}-$ коэффициенты разложения $\varphi$ ) и верна оценка

$$
\sum_{k=1, n=0}^{\infty, m_{k}-1}\left|d_{k, n}\right|\left|b_{k, n}\right| \leqslant B A \sup _{y \in K_{q}}|\varphi(y)|,
$$

где $q$ и $A>0$ зависят лишь от $m$. Таким образом, на $W$ определен линейный функционал $\nu(\varphi)=\sum_{k, n} d_{k, n} b_{k, n}$. По теореме Хана-Банаха его можно продолжить на все пространство $H(D)$ так, что будет выполнено неравенство

$$
|\nu(\varphi)| \leqslant B A \sup _{y \in K_{q}}|\varphi(y)|, \quad \varphi \in H(D) .
$$

Отсюда сразу следует, что $\nu$ непрерывен на $H(D)$. Пусть $f$ - преобразование Лапласа функционала $\nu$. Тогда, как уже отмечалось вьше, $f^{(n)}\left(\lambda_{k}\right)=\nu\left(z^{n} \exp \left(\lambda_{k} z\right)\right)$. $\mathrm{C}$ другой стороны, по определению $\nu$ имеем $\nu\left(z^{n} \exp \left(\lambda_{k} z\right)\right)=b_{k, n}$. Это означает, что $\widetilde{\Sigma}(f)=b$. Лемма доказана.

Выше были введены два пространства $I_{W}(D)$ и $I_{\Lambda}(D)$ функций, которые обращаются в нуль в точках $\lambda_{k}$ с кратностью, не меньшей, чем $m_{k}$. В общем случае эти подпространства не совпадают. Однако имеет место следующая

ЛЕмма 2.12. Пусть $W$ - нетривиальное замкнутое инвариантное подпространство в $H(D)$ со спектром $\Lambda$. Следующие утверждения әквивалентны:

1) подпространство $W$ допускает спектральный синтез;

2) $I_{W}(D)=I_{\Lambda}(D)$.

ДокАЗАТЕЛьство. По определению последовательности $\Lambda=\left\{\lambda_{k}, m_{k}\right\}$ каждая функция из $I_{W}(D)$ обращается в нуль в точках $\lambda_{k}$ с кратностью, не меньшей, чем $m_{k}$. Пространство $I_{\Lambda}(D)$ содержит все такие функции. Поэтому верно вложение $I_{W}(D) \subset I_{\Lambda}(D)$. Пусть $W$ допускает спектральный синтез, $f \in I_{\Lambda}(D)$ и $\nu-$ функционал, преобразование Лапласа которого равно $f$. Поскольку $f$ обрашается в нуль в точках $\lambda_{k}$ с кратностью, не меньшей, чем $m_{k}$, то функционал $\nu$ обращается в нуль на всех функциях системы (1.4), так как $f^{(n)}(\lambda)=\left(\nu, z^{n} \exp (\lambda z)\right)$. По предположению линейная оболочка системы (1.4) плотна в $W$. Поэтому $\nu$ обрашается 
в нуль на всем $W$. Тогда по определению пространства $I_{W}(D)$ функция $f$ принадлежит ему. Таким образом, $I_{W}(D)=I_{\Lambda}(D)$.

Обратно, пусть $I_{W}(D)=I_{\Lambda}(D)$. Тогда любая функция, которая обрашается в нуль в точках $\lambda_{k}$ с кратностью, не меньшей, чем $m_{k}$, принадлежит $I_{W}(D)$. Другими словами, каждый функционал, который обращается в нуль на всех функциях системы (1.4), обрашается в нуль и на всем подпространстве $W$. Это означает, что линейная оболочка системы (1.4) плотна в $W$, т. е. $W$ допускает спектральный синтез. Лемма доказана.

В заключение докажем заявленную выше эквивалентность двух задач. Если $Y$ - подпространство $X$ и $x \in X$, то, как и выше, $[x]$ обозначает класс эквивалентности из факторпространства $X / Y$, порожденный $x$.

ПРЕДЛОЖЕНИЕ 2.13. Пусть $W$ - нетривиальное замкнутое инвариантное подпространство в $H(D)$ со спектром $\Lambda$, допускающее спектральньи синтез, и $m_{D}(\Lambda)=0$. Следующие утверждения әквивалентны:

1) оператор $\Sigma: \mathbf{P}_{D} / I_{\Lambda}(D) \rightarrow \Phi$ является изоморфизмом линейных топологических пространств;

2) каждая функиия $\varphi \in W$ представляется рядом (2.11), который сходится в каждой точке области D.

ДокАЗАТЕльство. 2) $\rightarrow$ 1). По предложениям 2.4 и 2.3 оператор $\Sigma:$ $\mathbf{P}_{D} / I_{\Lambda}(D) \rightarrow \Phi$ непрерывен и инъективен, а в силу следствия 2.9 и леммы 2.11 этот оператор является сюръективным. Тогда по теореме об открытом отображении (см. [49, приложение 1, теорема 2]) для отделимых пространств, покрываемых счетным семейством своих подпространств Фреше (каковыми, очевидно, являются $\mathbf{L} \mathbf{N}^{*}$-пространства), оператор $\Sigma$ является изоморфизмом линейных топологических пространств.

$1) \rightarrow 2)$. Поскольку $H(D)$ - пространство Фреше-Шварца, то имеет место изоморфизм (см. [50]): $W \cong\left[H^{*}(D) / \text { ort } W\right]^{*}$. Так как $W$ допускает спектральный синтез, то по лемме $2.12 \mathbf{P}_{D} / I_{W}(D)$ совпадает с $\mathbf{P}_{D} / I_{\Lambda}(D)$. Учитьвая, что преобразование Лапласа отождествляет пространства $\mathbf{P}_{D} / I_{W}(D)$ и $H^{*}(D) /$ ort $W$, получаем следуюшие изоморфизмы:

$$
W \cong\left[H^{*}(D) / \text { ort } W\right]^{*} \cong\left[\mathbf{P}_{D} / I_{\Lambda}(D)\right]^{*}
$$

Пусть $\varphi$ - произвольная функция из $W$ и функционалы $s \in\left[H^{*}(D) / \operatorname{ort} W\right]^{*}$ и $\mu \in\left[\mathbf{P}_{D} / I_{\Lambda}(D)\right]^{*}$ сопоставляются ей при указанных изоморфизмах. Фиксируем $z \in D$. Если $\delta_{z}-$ функционал Дирака, сосредоточенный в точке $z$, то

$$
\varphi(z)=\left(\delta_{z}, \varphi\right)=\left(\left[\delta_{z}\right], \varphi\right)=\left(s,\left[\delta_{z}\right]\right)=\left(\mu,\left[f_{z}\right]\right)
$$

где $f_{z}$ - преобразование Лапласа функционала $\delta_{z}$. Функция $f_{z}(\eta)$, как легко видеть, совпадает с $\exp (z \eta)$. Таким образом, имеет место равенство $\varphi(z)=$ $(\mu,[\exp (z \eta)])$. Согласно утверждению 1$)$ пространство $\mathbf{P}_{D} / I_{\Lambda}(D)$ изоморфно $\Phi$. Поэтому найдется функционал $\tau \in \Phi^{*}$ такой, что

$$
\varphi(z)=(\mu,[\exp (z \eta)])=(\tau, \Sigma[\exp (z \eta)])=\left(\tau,\left\{z^{n-1} \exp \left(\lambda_{k} z\right)\right\}\right)
$$


Выберем номер $m$ такой, что $b=\left\{b_{k, n}\right\}=\left\{z^{n-1} \exp \left(\lambda_{k} z\right)\right\} \in \Phi_{m}$. Функционал $\tau$ непрерывен на $\Phi^{*}$. Поэтому его сужение на банахово пространство $\Phi_{m+1}$ (как и на любое другое $\Phi_{s}$ ) будет непрерывным (см. [49, гл. $5, \S 2$, предложение 5]), т. е. $|(\tau, \tilde{b})| \leqslant c\|\tilde{b}\|_{m+1} \forall \tilde{b} \in \Phi_{m+1}$. Пусть $d_{k, n}$ равно значению функционала $\tau$ на элементе $e^{k, n}=\left\{b_{l, s}^{\prime}\right\}$, где $b_{l, s}^{\prime}$ равен единице, когда $l=k, s=n$, и равен нулю для всех остальных значений индексов $l, s$. Тогда из предыдушего с учетом неравенства (1.1) получаем

$$
\begin{aligned}
& \left|\varphi(z)-\sum_{\substack{k=1, \ldots, j-1 \\
n=1, \ldots, m_{k}}} d_{k, n} b_{k, n}-\sum_{n=1}^{p} d_{j, n} b_{j, n}\right| \\
& =\left|(\tau, b)-\sum_{\substack{k=1, \ldots, j-1 \\
n=1, \ldots, m_{k}}} d_{k, n} b_{k, n}-\sum_{n=1}^{p} d_{j, n} b_{j, n}\right| \\
& =\left|(\tau, b)-\left(\tau,\left(b_{1,1}, \ldots, b_{1, m_{1}}, b_{2,1}, \ldots, b_{j-1, m_{j-1}}, b_{j, 1}, \ldots, b_{j, p}, 0, \ldots\right)\right)\right| \\
& \leqslant c \sup _{k>j-1, n=1, \ldots, m_{k}}\left|b_{k, n}\right| \exp \left(-H_{K_{m}}\left(\lambda_{k}\right)\right) \\
& =c \sup _{k>j-1, n=1, \ldots, m_{k}}\left|b_{k, n}\right| \exp \left(-H_{K_{m}}\left(\lambda_{k}\right)\right) \exp \left(H_{K_{m}}\left(\lambda_{k}\right)-H_{K_{m+1}}\left(\lambda_{k}\right)\right) \\
& \leqslant c\|b\|_{m} \sup _{k>j-1, n=1, \ldots, m_{k}} \exp \left(H_{K_{m}}\left(\lambda_{k}\right)-H_{K_{m+1}}\left(\lambda_{k}\right)\right) \\
& \leqslant c\|b\|_{m} \sup _{k>j-1, n=1, \ldots, m_{k}} \exp \left(-\alpha_{m}\left|\lambda_{k}\right|\right) \rightarrow 0, \quad j \rightarrow \infty .
\end{aligned}
$$

Таким образом, $\varphi(z)=\sum_{k, n} d_{k, n} z^{n-1} \exp \left(\lambda_{k} z\right)$. Предложение доказано.

\section{§3. Достаточные условия интерполяции}

Прежде чем перейти к формулировке и доказательству основного результата этого параграфа, введем обозначения и докажем некоторые вспомогательные утверждения. Для последовательности $\Lambda=\left\{\lambda_{k}, m_{k}\right\}$, где $\left|\lambda_{k}\right| \rightarrow \infty$ (если она бесконечна), введем функции

$$
q_{\Lambda}(z, y, \delta)=\prod_{\lambda_{k} \in B(y, \delta|y|)}\left(\frac{z-\lambda_{k}}{3 \delta\left|\lambda_{k}\right|}\right)^{m_{k}}, \quad q_{\Lambda}^{j}(z, y, \delta)=q_{\Lambda}(z, y, \delta)\left(\frac{z-\lambda_{j}}{3 \delta\left|\lambda_{j}\right|}\right)^{-m_{j}}
$$

причем вторая функция определяется лишь для тех $j$, для которых $\lambda_{j}$ принадлежит $B(y, \delta|y|)$. Модуль функции $q_{\Lambda}(z, y, \delta)$ можно интерпретировать как меру сгущения точек $\lambda_{k} \in B(y, \delta|y|)$ около $z$. В случае, когда $B(y, \delta|y|)$ не содержит ни одной из $\lambda_{k}$, считаем, что $q_{\Lambda}(z, y, \delta) \equiv 1$ и $q_{\Lambda}^{j}(z, y, \delta) \equiv 1$. Отметим, что модуль каждого из сомножителей в определении $q_{\Lambda}^{j}(z, y, \delta)$ и $q_{\Lambda}(z, y, \delta)$ в круге $B(y, \delta|y|)$ оценивается сверху величиной $2(3(1-\delta))^{-1}$ (для $\left.\delta \in(0,1)\right)$, т. е. при $\delta \in(0,1 / 3)$ он не превосходит единицы. Кроме того, если $\delta_{1} \leqslant \delta_{2}$ и $B\left(y_{1}, \delta_{1}\left|y_{1}\right|\right) \subseteq B\left(y_{2}, \delta_{2}\left|y_{2}\right|\right)$, то число сомножителей в определении $q_{\Lambda}^{j}\left(z, y_{1}, \delta_{1}\right)$ не превосходит числа сомножителей в определении $q_{\Lambda}^{j}\left(z, y_{2}, \delta_{2}\right)$ и каждый из сомножителей для $q_{\Lambda}^{j}\left(z, y_{1}, \delta_{1}\right)$ по 
модулю не меньше соответствуюшего сомножителя для $q_{\Lambda}^{j}\left(z, y_{2}, \delta_{2}\right)$. Таким образом, имеем

$$
\left|q_{\Lambda}^{j}\left(z, y_{1}, \delta_{1}\right)\right| \geqslant\left|q_{\Lambda}^{j}\left(z, y_{2}, \delta_{2}\right)\right|, \quad z \in B\left(y_{2}, \delta_{2}\left|y_{2}\right|\right),
$$

если $0<\delta_{1} \leqslant \delta_{2}<1 / 3$ и $B\left(y_{1}, \delta_{1}\left|y_{1}\right|\right) \subseteq B\left(y_{2}, \delta_{2}\left|y_{2}\right|\right)$.

Аналогичное неравенство верно и для $q_{\Lambda}(z, y, \delta)$ :

$$
\left|q_{\Lambda}\left(z, y_{1}, \delta_{1}\right)\right| \geqslant\left|q_{\Lambda}\left(z, y_{2}, \delta_{2}\right)\right|, \quad z \in B\left(y_{2}, \delta_{2}\left|y_{2}\right|\right),
$$

если $0<\delta_{1} \leqslant \delta_{2}<1 / 3, B\left(y_{1}, \delta_{1}\left|y_{1}\right|\right) \subseteq B\left(y_{2}, \delta_{2}\left|y_{2}\right|\right)$. Положим $S_{\Lambda}=0$, если $\Lambda$ состоит из конечного числа элементов, и

$$
S_{\Lambda}=\lim _{\delta \rightarrow 0} \underset{k \rightarrow \infty}{\lim _{k \rightarrow \infty}}\left|\lambda_{k}\right|^{-1} \ln \left|q_{\Lambda}^{k}\left(\lambda_{k}, \lambda_{k}, \delta\right)\right|
$$

в противном случае. Это определение корректно, поскольку в силу (3.1) предел по $\delta$ всегда существует. В силу сказанного выше $S_{\Lambda} \leqslant 0$. Положим

$$
M_{\Lambda}(y, \delta)=\sum_{\lambda_{k} \in B(y, \delta|y|)} m_{k}, \quad M_{\Lambda}=\lim _{\delta \rightarrow 0} \varlimsup_{k \rightarrow \infty}\left|\lambda_{k}\right|^{-1} M_{\Lambda}\left(\lambda_{k}, \delta\right),
$$

причем считаем $M_{\Lambda}=0$, если $\Lambda$ конечна. Очевидно, что $M_{\Lambda} \geqslant m(\Lambda) \geqslant 0$ и

$$
M_{\Lambda}\left(y_{1}, \delta_{1}\right) \leqslant M_{\Lambda}\left(y_{1}, \delta_{2}\right), \quad B\left(y_{1}, \delta_{1}\left|y_{1}\right|\right) \subset B\left(y_{2}, \delta_{2}\left|y_{2}\right|\right) .
$$

Отсюда следует, что определение $M_{\Lambda}$ также корректно. Отметим еще, что если некоторая целая функция экспоненциального типа обращается в нуль (по крайней мере) в точках $\lambda_{k}$ с кратностью, не меньшей, чем $m_{k}$, то по тем же соображениям, что и для $m(\Lambda)$, величина $M_{\Lambda}$ конечна.

Лемма 3.1. Пусть последовательность $\Lambda$ такова, что $S_{\Lambda} \geqslant a>-\infty u$ $M_{\Lambda} \leqslant A<+\infty$. Тогда для каждого $\varepsilon>0$ найдется $\delta_{0}>0$ такое, что для всех $\delta \in\left(0, \delta_{0}\right)$ выполнено неравенство

$$
\left|q_{\Lambda}\left(y, \lambda_{k}, \delta\right)\right| \geqslant \widetilde{C}_{1} d_{k} \quad \forall y: 2^{-1} \rho_{k}(\delta)-\widetilde{C}_{2} d_{k} \leqslant\left|y-\lambda_{k}\right| \leqslant 2^{-1} \rho_{k}(\delta),
$$

где $d_{k}=\exp \left((2 a-3 A-\varepsilon)\left|\lambda_{k}\right|\right), \quad \rho_{k}(\delta)=\min \left(\rho_{k}, \delta\left|\lambda_{k}\right|\right), \quad \rho_{k}=\min _{j \neq k}\left|\lambda_{k}-\lambda_{j}\right|$, $k=1,2, \ldots$, и постоянные $\widetilde{C}_{1}, \widetilde{C}_{2}>0$ зависят лишь от $\delta$.

ДокАЗАТЕЛЬСТво. Пусть $0<\tilde{\varepsilon}<\varepsilon / 8$. Согласно определению величины $S_{\Lambda}$ найдем $\delta_{0} \in(0,1 / 6)$ такое, что $\underline{\lim }_{j \rightarrow \infty}\left|\lambda_{j}\right|^{-1} \ln \left|q_{\Lambda}^{j}\left(\lambda_{j}, \lambda_{j}, 2 \delta_{0}\right)\right| \geqslant a-\tilde{\varepsilon}$. Выберем теперь номер $j_{0}$ так, чтобы $\left|\lambda_{j}\right|^{-1} \ln \left|q_{\Lambda}^{j}\left(\lambda_{j}, \lambda_{j}, 2 \delta_{0}\right)\right| \geqslant a-2 \tilde{\varepsilon}, j \geqslant j_{0}$. Тогда с учетом (3.1) имеем

$$
\left|q_{\Lambda}^{j}\left(\lambda_{j}, \lambda_{j}, \delta\right)\right| \geqslant \exp \left((a-2 \tilde{\varepsilon})\left|\lambda_{j}\right|\right), \quad j \geqslant j_{0}, \quad \delta \in\left(0,2 \delta_{0}\right) .
$$

$\mathrm{B}$ силу определения величины $M_{\Lambda}$, уменьшая при необходимости $\delta_{0}>0$ и увеличивая $j_{0}$, можно считать, что

$$
M_{\Lambda}\left(\lambda_{j}, 2 \delta_{0}\right) \leqslant(A+2 \tilde{\varepsilon})\left|\lambda_{j}\right|, \quad j \geqslant j_{0} .
$$


Отсюда и из (3.3) получаем

$$
M_{\Lambda}\left(\lambda_{j}, \delta\right) \leqslant(A+2 \tilde{\varepsilon})\left|\lambda_{j}\right|, \quad j \geqslant j_{0}, \quad \delta \in\left(0,2 \delta_{0}\right) .
$$

Пусть $j \geqslant j_{0}, \delta \in\left(0,2 \delta_{0}\right)$ и $\rho_{j}(\delta)=\rho_{j}$. Рассмотрим произвольное $z$ такое, что $\left|z-\lambda_{j}\right|=\rho_{j} / 2$. Для всех $k=1,2, \ldots$ справедливы соотношения

$$
\left|z-\lambda_{k}\right| \geqslant\left|\lambda_{j}-\lambda_{k}\right|-\left|z-\lambda_{j}\right|=\left|\lambda_{j}-\lambda_{k}\right|-\rho_{j} / 2 \geqslant\left|\lambda_{j}-\lambda_{k}\right| / 2 .
$$

Тогда с учетом $(3.4),(3.5)$ и неотрицательности числа $b$ имеем

$$
\begin{aligned}
\left|q_{\Lambda}^{j}\left(z, \lambda_{j}, \delta\right)\right| & \geqslant \exp \left[\ln (1 / 2)(A+2 \tilde{\varepsilon})\left|\lambda_{j}\right|\right]\left|q_{\Lambda}^{j}\left(\lambda_{j}, \lambda_{j}, \delta\right)\right| \\
& \geqslant \exp \left[(\ln (1 / 2)(A+2 \tilde{\varepsilon})+a-2 \tilde{\varepsilon})\left|\lambda_{j}\right|\right] \geqslant \exp \left[(a-A-4 \tilde{\varepsilon})\left|\lambda_{j}\right|\right]
\end{aligned}
$$

для любого $z$ такого, что

$$
\left|z-\lambda_{j}\right|=\rho_{j} / 2=\rho_{j}(\delta) / 2 .
$$

Пусть теперь $\rho_{j}(\delta)=\delta\left|\lambda_{j}\right|$. Тогда в силу определений величины $\rho_{j}(\delta)$ и функции $q_{\Lambda}^{j}$ с учетом неравенств $a \leqslant 0$ и $A \geqslant 0$ получаем

$$
1 \equiv\left|q_{\Lambda}^{j}\left(z, \lambda_{j}, \delta\right)\right| \geqslant \exp \left((a-A-4 \tilde{\varepsilon})\left|\lambda_{j}\right|\right) \quad \forall z:\left|z-\lambda_{j}\right|=\rho_{j}(\delta) / 2 .
$$

Отсюда и из (3.6) имеем оценку

$$
\left|q_{\Lambda}^{j}\left(z, \lambda_{j}, \delta\right)\right| \geqslant \exp \left((a-A-4 \tilde{\varepsilon})\left|\lambda_{j}\right|\right)
$$

для любого $z$ такого, что

$$
\left|z-\lambda_{j}\right|=\rho_{j}(\delta) / 2, \quad j \geqslant j_{0}, \quad \delta \in\left(0,2 \delta_{0}\right) .
$$

Выберем $j_{1} \geqslant j_{0}$ так, что для любого $j \geqslant j_{1}$ каждый номер $k$, для которого $\lambda_{k} \in B\left(\lambda_{j}, \delta_{0}\left|\lambda_{j}\right|\right)$, будет не меньше $j_{0}$. Пусть $j \geqslant j_{1}, \delta \in\left(0, \delta_{0}\right), \rho_{j}(\delta)=\rho_{j}$ и $k_{j}$ - один из номеров таких, что $\left|\lambda_{j}-\lambda_{k_{j}}\right|=\rho_{j}$. Поскольку $\rho_{j} \leqslant \delta\left|\lambda_{j}\right|$, то $\left|\lambda_{k_{j}}\right| \geqslant(1-\delta)\left|\lambda_{j}\right|$. Следовательно, с учетом неравенства $\delta<1 / 2$ имеем

$$
\left|\lambda_{j}-\lambda_{k_{j}}\right| \leqslant \delta\left|\lambda_{j}\right| \leqslant \delta(1-\delta)^{-1}\left|\lambda_{k_{j}}\right|<2 \delta\left|\lambda_{k_{j}}\right|
$$

т. е. $\lambda_{j} \in B\left(\lambda_{k_{j}}, 2 \delta\left|\lambda_{k_{j}}\right|\right)$. Так как для наших $\delta$ множители в произведении, определяющем функцию $q_{\Lambda}^{k_{j}}\left(z, \lambda_{k_{j}}, 2 \delta\right)$, не превосходят по модулю единицы, то в силу (3.4) получаем

$$
\left|\lambda_{j}-\lambda_{k_{j}}\right|^{m_{j}}\left(6 \delta\left|\lambda_{j}\right|\right)^{-m_{j}} \geqslant\left|q_{\Lambda}^{k_{j}}\left(\lambda_{k_{j}}, \lambda_{k_{j}}, 2 \delta\right)\right| \geqslant \exp \left[(a-2 \tilde{\varepsilon})\left|\lambda_{j}\right|\right] .
$$

Отсюда с учетом неотрищательности $A$ имеем

$$
\left|\lambda_{j}-z\right|^{m_{j}}\left(3 \delta\left|\lambda_{j}\right|\right)^{-m_{j}}=\left|\lambda_{j}-\lambda_{k_{j}}\right|^{m_{j}}\left(6 \delta\left|\lambda_{j}\right|\right)^{-m_{j}} \geqslant \exp \left[(a-2 A-4 \tilde{\varepsilon})\left|\lambda_{j}\right|\right]
$$


для любого z такого, что

$$
\left|z-\lambda_{j}\right|=\frac{\rho_{j}(\delta)}{2}=\frac{\rho_{j}}{2}=\frac{\left|\lambda_{j}-\lambda_{k_{j}}\right|}{2} .
$$

Пусть теперь $\rho_{j}(\delta)=\delta\left|\lambda_{j}\right|$. Тогда с учетом (3.5) и того, что $a \leqslant 0$, получаем

$$
\begin{gathered}
\left|\lambda_{j}-z\right|^{m_{j}}\left(3 \delta\left|\lambda_{j}\right|\right)^{-m_{j}}=\left(\delta\left|\lambda_{j}\right|\right)^{m_{j}}\left(6 \delta\left|\lambda_{j}\right|\right)^{-m_{j}} \geqslant \exp \left(\ln (1 / 6)(A+2 \tilde{\varepsilon})\left|\lambda_{j}\right|\right) \\
\geqslant \exp \left((a-2 A-4 \tilde{\varepsilon})\left|\lambda_{j}\right|\right) \quad \forall z:\left|z-\lambda_{j}\right|=\frac{\rho_{j}(\delta)}{2}=\frac{\delta\left|\lambda_{j}\right|}{2} .
\end{gathered}
$$

Отсюда, из $(3.7),(3.8)$ и определения функции $q_{\Lambda}\left(z, \lambda_{j}, \delta\right)$ следует, что

$$
\left|q_{\Lambda}\left(z, \lambda_{j}, \delta\right)\right| \geqslant \exp \left[(2 a-3 A-8 \tilde{\varepsilon})\left|\lambda_{j}\right|\right]
$$

для любого z такого, что

$$
\left|z-\lambda_{j}\right|=\frac{\rho_{j}(\delta)}{2}, \quad j \geqslant j_{1}, \quad \delta \in\left(0, \delta_{0}\right) .
$$

Пусть $\delta \in\left(0, \delta_{0}\right)$. Выберем $j_{0}(\delta) \geqslant j_{1}$ так, что $\delta\left|\lambda_{j}\right| \geqslant 2$ для всех $j \geqslant j_{0}(\delta)$. Тогда круг $B(z, 1)$ лежит в $B\left(\lambda_{j}, \delta\left|\lambda_{j}\right|\right)$ для всех $z \in B\left(\lambda_{j}, \delta\left|\lambda_{j}\right| / 2\right)$ и $j \geqslant j_{0}(\delta)$. Поскольку функция $q_{\Lambda}\left(y, \lambda_{j}, \delta\right)$ не превосходит по модулю единицы в $B\left(\lambda_{j}, \delta\left|\lambda_{j}\right|\right)$, то из интегральной формулы Коши следует, что этим же свойством, но уже в круге $B\left(\lambda_{j}, \delta\left|\lambda_{j}\right| / 2\right)$, обладает и ее производная. Используя теперь формулу для первообразной, получаем оценку

$$
\left|q_{\Lambda}\left(y, \lambda_{j}, \delta\right)-q_{\Lambda}\left(z, \lambda_{j}, \delta\right)\right| \leqslant|y-z|, \quad y, z \in \overline{B\left(\lambda_{j}, \delta\left|\lambda_{j}\right| / 2\right)} .
$$

Отсюда с учетом (3.9) и выбора $\tilde{\varepsilon}$ находим, что

$$
\left|q_{\Lambda}\left(y, \lambda_{j}, \delta\right)\right| \geqslant 2^{-1} \exp \left[(2 a-3 A-\varepsilon)\left|\lambda_{j}\right|\right], \quad j \geqslant j_{0}(\delta)
$$

для любого $y$ такого, что

$$
2^{-1}\left(\rho_{j}(\delta)-\exp \left[(2 a-3 A-\varepsilon)\left|\lambda_{j}\right|\right]\right) \leqslant\left|y-\lambda_{j}\right| \leqslant 2^{-1} \rho_{j}(\delta), \quad \delta \in\left(0, \delta_{0}\right) .
$$

Выбирая подходяшие постоянные $\widetilde{C}_{1}, \widetilde{C}_{2}>0$ в зависимости лишь от номера $j_{0}(\delta)$ (а значит, лишь от $\delta$ ), получаем требуемое утверждение.

Докажем теперь некоторые вспомогательные утверждения относительно целых функций экспоненциального типа. Из первого утверждения получим оценку числа нулей таких функций в круге $B(y, \delta|y|)$.

ЛЕмма 3.2. Пусть $\Lambda=\left\{\lambda_{k}, m_{k}\right\} ; \varphi-$ челая функция әкспоненциального типа, обращающаяся в нуль (по крайней мере) в точках $\lambda_{k}$ с кратностью, не меньшей, чем $m_{k} ; H(z)$ - положительно однородная порядка один функиия; $\tau \in(0,1)$ и $F-$ подмножество $\mathbf{S}$ такие, что

$$
h_{\varphi}(\zeta) \leqslant H(w), \quad \zeta \in B(w, \tau), \quad w \in F
$$

Тогда верно неравенство

$$
\varlimsup_{\substack{|y| \rightarrow \infty \\ y /|y| \in F, \varphi(y) \neq 0}}|y|^{-1}\left(M_{\Lambda}(y, \delta)-H(y)+\ln |\varphi(y)|\right) \leqslant 0, \quad \delta \in(0, \tau / 3) .
$$


ДоКАЗАТЕЛЬСТВО. Фиксируем произвольное число $\tilde{\varepsilon}>0$. По свойству индикаторов (см., например, $\left[1\right.$, гл. $1, \S 6$, свойство г)]) найдем $T_{1}>0$ такое, что

$$
\ln |\varphi(z)| \leqslant h_{\varphi}(z)+\tilde{\varepsilon}|z| \quad \forall z:|z| \geqslant T_{1} .
$$

В силу (3.10) верно неравенство

$$
h_{\varphi}(z)+\tilde{\varepsilon}|z| \leqslant H(y)+\tilde{\varepsilon}(1+\tau)|y| \leqslant H(y)+2 \tilde{\varepsilon}|y|, \quad z \in B(y, \tau|y|), \quad y /|y| \in F .
$$

Пусть $\varphi(y) \neq 0, y /|y| \in F,|y| \geqslant(1-\tau)^{-1} T_{1}$. Тогда в круге $B(y, \tau|y|)$ выполнено (3.11). Следовательно,

$$
\ln |\varphi(z)| \leqslant H(y)+2 \tilde{\varepsilon}|y|, \quad z \in B(y, \tau|y|) .
$$

Пусть $\delta \in(0, \tau / 3)$ и $m=M_{\Lambda}(y, \delta)$. Из последней оценки и теоремы о нулях аналитической функции в круге (см., например, $[48$, гл. $1, \S 2$, теорема 2.1]) легко следует, что

$$
e^{m} \leqslant \exp (H(y)-\ln |\varphi(y)|+2 \tilde{\varepsilon}|y|) .
$$

В силу произвольности выбора $\tilde{\varepsilon}>0$ отсюда получаем требуемое неравенство.

В следующих двух утверждениях будут получены другие оценки целых функций экспоненциального типа. Но прежде сделаем одно простое замечание, которым будем неоднократно пользоваться в дальнейшем. Пусть функция $h_{0}(z)$ вещественнозначна и положительно однородна порядка один, $F \subset \mathbf{S}, \varepsilon>0$ и $\tau \in(0,1 / 3)$ такие, что

$$
\left|h_{0}(y)-h_{0}(w)\right| \leqslant \varepsilon / 3 \quad \forall w \in F, \quad y \in B(w, \tau) .
$$

Тогда верно неравенство

$$
\sup _{y \in B(z, \tau|z|)} h_{0}(y) \leqslant \inf _{y \in B(z, \tau|z|)} h_{0}(y)+\varepsilon \inf _{y \in B(z, \tau|z|)}|y| \quad \forall z: z /|z| \in F
$$

Действительно, в силу положительной однородности функции $h_{0}(y)$ имеем

$$
\begin{aligned}
& \left|h_{0}(y)-h_{0}(x)\right| \leqslant\left|h_{0}(y)-h_{0}(z)\right|+\left|h_{0}(x)-h_{0}(z)\right| \leqslant|z|\left|h_{0}(y /|z|)-h_{0}(z /|z|)\right| \\
& \quad+|z|\left|h_{0}(x /|z|)-h_{0}(z /|z|)\right| \leqslant 3^{-1} 2 \varepsilon|z| \quad \forall z: z /|z| \in F, \quad y, x \in B(z, \tau|z|) .
\end{aligned}
$$

Отсюда следует, что

$$
\sup _{y \in B(z, \tau|z|)} h_{0}(y) \leqslant \inf _{y \in B(z, \tau|z|)} h_{0}(y)+3^{-1} 2 \varepsilon|z| \quad \forall z: z /|z| \in F .
$$

Заметим, что $|z|=(1-\tau)^{-1} \inf _{y \in B(z, \tau|z|)}|y| \forall z \neq 0$. Поэтому, учитывая включение $\tau \in(0,1 / 3)$, из предыдущего легко получаем неравенство (3.13).

Для множества $R \subset \mathbb{C}$ через $R^{\delta}, \delta>0$, обозначим объединение кругов $\cup B(z, \delta|z|)$, где $z$ принимает значения из $R$. 
Лемма 3.3. Пусть $\varphi-$ иелая функиия экспоненииального типа, функиии $h(z), H(z)$ непрерывны и положстельно однородны порядка один в $\mathbb{C}, \alpha, \varepsilon>0$ $u R$ - неограниченное множество такие, что

$$
h_{\varphi}(z) \leqslant H(z), \quad z \in R^{\alpha}, \quad \underset{z \in R,|z| \rightarrow \infty}{\lim }|z|^{-1}(\ln |\varphi(z)|-h(z)) \geqslant 0 .
$$

Тогда существуют постоянная $\delta_{0}>0$ (зависящая только от $h, H, \alpha u \varepsilon$ ) u $T>0$ такие, что для каждого $\delta \in\left(0, \delta_{0}\right)$ найдется набор открытых, попарно непересекающихся множеств $U_{p}, \quad p=1,2, \ldots$, обладающих следующими свойствами:

1) для каждого $p \geqslant 1$ и любого $y \in U_{p}$ имеет место вложение $U_{p} \subset$ $B(y, 88 \delta|y|)$;

2) $U_{p} \subset R^{22 \delta}, p \geqslant 1$

3) $R^{\delta} \backslash B(0, T) \subset \cup \bar{U}_{p}$;

4) для каждого $p \geqslant 1$ выполнено неравенство

$$
\ln |\varphi(z)| \geqslant h(z)-2 \varepsilon|z|-(3+\ln 24)(H(z)-h(z)+3 \varepsilon|z|), \quad z \in \partial U_{p}
$$

ДоКАЗАТЕЛЬСТво. По условию найдем $T_{1}>1$ такое, что

$$
|z|^{-1}(\ln |\varphi(z)|-h(z)) \geqslant-\varepsilon \quad \forall z \in R \backslash B\left(0, T_{1}\right)
$$

Поскольку функции $H(z)+\varepsilon|z|$ и $h(z)-\varepsilon|z|-(3+\ln 24)(H(z)-h(z)+3 \varepsilon|z|)$ непрерывны (а поэтому и равномерно непрерывны на $\mathbf{S}$ ), то для некоторого $\tau \in(0,1 / 3)$, $\tau<\alpha$, и $F=\mathbf{S}$ будет выполнено (3.12), а значит, и (3.13), если в качестве $h_{0}$ взять одну из них. Положим $\delta_{0}=\tau / 132$ и фиксируем $\delta \in\left(0, \delta_{0}\right)$. Отметим, что $\delta_{0}$ зависит только от $\tau$, которое, в свою очередь, зависит лишь от $h, H, \alpha$ и $\varepsilon$, но не зависит от $\varphi$ и $R$. На окружности $\mathbf{S}$ выберем точки $x_{1}, \ldots, x_{m}$ так, что объединение кругов $B\left(x_{j}, \delta\right), j=\overline{1, m}$, покрывает ее. Для всех $j=\overline{1, m}$ положим $x_{j, 1}=x_{j}$ и $x_{j, s}=(1+\delta) x_{j, s-1}, s \geqslant 2$. Легко видеть, что круги $B\left(x_{j, s}, 2 \delta\left|x_{j, s}\right|\right)$, $j=\overline{1, m}, s \geqslant 1$, покрывают в совокупности внешность единичного круга с центром в нуле. Из набора точек $x_{j, s}, j=\overline{1, m}, s \geqslant 1$, удалим все те, для которых круг $B\left(x_{j, s}, 2 \delta\left|x_{j, s}\right|\right)$ не пересекает $R \backslash B\left(0, T_{1}\right)$. Оставшиеся точки перенумеруем в произвольном порядке и обозначим $w_{1}, w_{2}, \ldots$ По построению

$$
R \backslash B\left(0, T_{1}\right) \subset \cup B\left(w_{p}, 2 \delta\left|w_{p}\right|\right) .
$$

Пусть $z_{p}, y \in\left(R \backslash B\left(0, T_{1}\right)\right) \cap B\left(w_{p}, 2 \delta\left|w_{p}\right|\right)$. Тогда круг $B\left(z_{p}, 11 \delta\left|z_{p}\right|\right)$ содержит любое $z \in B(y, \delta|y|)$. Действительно, с учетом неравенства $\delta<1 / 4$ имеем

$$
\begin{aligned}
\left|z_{p}-z\right| & \leqslant\left|z_{p}-w_{p}\right|+\left|w_{p}-y\right|+|y-z| \leqslant 2 \delta\left|w_{p}\right|+2 \delta\left|w_{p}\right|+\delta|y| \\
& \leqslant 4 \delta\left|w_{p}\right|+(1+2 \delta) \delta\left|w_{p}\right| \leqslant \frac{11 \delta\left|w_{p}\right|}{2} \leqslant \frac{11 \delta\left|z_{p}\right|}{2(1-2 \delta)} \leqslant 11 \delta\left|z_{p}\right| .
\end{aligned}
$$

Положим $T \geqslant 2 T_{1}>(1+\delta) T_{1}$, и пусть $z \in R^{\delta} \backslash B(0, T)$. Тогда $z \in B(y, \delta|y|)$ для некоторой точки $y \in R \backslash B\left(0, T_{1}\right)$, которая лежит в одном из кругов $B\left(w_{p}, 2 \delta\left|w_{p}\right|\right)$. 
Следовательно, по доказанному $z \in B\left(z_{p}, 11 \delta\left|z_{p}\right|\right)$. Таким образом, объединение $\bigcup_{p} B\left(z_{p}, 11 \delta\left|z_{p}\right|\right)$ содержит множество $R^{\delta} \backslash B(0, T)$.

Увеличивая при необходимости $T_{1}>0$, можно считать, что для всех $p \geqslant 1$ в $B\left(z_{p}, 132 \delta\left|z_{p}\right|\right)$ выполнено (3.11) для $\tilde{\varepsilon}=\varepsilon$. Тогда по условию леммы имеем

$$
\ln |\varphi(z)| \leqslant H(z)+\varepsilon|z| \quad \forall z \in R^{\alpha}, \quad|z| \geqslant T_{1} .
$$

Отсюда с учетом (3.13) имеем

$$
\ln |\varphi(z)| \leqslant H\left(z_{p}\right)+2 \varepsilon\left|z_{p}\right|, \quad z \in B\left(z_{p}, 132 \delta\left|z_{p}\right|\right), \quad p=1,2, \ldots
$$

Рассмотрим функцию $\varphi_{p}(z)=\varphi(z) / \varphi\left(z_{p}\right)$. Она обладает следующими свойствами: $\varphi_{p}\left(z_{p}\right)=1$, а в силу (3.14) и (3.15)

$$
\ln \left|\varphi_{p}(z)\right| \leqslant H\left(z_{p}\right)-h\left(z_{p}\right)+3 \varepsilon\left|z_{p}\right|, \quad z \in B\left(z_{p}, 132 \delta\left|z_{p}\right|\right), \quad p=1,2, \ldots
$$

Тогда по теореме об оценке снизу модуля аналитической функции (см., например, $[48$, гл. $1, \S 4$, теорема 4.2]) имеет место неравенство

$$
\ln \left|\varphi_{p}(z)\right| \geqslant-(3+\ln 24)\left(H\left(z_{p}\right)-h\left(z_{p}\right)+3 \varepsilon\left|z_{p}\right|\right),
$$

которое выполнено в $B\left(z_{p}, 22 \delta\left|z_{p}\right|\right)$, но вне исключительных кружков с суммой радиусов, равной $11 \delta\left|z_{p}\right| / 2$. Отсюда с учетом (3.14) и (3.13) получаем

$$
\ln |\varphi(z)| \geqslant h(z)-2 \varepsilon|z|-(3+\ln 24)(H(z)-h(z)+3 \varepsilon|z|) .
$$

Поскольку сумма диаметров исключительных кружков не превосходит $11 \delta\left|z_{p}\right|$, то найдется $d_{p} \in[1,2)$ такое, что на окружности $\partial B\left(z_{p}, 11 d_{p} \delta\left|z_{p}\right|\right)$ выполнено $(3.16)$. Положим

$$
U_{1}=B\left(z_{1}, 11 d_{1} \delta\left|z_{1}\right|\right), \quad U_{p}=B\left(z_{p}, 11 d_{p} \delta\left|z_{p}\right|\right) \backslash\left[\bigcup_{j=\overline{1, p-1}} \bar{U}_{j}\right], \quad p \geqslant 2 .
$$

Тогда $U_{p}, p \geqslant 1$, обладают свойствами 1$\left.)-4\right)$ из утверждения леммы. Действительно, свойство 4) следует из (3.16) и того, что граница $U_{p}$ состоит из дуг окружностей $\partial B\left(z_{j}, 11 d_{j} \delta\left|z_{j}\right|\right)$; свойство 3$)$ имеет место, поскольку $\bigcup_{p} \bar{U}_{p}$ содержит множество $\bigcup_{p} \overline{B\left(z_{p}, 11 \delta\left|z_{p}\right|\right)}$, которое, в свою очередь, содержит $R^{\delta} \backslash B(0, T)$. Наконец, свойства 2) и 1) легко следуют из вложения $U_{p} \subset B\left(z_{p}, 11 d_{p} \delta\left|z_{p}\right|\right)$ и неравенства $22 \delta<1 / 2$. Лемма доказана.

ЗАмЕЧАНИЕ 3.4. Если в условиях леммы $3.3 h(z)=c_{1}|z|, H(z)=c_{2}|z|$ и $\varepsilon=1$, то число $\delta_{0}>0$ можно выбрать так, что оно не будет зависеть от $h, H$ и $\varepsilon$ (т. е. от $\left.c_{1}, c_{2}\right)$, но при этом ухудшится оценка в свойстве 4$)$ для $U_{p}$. Действительно, пусть $0<\tau<\min \{\alpha, 1 / 2\}$. Тогда для любого $z \neq 0$

$$
\begin{array}{ll}
\sup _{y \in B(z, \tau|z|)} c|y| \leqslant 3 \inf _{y \in B(z, \tau|z|)} c|y|, & c>0, \\
\sup _{y \in B(z, \tau|z|)} c|y| \leqslant 3^{-1} \inf _{y \in B(z, \tau|z|)} c|y|, & c>0 .
\end{array}
$$

Определяя по $\tau$, как и в лемме, число $\delta_{0}$ и пользуясь этими неравенствами вместо $(3.13)$, мы точно так же для каждого $\delta \in\left(0, \delta_{0}\right)$ построим множества $U_{p}$, обладающие свойствами 1)-3) и свойством

$\left.4^{\prime}\right) \ln |\varphi(z)| \geqslant \tilde{a}|z|, z \in \partial U_{p}$, где $\tilde{a}$ зависит только от $c_{1}, c_{2}$. 
Лемма 3.5. Пусть $\varphi-$ иелая функиия экспоненииального типа, функиии $h(z), H(z)$ непрерывны и положительно однородны порядка один в $\mathbb{C}, \alpha, \varepsilon>0$ $u R$ - неограниченное множество такие, что

$$
h_{\varphi}(z) \leqslant H_{1}(z) \quad \forall z \in \widetilde{R}^{\alpha}, \quad \underline{\lim }_{z \in \widetilde{R},|z| \rightarrow \infty}|z|^{-1}\left(\ln |\varphi(z)|-h_{1}(z)\right) \geqslant 0 .
$$

Тогда существует постоянная $\widetilde{T}>0$ такая, что для $z \in \widetilde{R} u|z|>\widetilde{T}$

$$
|\varphi(y)| \geqslant \exp \left(h_{1}(y)-3 \varepsilon|y|\right), \quad y \in B\left(z, \exp \left(h_{1}(z)-H_{1}(z)-4 \varepsilon|z|\right)\right) .
$$

ДокаЗАТЕЛЬСтво. Выберем $T_{1}>0$ такое, что при $|z|>T_{1}$ выполнены (3.11) (при $\tilde{\varepsilon}=\varepsilon)$ и $(3.14)$, и $\tau \in(0,1 / 3), \tau<\alpha$, такое, что для $h_{0}(z)=H_{1}(z)+\varepsilon|z|$ и $F=\mathbf{S}$ выполнено (3.13). Можно считать, что $\tau T_{1}>2$. Пусть $z \in \widetilde{R}$ и $|z|>T_{1}+2$. Тогда в силу (3.11), условия леммы и (3.13) имеем оценку

$$
\ln |\varphi(y)| \leqslant H_{1}(z)+2 \varepsilon|z|, \quad y \in B(z, 2) .
$$

Отсюда, как и в лемме 3.1 , используя вначале интегральную формулу Коши для производной, а затем формулу для первообразной, получаем

$$
|\varphi(z)-\varphi(y)| \leqslant \exp \left(H_{1}(z)+2 \varepsilon|z|\right)|z-y|, \quad|z|>T_{1}+2, \quad y \in B(z, 1)
$$

Следовательно, с учетом (3.14) верны неравенства (не ограничивая общности, мы считаем здесь, что $\exp (-\varepsilon|z|)<1 / 2$, когда $\left.|z|>T_{1}+2\right)$

$$
\begin{aligned}
|\varphi(y)| & \geqslant|\varphi(z)|-\exp \left(H_{1}(z)+2 \varepsilon|z|\right)|z-y| \\
& \geqslant \exp \left(h_{1}(z)-\varepsilon|z|\right)-\exp \left(H_{1}(z)+2 \varepsilon|z|\right)|z-y| \\
& \geqslant \exp \left(h_{1}(z)-\varepsilon|z|\right)(1-\exp (-\varepsilon(z))) \geqslant \exp \left(h_{1}(z)-2 \varepsilon|z|\right), \\
|z| & >T_{1}+2, \quad y \in B\left(z, \exp \left(h_{1}(z)-H_{1}(z)-4 \varepsilon|z|\right)\right) .
\end{aligned}
$$

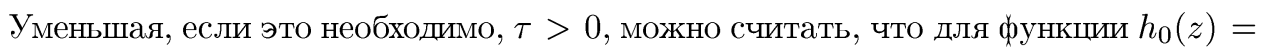
$h_{1}(z)-2 \varepsilon|z|$ выполнено (3.13). Тогда из предыдущего получаем требуемую оценку, где $\widetilde{T}=T_{1}+2$. Лемма доказана.

Прежде чем перейти к формулировке и доказательству основного результата этого параграфа, сделаем еще одно простое замечание и докажем лемму для выпуклых областей. Как мы уже отмечали, опорная функция ограниченного множества непрерывна всюду. Опорная функция неограниченного выпуклого множества $L$ непрерывна лишь во внутренности того множества, где она конечна, т. е. в $\mathbb{C} \backslash \overline{J(L)}$. Однако можно распространить понятие непрерьвности $H_{L}$ и в другие точки плоскости. Действительно, будем говорить, что $H_{L}$ непрерывна в точке $z_{0}$, если $H_{L}(z) \rightarrow H_{L}\left(z_{0}\right)$, когда $z \rightarrow z_{0}$, допуская при этом, что $H_{L}(z)$ и $H_{L}\left(z_{0}\right)$ могут принимать значение $+\infty$. В таком более широком смысле опорная функция любой неограниченной выпуклой области $D$ непрерывна всюду в плоскости, за исключением, возможно, начала координат и одного или двух лучей $\{t \xi\}$ таких, что 
$\xi \in \partial J(D) \backslash J(D)$, т. е. граничных лучей $J(D)$, на которых $H_{D}$ ограничена. Если $z_{0} \in \partial J(D) \backslash J(D)$, то

$$
\lim _{z \rightarrow z_{0}, z \in J(D)} H_{D}(z)=+\infty \neq H_{D}\left(z_{0}\right)<+\infty,
$$

но в то же время $\lim _{z \rightarrow z_{0}, z \notin J(D)} H_{D}(z)=H_{D}\left(z_{0}\right)$.

Таким образом, $H_{D}$ имеет разрыв на луче $\left\{t z_{0}, t>0\right\}$, но только если $z$ приближается к нему так, что $H_{D}(z)=+\infty$. Лучи разрьва существуют тогда и только тогда, когда граница $\partial D$ содержит точки, сколь угодно близкие к одной или двум прямым, но не имеет с ними общих точек либо содержит один или два неограниченных прямолинейных участка (лишь в двух случаях, когда $D$ - полоса или полуплоскость, эти участки являются прямыми).

Пусть $F \subset \mathbf{S}$. Через $E(\beta)$ обозначим $\beta$-окрестность $F$ на $\mathbf{S}$, т. е. $E(\beta)=\{z \in$ $\mathbf{S}|\exists \zeta \in F:| z-\zeta \mid<\beta\}$.

Лемма 3.6. Пусть $D$ - неограниченная выпуклая область, $K$ - выпуклый компакт в $D, F$ - компактное подмножество $\mathbf{S} \cap J(D)$ u $d>0$. Тогда существуют выпуклый компакт $L$ и число $\beta>0$ (в случае, когда $F \subset$ int $J(D), \quad \beta$ зависит от $F$, но не зависит от $K u d$ ) такие, что $K+L-$ компакт в $D$ и $H_{L}(z) \geqslant d|z| \quad \forall z: z /|z| \in E(\beta)$.

ДокАЗАТЕЛЬСТво. Если $D$ - полоса, то $J(D)$ - объединение двух открытых полуплоскостей. В противном случае $J(D)$ является углом (с вершиной в нуле) раствора не меньше $\pi$ (в особых случаях, когда $D$ ограничена или является полуплоскостью, $J(D)$ - соответственно вся плоскость или плоскость без луча). В любом случае $F$ можно представить как объединение двух компактов $F_{1}$ и $F_{2}$, каждый из которых лежит (в своей) полуплоскости, во внутренности которой $H_{D}$ бесконечна. Предположим, что для $F=F_{1}$ и $F=F_{2}$ мы построили соответственно компакты $L=L_{1}$ и $L=L_{2}$, которые обладают требуемыми свойствами. Тогда компакт $L$, являюшийся выпуклой оболочкой объединения $L_{1} \cup L_{2}$, будет искомым. Действительно, из вложений $K+L_{1} \subset D$ и $K+L_{2} \subset D$ следует, что $K+L \subset D$. Кроме того,

$$
H_{L}(z)=\max \left\{H_{L_{1}}(z), H_{L_{2}}(z)\right\} .
$$

Отсюда получаем требуемую оценку для $H_{L}$. Таким образом, можно считать, что для некоторого $\alpha_{0} \in \mathbb{R}$ имеет место вложение $F \subset \bar{\Pi}$, где

$$
\Pi=\left\{t \exp (i \alpha): \alpha \in\left(\alpha_{0}-\pi / 2, \alpha_{0}+\pi / 2\right), t>0\right\},
$$

и $H_{D}(z)=+\infty, z \in \Pi$. Пусть $F=F_{1} \cup F_{2}$ и $F_{1}$ лежит в той части полуплоскости $\bar{\Pi}$, где $\alpha \geqslant \alpha_{0}$, а $F_{2}-$ в той части $\bar{\Pi}$, где $\alpha \leqslant \alpha_{0}$. Считаем, что $F_{1} \neq \varnothing$, и положим $\tau_{0}=$ $\max \left\{\tau: \exp \left(i\left(\alpha_{0}+\tau\right)\right) \in F_{1}\right\}$. Пусть вначале $\tau_{0}<\pi / 2$. Тогда $F_{1}-$ компакт в П. Выберем $\beta_{1}$ так, что окрестность $E_{1}\left(\beta_{1}\right)$ множества $F_{1}$ компактно вложена в П. Поскольку $\operatorname{Re}\left(z \exp \left(-i \alpha_{0}\right)\right)>0$ для всех $z \in \Pi$, то найдется $t_{1}>0$ такое, что $t_{1} \operatorname{Re}\left(z \exp \left(-i \alpha_{0}\right)\right)>d$ для всех $z \in E_{1}\left(\beta_{1}\right)$. Если $L_{1}-$ компакт, состоящий из одной точки $t_{1} \exp \left(-i \alpha_{0}\right)$, то

$$
H_{L_{1}}(z)=t_{1} \operatorname{Re}\left(z \exp \left(-i \alpha_{0}\right)\right)>d, \quad z \in E_{1}\left(\beta_{1}\right) .
$$


Кроме того, $H_{K+L_{1}}(z)<H_{D}(z)=+\infty, z \in \Pi$, а с учетом вложения $K \subset D$ и неравенства $\operatorname{Re}\left(z \exp \left(-i \alpha_{0}\right)\right) \leqslant 0, z \notin \Pi$, имеем

$$
H_{K+L_{1}}(z)=H_{K}(z)+H_{L_{1}}(z) \leqslant H_{K}(z)<H_{D}(z), \quad z \notin \Pi, \quad z \neq 0 .
$$

Следовательно, $K+L_{1} \subset D$. Пусть теперь $\tau_{0}=\pi / 2$. Точка $\tilde{z}=\exp \left(i\left(\alpha_{0}+\tau_{0}\right)\right)$ принадлежит $F_{1} \subset J(D)$. Если $F_{1} \subset \operatorname{int} J(D)$, то найдется $\gamma \in(0, \pi / 8)$ (не зависящее от $K$ и $d$ ) такое, что

$$
H_{D}(\exp (i \alpha))=+\infty>2 d+d_{1}, \quad \alpha \in\left[\alpha_{0}+\pi / 2, \alpha_{0}+2 \gamma+\pi / 2\right),
$$

где $d_{1}=\max _{y \in \mathrm{S}} H_{K}(y)$. В противном случае в силу непрерывности функции $H_{D}$ (в широком смысле) в точке $\tilde{z}$ найдется $\gamma \in(0, \pi / 8)$ (уже зависяшее от $K$ и $d$ ) такое, что

$$
H_{D}(\exp (i \alpha))>2 d+d_{1}, \quad \alpha \in\left[\alpha_{0}+\pi / 2, \alpha_{0}+2 \gamma+\pi / 2\right) .
$$

Положим

$$
\begin{gathered}
\Pi_{1}=\left\{t \exp (i \alpha): \alpha \in\left(\alpha_{0}+2 \gamma-\pi / 2, \alpha_{0}+2 \gamma+\pi / 2\right), t>0\right\}, \\
E=\left\{\exp (i \alpha): \alpha \in\left(\alpha_{0}+3 \gamma-\pi / 2, \alpha_{0}+\gamma+\pi / 2\right)\right\} .
\end{gathered}
$$

Легко видеть, что множество $E$ является окрестностью $F_{1}$ на $\mathbf{S}$. Пусть $\zeta=$ $\exp \left(-i\left(\alpha_{0}+2 \gamma\right)\right)$ и $t_{2}>0$ такое, что

$$
t_{2} \operatorname{Re}\left(\zeta \exp \left(i\left(\alpha_{0}+\gamma+\pi / 2\right)\right)\right)=t_{2} \sin \gamma=d .
$$

Тогда для компакта $L_{1}=\left\{t_{2} \zeta\right\}$ имеем

$$
\begin{array}{ll}
H_{L_{1}}(z)=\operatorname{Re}\left(t_{2} \zeta z\right) \geqslant t_{2} \sin \gamma=d, & z \in E, \\
H_{L_{1}}(z)=\operatorname{Re}\left(t_{2} \zeta z\right) \leqslant t_{2} \sin 2 \gamma \leqslant 2 d, & z \in\left(\Pi_{1} \backslash \Pi\right) \cap \mathbf{S} .
\end{array}
$$

Кроме того, из предыдущего и неравенства $\operatorname{Re}(\zeta z) \leqslant 0, z \notin \Pi_{1}$, получаем

$$
\begin{array}{ll}
H_{K}(z)+H_{L_{1}}(z)<H_{D}(z)=+\infty, & z \in \Pi, \\
H_{K}(z)+H_{L_{1}}(z) \leqslant\left(2 d+d_{1}\right)|z|<H_{D}(z), & z \in\left(\Pi_{1} \backslash \Pi\right), \\
H_{K}(z)+H_{L_{1}}(z) \leqslant H_{K}(z)<H_{D}(z), & z \notin \Pi_{1}, \quad z \neq 0 .
\end{array}
$$

Отсюда следует, что $K+L_{1} \subset D$. Аналогичным образом строим компакт $L_{2}$ для множества $F_{2}$. Тогда, как и выше, компакт $L$, являюшийся выпуклой оболочкой объединения $L_{1} \cup L_{2}$, будет искомым. Лемма доказана.

Для $D \neq \mathbb{C}$ введем обозначения:

$$
\begin{aligned}
S_{\Lambda}(F) & =\sup _{E \supset F} S_{\Lambda(E)}, & S_{\Lambda}(D) & =\inf _{F \subset(\mathbf{S} \backslash J(D))} S_{\Lambda}(F), \\
M_{\Lambda}(F) & =\sup _{E \supset F} M_{\Lambda(E)}, & M_{\Lambda}(D) & =\inf _{F \subset(\mathbf{S} \backslash J(D))} M_{\Lambda}(F),
\end{aligned}
$$

где $E$ - произвольное открытое на $\mathbf{S}$ множество, содержащее $F$, а $F$ - компактное подмножество $\mathbf{S} \backslash J(D)$. Для $\Lambda=\left\{\lambda_{k}, m_{k}\right\}$ через $N(\Lambda)$ обозначим совокупность всех предельных точек последовательности $\left\{\lambda_{k} /\left|\lambda_{k}\right|\right\}, \lambda_{k} \neq 0$. 
ТЕОРЕМА 3.7. Пусть выпуклая область $D$ u $\Lambda=\left\{\lambda_{k}, m_{k}\right\}$ maкuе, что $I_{\Lambda}(D) \neq\{0\}$. Предположим, что выполнено следующее:

1) $S_{\Lambda} \geqslant a>-\infty$

2) $S_{\Lambda}(D)=0$;

3) существуют функиия $\varphi_{0} \in I_{\Lambda}(D)$ и $\alpha_{0} \in \mathbb{R}$ такие, что для любых $\delta>0$ $u w \in(N(\Lambda) \cap \partial J(D)) \cap J(D)$ найдутся $\beta, T>0$, удовлетворяющие условию: $\lambda_{k} \in R_{0}^{\delta}$, если $\lambda_{k} /\left|\lambda_{k}\right| \in B(w, \beta) u\left|\lambda_{k}\right|>T$, əде $R_{0}=\left\{z: \ln \left|\varphi_{0}(z)\right| \geqslant a_{0}|z|\right\} ;$

4) для каждого $m \geqslant 1$ существует $\varphi \in I_{\Lambda}(D)$ такая, что для любых $\delta>0$ $u w \in(N(\Lambda) \cap \partial J(D)) \backslash J(D)$ найдутся $\beta, T>0$, удовлетворяющие условию: $\lambda_{k} \in R_{m}^{\delta}$, если $\lambda_{k} /\left|\lambda_{k}\right| \in B(w, \beta) u\left|\lambda_{k}\right|>T$, zде $R_{m}=\left\{z: \ln |\varphi(z)| \geqslant H_{K_{m}}(z)\right\}$;

5) для любых $m \geqslant 1, \delta>0$ и каждого компактного подмножества $F$ множества $\mathbf{S} \backslash \overline{J(D)}$ существуют $\varphi \in I_{\Lambda}(D)$ u $T>0$ mакие, что $\lambda_{k} \in R_{m}^{\delta}$, если $\lambda_{k} /\left|\lambda_{k}\right| \in F u\left|\lambda_{k}\right|>T$.

Тогда:

(i) $M_{\Lambda}(D)=m_{D}(\Lambda)=0$;

(ii) $\Sigma: \mathbf{P}_{D} / I_{\Lambda}(D) \rightarrow \Phi-$ изоморфизм.

ЗАМЕЧАнИЕ 3.8. Условие 1) обеспечивает то, что в относительной близости от каждого $\lambda_{k}$ находится лишь определенное число других точек $\lambda_{p}$ и лишь на определенных расстояниях. Условие 2) аналогично условию 1), но является более сильным ограничением и относится только к тем $\lambda_{k}$, которые лежат на множестве, где $H_{D}$ конечна, а также сосредоточены вдоль одного или двух граничных лучей множества $J(D)$, не принадлежащих ему (если такие существуют). За исключением двух случаев, когда $D$ - полоса или полуплоскость, граничные лучи множества $J(D)$ являются и граничными лучами дополнительного множества, где $H_{D}$ конечна. В исключительных случаях дополнительное к $J(D)$ множество просто совпадает с граничными лучами $J(D)$.

ЗАМЕчАНИЕ 3.9. Условия 3)-5) накладывают ограничения на рост функций $\varphi \in I_{\Lambda}(D)$ в относительной близости от тех $\lambda_{k}$, которые лежат на множестве, где $H_{D}$ конечна, а также сосредоточены вдоль граничных лучей $J(D)$. Эти ограничения означают в каком-то смысле равномерное распределение указанных $\lambda_{k}$.

ЗАМЕЧАНИЕ 3.10. Условия 3) и 4) фактически отсутствуют, если пусты соответственно множества $(N(\Lambda) \cap \partial J(D)) \cap J(D)$ и $(N(\Lambda) \cap \partial J(D)) \backslash J(D)$. В случае, когда $D$ ограничена или совпадает со всей плоскостью, оба эти множества пусты. Если $D$ ограничена, то условие 1 ) является следствием условия 2 ). Если же $D=\mathbb{C}$, то из всех условий остается только первое.

ДоКАЗАТЕЛЬСТВО ТЕОРЕМЫ 3.7. (i) Пусть $F$ - компактное подмножество $\mathbf{S} \backslash J(D)$ и $E(\beta)$ - его $\beta$-окрестность. Если $\Lambda(E(\beta))$ для некоторого $\beta>0$ имеет конечное число элементов, то по определению $M_{\Lambda}(F)=0$. Будем полагать поэтому, что $\Lambda(E(\beta))$ бесконечна для всех $\beta>0$. Покажем, что

$$
M_{\Lambda(E(\beta))} \rightarrow 0, \quad \beta \rightarrow 0 .
$$

Предположим противное. Тогда с учетом (3.3) для некоторого $\tilde{\varepsilon}>0$ найдутся подпоследовательность $\left\{\tilde{\lambda}_{p}\right\}$ последовательности $\left\{\lambda_{k}\right\}$ и точка $x_{0} \in F$ такие, что $\left|\tilde{\lambda}_{p}\right| \rightarrow \infty, \tilde{\lambda}_{p} /\left|\tilde{\lambda}_{p}\right| \in B\left(x_{0}, 1 / p\right)$ и

$$
M_{\Lambda}\left(\tilde{\lambda}_{p}, 1 / p\right)>\tilde{\varepsilon}\left|\tilde{\lambda}_{p}\right|, \quad p=1,2, \ldots
$$


Пусть вначале $x_{0} \in \mathbf{S} \backslash \overline{J(D)}$. Тогда, как мы уже отмечали ранее, функция $H_{D}$ непрерывна (в обычном смысле) в некоторой окрестности точки $x_{0}$. Следовательно, для каждого $\varepsilon>0$ найдется $\tau \in(0,1 / 2)$ такое, что

$$
\left|H_{D}(\zeta)-H_{D}(w)\right| \leqslant \varepsilon, \quad w \in B\left(x_{0}, \tau\right), \quad \zeta \in B(w, 2 \tau) .
$$

Поскольку $H_{D}$ непрерывна на $\overline{B\left(x_{0}, \tau\right)}$, а компакты $K_{m}$ исчерпывают область $D$, то найдется номер $m$ такой, что

$$
H_{K_{m}}(x) \geqslant H_{D}(x)-\varepsilon, \quad x \in \overline{B\left(x_{0}, \tau\right)} .
$$

Пусть $\delta \in(0, \tau / 3), F=\overline{B\left(x_{0}, \tau\right)} \cap \mathbf{S}$ и $\varphi \in I_{\Lambda}(D)$ определяется по $m, \delta, F$ в условии 5). Из включения $\varphi \in I_{\Lambda}(D) \subset \mathbf{P}_{D}$ следует, что $h_{\varphi}(z)<H_{D}(z), z \neq 0$. Тогда с учетом (3.19) по лемме 3.2 для некоторого $t>0$ получаем

$$
M_{\Lambda}(y, 2 \delta) \leqslant H_{D}(y)+\varepsilon|y|-\ln |\varphi(y)| \quad \forall y: y /|y| \in B\left(x_{0}, \tau\right), \quad|y|>t, \quad \varphi(y) \neq 0 .
$$

Используя эту оценку, $(3.20)$ и определение $R_{m}$, имеем

$$
M_{\Lambda}(y, 2 \delta) \leqslant 2 \varepsilon|y|, \quad y /|y| \in B\left(x_{0}, \tau\right), \quad y \in R_{m}, \quad|y|>t .
$$

Так как $\tilde{\lambda}_{p} /\left|\tilde{\lambda}_{p}\right| \rightarrow x_{0}$ и $\left|\tilde{\lambda}_{p}\right| \rightarrow \infty$, то по условию 5) для каждого $p \geqslant p_{0}$ найдется $y_{p} \in R_{m}$ такое, что круг $B\left(y_{p}, \delta\left|y_{p}\right|\right)$ содержит $\tilde{\lambda}_{p}$. Легко видеть, что $y_{p} /\left|y_{p}\right| \in$ $B\left(x_{0}, 2 \delta+1 / p\right), p \geqslant p_{0}$. Увеличивая при необходимости $p_{0}$, можно считать, что $y_{p} /\left|y_{p}\right| \in B\left(x_{0}, \tau\right), B\left(\tilde{\lambda}_{p}, p^{-1}\left|\tilde{\lambda}_{p}\right|\right) \subset B\left(y_{p}, 2 \delta\left|y_{p}\right|\right)$ и $\left|y_{p}\right|>t$ при $p \geqslant p_{0}$. Тогда из (3.3) и (3.21) получаем

$$
M_{\Lambda}\left(\tilde{\lambda}_{p}, 1 / p\right) \leqslant M_{\Lambda}\left(y_{p}, 2 \delta\right) \leqslant 2 \varepsilon\left|y_{p}\right|, \quad p \geqslant p_{0} .
$$

В силу произвольности $\varepsilon>0$ это противоречит (3.18), так как $\left|\tilde{\lambda}_{p}\right| \geqslant(1-\delta)\left|y_{p}\right|$.

Пусть теперь $x_{0} \in \partial J(D) \backslash J(D)$. Поскольку $x_{0}$ - предельная точка последовательности $\left\{\tilde{\lambda}_{p} /\left|\tilde{\lambda}_{p}\right|\right\}$, то $x_{0} \in N(\Lambda)$. Для каждого $\varepsilon>0$ выберем $m$ такое, что $H_{K_{m}}\left(x_{0}\right) \geqslant H_{D}\left(x_{0}\right)-\varepsilon$. Пусть $\varphi \in I_{\Lambda}(D)$ удовлетворяет условию 4$)$. В силу непрерывности $h_{\varphi}$ с учетом неравенства $h_{\varphi}\left(x_{0}\right)<H_{D}\left(x_{0}\right)$ найдем $\tau \in(0,1 / 3)$ такое, что $h_{\varphi}(x)<H_{D}\left(x_{0}\right), x \in B\left(x_{0}, 3 \tau\right)$. Имея в виду еше непрерьвность $H_{K_{m}}$, можно считать, что $H_{K_{m}}(x) \geqslant H_{D}\left(x_{0}\right)-2 \varepsilon, x \in B\left(x_{0}, 3 \tau\right)$. По условию 4$)$ для каждого $\delta \in(0, \tau / 3)$ любая точка $\tilde{\lambda}_{p}, p \geqslant p(\delta)$, принадлежит $R_{m}^{\delta}$, т. е. для некоторого $y_{p} \in R_{m}$ попадает в круг $B\left(y_{p}, \delta\left|y_{p}\right|\right)$. Тогда при больших $p$ верно вложение $B\left(\tilde{\lambda}_{p}, p^{-1}\left|\tilde{\lambda}_{p}\right|\right) \subset B\left(y_{p}, 2 \delta\left|y_{p}\right|\right)$. Кроме того, поскольку $\left\{\tilde{\lambda}_{p} /\left|\tilde{\lambda}_{p}\right|\right\}$ сходится к $x_{0}$, то $y_{p} /\left|y_{p}\right| \in B\left(x_{0}, \tau\right)$ при больших $p$. По лемме 3.2 имеем

$$
M_{\Lambda}(y, 2 \delta) \leqslant H_{D}\left(x_{0}\right)|y|+\varepsilon|y|-\ln |\varphi(y)| \quad \forall y: y /|y| \in B\left(x_{0}, \tau\right), \quad|y|>t_{1} .
$$

Проводя далее такие же рассуждения, как и в первом случае, мы вновь получим противоречие с (3.18).

Таким образом, мы показали, что (3.17) выполнено. Тогда по определению $M_{\Lambda}(F)=0$, а в силу произвольности $F$ и $M_{\Lambda}(D)=0$. С учетом очевидных неравенств $M_{\Lambda}(D) \geqslant m_{D}(\Lambda) \geqslant 0$ получаем первое утверждение теоремы. 
(ii) Согласно доказанному и предложениям 2.4, 2.3 оператор $\Sigma: \mathbf{P}_{D} / I_{\Lambda}(D) \rightarrow \Phi$ непрерывен и инъективен. В силу теоремы об открытом отображении достаточно показать теперь, что он является сюръективным. Пусть $b=\left\{b_{k, n}\right\}-$ произвольный элемент пространства $\Phi$, а значит, $\Phi_{s}$ для некоторого $s \geqslant 1$. Нам нужно лишь построить функцию $f \in \mathbf{P}_{D}$ такую, что $f^{(n-1)}\left(\lambda_{k}\right)=b_{k, n}, n=1, \ldots, m_{k}$, $k \geqslant 1$. Другими словами, необходимо аналитически продолжить функцию, заданную вместе со своими производными в точках последовательности $\left\{\lambda_{k}\right\}$, до целой функции с подходящими оценками на рост. Это продолжение мы будем осуществлять в три этапа: локальное продолжение, полулокальное продолжение и собственно продолжение до целой функции.

1. Для каждого $k=1,2, \ldots$ положим

$$
\psi_{k}(\lambda)=\sum_{n=1}^{m_{k}} \frac{b_{k, n}}{(n-1) !}\left(\lambda-\lambda_{k}\right)^{n-1} .
$$

По построению производная порядка $n$ функции $\psi_{k}$ в точке $\lambda_{k}$ равна $b_{k, n}, \quad n=$ $1, \ldots, m_{k}$. Таким образом, функцию, заданную первоначально вместе со своими производными лишш в точках $\lambda_{k}$, мы продолжили в окрестность каждой из этих точек. Теперь необходимо установить, как такое продолжение изменило исходные оценки. Имеем

$$
\begin{aligned}
& \frac{1}{\left|\lambda_{k}\right|} \ln \left|\frac{\left(\lambda-\lambda_{k}\right)^{n-1}}{(n-1) !}\right| \leqslant \frac{1}{\left|\lambda_{k}\right|} \ln \left|\frac{3\left(\lambda-\lambda_{k}\right)}{n-1}\right|^{n-1} \leqslant \frac{n-1}{\left|\lambda_{k}\right|} \ln \left|\frac{3 \lambda_{k}}{n-1}\right| \\
& \leqslant \sup _{x>0} x^{-1} \ln (3 x) \leqslant 2, \quad \lambda \in B\left(\lambda_{k},\left|\lambda_{k}\right|\right), \quad k=1,2, \ldots, \quad n=2, \ldots, m_{k}, \quad \lambda_{k} \neq 0 .
\end{aligned}
$$

Следовательно, с учетом определения пространства $\Phi_{s}$ и неравенства $m_{k} \leqslant C\left|\lambda_{k}\right|$ (которым мы уже пользовались выше) получаем

$$
\begin{gathered}
\left|\psi_{k}(\lambda)\right| \leqslant C\|b\|_{s} m_{k} \exp \left(H_{K_{s}}\left(\lambda_{k}\right)+2\left|\lambda_{k}\right|\right) \leqslant C_{1} \exp \left((\gamma+3)\left|\lambda_{k}\right|\right), \\
\lambda \in B\left(\lambda_{k},\left|\lambda_{k}\right|\right), \quad k=1,2, \ldots,
\end{gathered}
$$

где $\gamma=\max _{z \in \mathrm{S}} H_{K_{s}}(z)$. Эти оценки достаточно грубы, но верны для всех $k$. Для некоторых $k$ можно получить более точные оценки. Пусть $F$ - компактное подмножество $\mathbf{S} \backslash J(D), \quad E(\beta)$ - его окрестность и $\varepsilon>0$. Покажем, что для некоторых $C_{2}, \beta_{0}>0$ верно неравенство

$$
\left|\psi_{k}(\lambda)\right| \leqslant C_{2} \exp \left(H_{K_{s}}\left(\lambda_{k}\right)+\varepsilon\left|\lambda_{k}\right|\right), \quad \lambda \in B\left(\lambda_{k},\left|\lambda_{k}\right|\right), \quad \lambda_{k} /\left|\lambda_{k}\right| \in E\left(\beta_{0}\right)
$$

Пусть $\tilde{\varepsilon}>0$. В силу (3.17) существуют $\beta_{0}$ и $k_{0}$ такие, что при $k \geqslant k_{0}$ и $\lambda_{k} /\left|\lambda_{k}\right| \in$ $E\left(\beta_{0}\right)$ имеет место оценка $m_{k} /\left|\lambda_{k}\right| \leqslant \tilde{\varepsilon}$. Отсюда, как и в $(2.10)$, при подходяшем $\tilde{\varepsilon}>0$ и тех же $k$ и $\lambda_{k}$ получаем

$$
\left|\frac{\left(\lambda-\lambda_{k}\right)^{n-1}}{(n-1) !}\right| \leqslant \exp \left(\frac{\varepsilon\left|\lambda_{k}\right|}{2}\right), \quad n=1, \ldots, m_{k} .
$$

Это неравенство вместе с оценкой $m_{k} \leqslant \tilde{\varepsilon}\left|\lambda_{k}\right|$ обеспечивает (3.23). 
2. На этом этапе построим функции, которые вместе со своими производными принимают заданные значения уже не в одной лишь точке $\lambda_{k}$, а на группе таких точек. Фиксируем $\varepsilon>0$. Как было отмечено выше, величина $M_{\Lambda}$ конечна. Пусть $M_{\Lambda} \leqslant A$. По лемме 3.1 найдем $\delta_{0} \in(0,1 / 3)$ такое, что для всех $\delta \in\left(0, \delta_{0}\right)$ выполнено неравенство из утверждения этой леммы. Фиксируем $\tilde{\delta} \in\left(0, \delta_{0} / 8\right)$, и пусть $k \geqslant 1$. Легко видеть, что для каждого $\lambda_{j} \in B\left(\lambda_{k}, 2 \tilde{\delta}\left|\lambda_{k}\right|\right)$ круг $B\left(\lambda_{j}, 8 \tilde{\delta}\left|\lambda_{j}\right|\right)$ содержит $B\left(\lambda_{k}, 2 \tilde{\delta}\left|\lambda_{k}\right|\right)$. Поэтому согласно (3.2)

$$
\left|q_{\Lambda}\left(z, \lambda_{k}, 2 \tilde{\delta}\right)\right| \geqslant\left|q_{\Lambda}\left(z, \lambda_{j}, 8 \tilde{\delta}\right)\right|, \quad z \in B\left(\lambda_{j}, 8 \tilde{\delta}\left|\lambda_{j}\right|\right), \quad \lambda_{j} \in B\left(\lambda_{k}, 2 \tilde{\delta}\left|\lambda_{k}\right|\right)
$$

В силу выбора величин $\rho_{k}(\tilde{\delta})$ в лемме 3.1 круги $B\left(\lambda_{j}, 2^{-1} \rho_{j}(8 \tilde{\delta})\right), j=1,2, \ldots$, попарно не пересекаются. Для каждого $j=1,2, \ldots$ через $e_{j}$ обозначим функцию, обладаюшую следуюшими свойствами (здесь $\widetilde{C}_{2}$ и $d_{j}-$ те же, что и в лемме 3.1 ):

1) $e_{j} \in C^{\infty}(\mathbb{C})$

2) $0 \leqslant e_{j}(z) \leqslant 1 \forall z \in \mathbb{C}$

3) $e_{j}(z)=1, z \in B\left(\lambda_{j}, 2^{-1} \rho_{j}(8 \tilde{\delta})-\widetilde{C}_{2} d_{j}\right)$;

4) $e_{j}(z)=0, z \notin B\left(\lambda_{j}, 2^{-1} \rho_{j}(8 \tilde{\delta})\right)$;

5) $\left|d e_{j}(z) / d \bar{z}\right| \leqslant \widetilde{C}_{3} d_{j}^{-1} \forall z \in \mathbb{C}$, где $\widetilde{C}_{3}>0$ не зависит от $j=1,2, \ldots$ (о построении таких функций см., например, [51, теорема 1.4 .1 и формула (1.4.2)]).

Фиксируем $k=1,2, \ldots$ Из свойств 3$)$ и 4 ) следует, что функция

$$
\sigma_{k}(z)=q_{\Lambda}^{-1}\left(z, \lambda_{k}, 2 \delta\right) \sum_{j} \psi_{j}(z) \frac{d e_{j}(z)}{d \bar{z}}
$$

где суммирование ведется по всем $j$ таким, что $\lambda_{j} \in B\left(\lambda_{k}, \tilde{\delta}\left|\lambda_{k}\right|\right)$, отлична от нуля лишь в кольцах $2^{-1} \rho_{j}(8 \tilde{\delta})-\widetilde{C}_{2} d_{j} \leqslant\left|z-\lambda_{j}\right| \leqslant 2^{-1} \rho_{j}(8 \tilde{\delta})$ для тех же $j$. Из свойства 5$)$ для $e_{j}$, неравенств $(3.22),(3.24)$ и леммы 3.1 получаем

$$
\begin{gathered}
\left|\sigma_{k}(z)\right| \leqslant C_{1} \widetilde{C}_{1}^{-1} \widetilde{C}_{3} d_{p}^{-2} \exp \left((\gamma+3)\left|\lambda_{k}\right|\right), \\
2^{-1} \rho_{p}(8 \tilde{\delta})-\widetilde{C}_{2} d_{p} \leqslant\left|z-\lambda_{p}\right| \leqslant 2^{-1} \rho_{p}(8 \tilde{\delta}) .
\end{gathered}
$$

Уменьшая при необходимости $\tilde{\delta}$ и используя (3.13), имеем

$$
\left|\sigma_{k}(z)\right| \leqslant \widetilde{C}_{4} d_{p}^{-2} \exp ((3 A-2 a+\gamma+3+4 \varepsilon)|z|) \quad \forall z,
$$

где $\widetilde{C}_{4}>0$ не зависит от $k=1,2, \ldots$ Отсюда следует, что

$$
\int_{\mathbb{C}}\left|\sigma_{k}(z)\right|^{2} \exp ((4 a-6 A-2 \gamma-6-10 \varepsilon)|z|) d \lambda(z) \leqslant \widetilde{C}_{5}, \quad k=1,2, \ldots
$$

Тогда, как известно (см., например, [ 52, гл. $3, \S 6$, п. 2 , теорема 3.6 .2$])$, в пространстве локально интегрируемых с квадратом модуля функций в $\mathbb{C}$ найдется элемент $g$, который (в обобшенном смысле) удовлетворяет равенству

$$
\frac{d g_{k}}{d \bar{z}}=\sigma_{k}, \quad k=1,2, \ldots,
$$


и, кроме того, оценке

$$
\begin{aligned}
\int_{\mathbb{C}}\left|\sigma_{k}(z)\right|^{2} \exp ((4 a-6 A-2 \gamma-6-12 \varepsilon)|z|) d \lambda(z) \\
\quad \leqslant \int_{\mathbb{C}}\left|\sigma_{k}(z)\right|^{2} \exp ((4 a-6 A-2 \gamma-6-10 \varepsilon)|z|)\left(1+|z|^{2}\right)^{-2} d \lambda(z) \leqslant 2^{-1} \widetilde{C}_{5} .
\end{aligned}
$$

Для каждого $k=1,2, \ldots$ рассмотрим функцию

$$
\tilde{\psi}_{k}(z)=\sum_{j} \psi_{j}(z) e_{j}(z)-g_{k}(z) q_{\Lambda}\left(z, \lambda_{k}, 2 \delta\right)
$$

где суммирование по $j$ такое же, как и вьше. Легко видеть, что $\tilde{\psi}_{k}$ вместе со своими производными принимает значения $b_{j, n}$ в точках $\lambda_{j} \in B\left(\lambda_{k}, \tilde{\delta}\left|\lambda_{k}\right|\right)$. В силу (3.25) обобщенная производная $\tilde{\psi}_{k}$ по $\bar{z}$ равна нулю всюду в плоскости. Хорошо известно, что это означает аналитичность $\tilde{\psi}_{k}(z)$. Найдем теперь оценку на $\left|\tilde{\psi}_{k}\right|$ в круге $B\left(\lambda_{k}, \tilde{\delta}\left|\lambda_{k}\right|\right)$. Выберем $k_{0}$ такое, что $\tilde{\delta}\left|\lambda_{k}\right| \geqslant 1$ при $k \geqslant k_{0}$. Напомним, что модуль функции $q_{\Lambda}\left(z, \lambda_{k}, 2 \tilde{\delta}\right)$ оценивается сверху единицей в круге $B\left(\lambda_{k}, 2 \tilde{\delta}\left|\lambda_{k}\right|\right)$, и в каждой точке $z$ отлична от нуля не более чем одна из функций $e_{j}$. С учетом этого, используя субгармоничность $\tilde{\psi}_{k}$, свойство 2) для $e_{j}$ и неравенства $(3.22),(3.13)$, получаем для всех $z \in B\left(\lambda_{k}, \tilde{\delta}\left|\lambda_{k}\right|\right)$ и $k \geqslant k_{0}$

$$
\begin{aligned}
\left|\tilde{\psi}_{k}(z)\right| & \leqslant \pi^{-1} \int_{B(z, 1)}\left|\tilde{\psi}_{k}(y)\right| d \lambda(y) \\
& \leqslant \pi^{-1} \int_{B(z, 1)}\left|g_{k}(y)\right| d \lambda(y)+C_{1} \exp ((\gamma+3+\varepsilon)|z|) .
\end{aligned}
$$

Отсюда, используя еше (3.26) и неравенство Коши-Буняковского, имеем

$$
\begin{aligned}
\left|\tilde{\psi}_{k}(z)\right| \leqslant & C_{1} \exp ((\gamma+3+\varepsilon)|z|) \\
& +\pi^{-1}\left(\frac{1}{2} \widetilde{C}_{5} \int_{B(z, 1)} \exp ((6 A-4 a+2 \gamma+6+12 \varepsilon)|y|) d \lambda(y)\right)^{1 / 2} \\
\leqslant & C_{1} \exp ((\gamma+3+\varepsilon)|z|) \\
& +\left(2^{-1} \widetilde{C}_{5}\right)^{1 / 2} \exp \left(\sup _{B(z, 1)}((3 A-2 a+\gamma+3+6 \varepsilon)|y|)\right) \\
\leqslant & C_{3} \exp ((3 A-2 a+\gamma+3+7 \varepsilon)|z|), \quad z \in B\left(\lambda_{k}, \tilde{\delta}\left|\lambda_{k}\right|\right) .
\end{aligned}
$$

Увеличивая при необходимости $C_{3}$, можно считать, что последнее неравенство выполнено для всех $k=1,2, \ldots$

Пусть $F$ - компактное подмножество $\mathbf{S} \backslash J(D)$ и $E(\beta)$ - его окрестность. Как и выше, для номеров $k$ таких, что $\lambda_{k} /\left|\lambda_{k}\right| \in E(\beta)$, можно получить более точные оценки. Для этого в приведенных выше рассуждениях достаточно использовать (3.23) вместо (3.22) и то, что для некоторого $\beta>0$ в силу условия 2$)$ теоремы $S_{\Lambda(E(\beta))} \geqslant-\varepsilon$, а в силу уже доказанного утверждения (i) $M_{\Lambda(E(\beta))} \leqslant \varepsilon$. Таким образом, подбирая подходящие $\tilde{\beta}, \tilde{\delta}>0$, имеем

$$
\left|\tilde{\psi}_{k}(z)\right| \leqslant C_{4} \exp \left(H_{K_{s}}(z)+13 \varepsilon|z|\right), \quad z \in B\left(\lambda_{k}, \tilde{\delta}\left|\lambda_{k}\right|\right), \quad \lambda_{k} /\left|\lambda_{k}\right| \in E(\tilde{\beta}) .
$$


3. На заключительном этапе доказательства построим требуемую целую функцию. Разобьем этот этап на несколько пунктов. Их число зависит от вида области $D$. Поясним это подробнее. В обшем случае существует четыре типа точек: точки, в окрестности которых функция $H_{D}$ конечна, точки, в окрестности которых она бесконечна, и точки, в окрестности которых она и конечна и бесконечна одновременно, но последние, в свою очередь, бывают двух видов: точки, где $H_{D}$ принимает значение $+\infty$, и точки, где $H_{D}$ конечна. В частных случаях присутствуют не все точки указанных типов. $\mathrm{K}$ примеру, если $D$ ограничена или совпадает со всей плоскостью, то сушествуют точки только одного типа (своего для каждого из случаев). Если $D$ - полуплоскость или полоса, то существует только два типа точек. Если же $D$ - область, ограниченная параболой, то имеется три типа точек. Мы рассмотрим, естественно, самьй общий случай, когда существуют точки всех типов, т. е. когда $D$ - неограниченная область, и одна из точек $\partial J(D) \cap \mathbf{S}$, скажем $w_{2}$, принадлежит множеству $J(D)$, а другая, скажем $w_{1}$, не принадлежит ему. Такая ситуация реализуется, например, для области, лежащей в верхней полуплоскости, граница которой в левой полуплоскости совпадает с куском параболы, а в правой полуплоскости - с положительной вешественной полуосью. Кроме того, мы считаем, что обе точки, $w_{1}$ и $w_{2}$, содержатся в $N(\Lambda)$ (в противном случае приводимые ниже рассуждения упрошаются).

Этот этап доказательства разобьем на четыре пункта, в каждом из которьх будем строить целую функцию, принимающую вместе со своими производными заданные значения лишь в точках $\lambda_{k}$ таких, что $\lambda_{k} /\left|\lambda_{k}\right|$ лежит в окрестности множества, состояшего из точек одного типа. Требуемую функцию мы получим затем, сложив эти целые функции. В частных случаях некоторые пункты могут отсутствовать, если отсутствуют точки соответствующего типа.

3.1. Фиксируем $\varepsilon \in\left(0, \alpha_{s} / 100\right)$, где $\alpha_{s}$ - такое же, как и в (1.1). Выберем номер $m \geqslant s+1$ такой, что $H_{K_{m}}\left(w_{1}\right) \geqslant H_{D}\left(w_{1}\right)-\varepsilon$. Пусть $\varphi \in I_{\Lambda}(D)$ удовлетворяет условию 4$)$. В силу непрерывности $H_{K_{m}}$ и $h_{\varphi}$, как и выше, для некоторого $\alpha \in$ $(0,1 / 6)$ имеем

$$
h_{\varphi}(z) \leqslant H_{D}\left(w_{1}\right) \leqslant H_{K_{m}}(z)+2 \varepsilon, \quad z \in B\left(w_{1}, 6 \alpha\right) .
$$

Пусть $h(z)=H_{K_{m}}(z)$ и $H(z)=H_{K_{m}}(z)+2 \varepsilon|z|$. По функциям $h(z), H(z)$ и числам $\varepsilon, \alpha$ найдем $\delta_{0}>0$ из леммы 3.3 и фиксируем положительное $\delta<$ $\min \left\{\delta_{0}, \alpha / 22, \tilde{\delta} / 88\right\} \quad(\tilde{\delta}>0$ - такое же, как и в $(3.28)$, где в качестве $F$ берем одноточечное множество $\left.\left\{w_{1}\right\}\right)$. Согласно условию 4$)$ существуют $\beta \in(0, \alpha)$ и $T^{\prime}>0$ такие, что $\lambda_{k} \in B\left(z_{k}, \delta\left|z_{k}\right|\right)$ для некоторого $z_{k} \in R_{m}$, как только $\left|\lambda_{k}\right|>T^{\prime}$ и $\lambda_{k} /\left|\lambda_{k}\right| \in B\left(w_{1}, \beta\right)$. Легко видеть, что $z_{k} /\left|z_{k}\right| \in B\left(w_{1}, 2 \alpha\right)$. Пусть множество $R$ состоит из всех точек $z_{k}$. Тогда в силу (3.29)

$$
h_{\varphi}(z) \leqslant H_{K_{m}}(z)+2 \varepsilon|z|, \quad z \in R^{\alpha} .
$$

Отсюда согласно определению $R_{m}$ по лемме 3.3 найдем $T>0$ и попарно непересекающиеся открытые множества $U_{p}, p \geqslant 1$, обладающие свойствами 1)-3) этой леммы и такие, что

$$
\begin{aligned}
\ln |\varphi(z)| & \geqslant h(z)-2 \varepsilon|z|-(3+\ln 24)(H(z)-h(z)+3 \varepsilon|z|) \\
& \geqslant h(z)-2 \varepsilon|z|-7(2 \varepsilon|z|+3 \varepsilon|z|)=H_{K_{m}}(z)-37 \varepsilon|z|, \quad z \in \partial U_{p} .
\end{aligned}
$$


Пусть $\widetilde{R}=\cup \partial U_{p}, h_{1}(z)=H_{K_{m}}(z)-37 \varepsilon|z|$ и $H_{1}(z)=H_{K_{m}}(z)+2 \varepsilon|z|$. В силу свойства 2$)$ из леммы 3.3 и выбора $\delta$ верно вложение $\widetilde{R} \subset R^{\alpha}$. Тогда, как нетрудно заметить, $z /|z| \in B\left(w_{1}, 6 \alpha\right)$ для всех $z \in \widetilde{R}^{\alpha}$. Следовательно, согласно (3.29) и (3.31) выполнены все условия леммы 3.5 , по которой имеем

$$
|\varphi(y)| \geqslant \exp \left(H_{K_{m}}(y)-40 \varepsilon|y|\right), \quad y \in B(z, \exp (-43 \varepsilon|z|)), \quad z \in \widetilde{R} \backslash B(0, \widetilde{T}) .
$$

Пусть $T_{1}>\max \left\{T^{\prime}, T, \widetilde{T} /(1-88 \delta)\right\}$ (не ограничивая общности, можно считать, что $88 \delta<1)$ и $\Gamma_{1}=\left\{t z: z \in B\left(w_{1}, \beta_{1}\right) \cap \mathbf{S}, t>T_{1}\right\}$, где $0<\beta_{1}<\min \{\beta, \tilde{\beta}\}$ $\left(\tilde{\beta}\right.$ такое же, как в (3.28)) и $w_{2} \notin \overline{B\left(w_{1}, \beta_{1}\right)}$. Согласно выбору $\beta_{1}$ и $T_{1}$ каждая точка $\lambda_{k}$ из $\Gamma_{1}$ принадлежит $R^{\delta}$, а значит, в силу свойства 3$)$ леммы 3.3 и некоторому $\bar{U}_{p}$. Пусть подпоследовательность $\left\{U_{p_{i}}\right\}$ состоит из всех множеств $U_{p}$ таких, что $\bar{U}_{p}$ содержит хотя бы одну точку $\lambda_{k} \in \Gamma_{1}$. Какую-нибудь одну из таких точек, принадлежаших $U_{p_{i}}$, обозначим $\lambda_{k_{i}}, i=1,2, \ldots$ По свойству 1) леммы 3.3 имеет место вложение $U_{p_{i}} \subset B\left(\lambda_{k_{i}}, 88 \delta\left|\lambda_{k_{i}}\right|\right), i \geqslant 1$. Следовательно, в силу выбора $\delta$ и $\beta_{1}$ для функции $\tilde{\psi}_{k_{i}}$ на множестве $U_{p_{i}}$ выполнено (3.28). Кроме того, из последнего вложения и выбора $T_{1}$ следует, что все $U_{p_{i}}$ лежат вне круга $B(0, \widetilde{T})$. Поэтому для каждого $i \geqslant 1$ и любого $z \in \partial U_{p_{i}}$ выполнено (3.32). Пусть

$$
V_{i}=U_{p_{i}} \backslash \cup\left\{B(z, \exp (-43 \varepsilon|z|)), z \in \partial U_{p_{i}}\right\}, \quad i=1,2, \ldots
$$

Поскольку $\varphi\left(\lambda_{k}\right)=0$, то в силу (3.32) каждая точка $\lambda_{k} \in \Gamma_{1}$ принадлежит внутренности одного из $V_{i}$ (так как принадлежит одному из $\bar{U}_{p_{i}}$ ). Уменьшая при необходимости $\delta>0$ (так, что при $\tau=88 \delta$ для функций $43 \varepsilon|z|$ и $44 \varepsilon|z|$ выполнено (3.13)), имеем

$$
\operatorname{dist}\left(V_{1}, \partial U_{p_{i}}\right) \geqslant \exp \left(-44 \varepsilon\left|\lambda_{k_{i}}\right|\right), \quad i \geqslant 1 .
$$

Тогда, как и вьше, найдем функции $e_{i}^{1}, i \geqslant 1$, обладаюшие следуюшими свойствами:

1) $e_{i}^{1} \in C^{\infty}(\mathbb{C})$

2) $0 \leqslant e_{i}^{1}(z) \leqslant 1 \forall z$

3) $e_{i}^{1}(z)=1, z \in V_{i}$

4) $e_{i}^{1}(z)=0, z \notin U_{\underline{C}_{i}}$

5) $\left|d e_{i}^{1}(z) / d \bar{z}\right| \leqslant \widetilde{C}_{6} \exp \left(44 \varepsilon\left|\lambda_{k_{i}}\right|\right) \quad \forall z$, где постоянная $\widetilde{C}_{6}>0$ не зависит от номера $i$.

С учетом (3.13) последнее свойство можно заменить на свойство

$\left.5^{\prime}\right)\left|d e_{i}^{1}(z) / d \bar{z}\right| \leqslant \widetilde{C}_{6} \exp (45 \varepsilon|z|) \forall z$.

Определим последовательность $b^{1}=\left\{b_{k, n}^{1}\right\}$. Положим $b_{k, n}^{1}=b_{k, n}, \quad n=$ $1, \ldots, m_{k}$, если $\lambda_{k} \in \Gamma_{1}$, и $b_{k, n}^{1}=0, n=1, \ldots, m_{k}$, если $\lambda_{k} \notin \Gamma_{1}$. Поскольку $\left\|b^{1}\right\|_{s} \leqslant\|b\|_{s}$, то будем считать, что все построения, которые мы провели выше, сделаны для последовательности $b^{1}$.

В силу свойств 3$)$ и 4) для $e_{i}^{1}$ функция $\nu_{1}(z)=\varphi^{-1}(z) \sum_{i} \tilde{\psi}_{k_{i}}(z) d e_{i}^{1}(z) / d \bar{z}$ отлична от нуля лишь на множествах $U_{p_{i}} \backslash V_{i}, i=1,2, \ldots$ Поэтому из свойства $5^{\prime}$ ) для $e_{i}^{1},(3.28)$ и $(3.32)$ получаем

$$
\left|\nu_{1}(z)\right| \leqslant C_{4} \widetilde{C}_{6} \exp \left(H_{K_{s}}(z)-H_{K_{m}}(z)+98 \varepsilon|z|\right) \quad \forall z .
$$


Отсюда с учетом (1.1), выбора $\varepsilon$ и $m$, а также вложения $K_{j} \subset K_{j+1}$ имеем

$$
\left|\nu_{1}(z)\right| \leqslant C_{4} \widetilde{C}_{6} \exp \left(H_{K_{s}}(z)-H_{K_{s+1}}(z)+98 \varepsilon|z|\right) \leqslant C_{4} \widetilde{C}_{6}
$$

Поскольку $\varphi \in \mathbf{P}_{D}$, то найдется $\varepsilon_{1}>0$ такое, что

$$
h_{\varphi_{1}}(z)+5 \varepsilon_{1}|z|<H_{D}(z), \quad z \neq 0 .
$$

По предыдущему имеем

$$
\int_{\mathbb{C}}\left|\nu_{1}(z)\right|^{2} \exp \left(-2 \varepsilon_{1}|z|\right) d \lambda(z)<\infty .
$$

Тогда, как и выше, найдем функцию $\theta_{1}(z)$, которая удовлетворяет уравнению $d \theta_{1}(z) / d \bar{z}=\nu_{1}(z)$ и неравенству $\int_{\mathbb{C}}\left|\theta_{1}(z)\right|^{2} \exp \left(-4 \varepsilon_{1}|z|\right) d \lambda(z)<\infty$. Положим

$$
f_{1}(z)=\sum_{i} \tilde{\psi}_{k_{i}}(z) e_{i}^{1}(z)-\theta_{1}(z) \varphi(z)
$$

Функция $f_{1}$ целая. Так же, как и для $\tilde{\psi}_{k}$, с учетом $(3.28)$, свойств 2$\left.), 4\right)$ функций $e_{i}^{1}$, неравенств (3.11) (где $\left.\tilde{\varepsilon}=\varepsilon_{1}\right)$ и (3.13) получаем оценку

$$
\begin{aligned}
\left|f_{1}(z)\right| & \leqslant \pi^{-1} \int_{B(z, 1)}\left|f_{1}(y)\right| d \lambda(y) \\
& \leqslant C_{4} \exp \left(H_{K_{s}}(z)+14 \varepsilon|z|\right)+\widetilde{C}_{7} \exp \left(h_{\varphi}(z)+2 \varepsilon_{1}|z|\right) \int_{\mathbb{C}}\left|\theta_{1}(y)\right| d \lambda(y) \\
& \leqslant C_{4} \exp \left(H_{K_{s+1}}(z)\right)+\widetilde{C}_{8} \exp \left(h_{\varphi}(z)+5 \varepsilon_{1}|z|\right) .
\end{aligned}
$$

Отсюда и из (3.33) следует, что

$$
h_{f_{1}}(z) \leqslant \max \left\{H_{K_{s+1}}(z), h_{\varphi}(z)+5 \varepsilon_{1}|z|\right\}<H_{D}(z), \quad z \neq 0
$$

т. е. $f_{1} \in \mathbf{P}_{D}$. По построению $f_{1}$ вместе со своими производными принимает значения $b_{k, n}^{1}$ в точках $\lambda_{k}$.

3.2. Построим функцию $f_{2} \in \mathbf{P}_{D}$, которая вместе со своими производными принимает значения $b_{k, n}^{2}$ в точках $\lambda_{k}$, где $b_{k, n}^{2}=b_{k, n}, n=\overline{1, m_{k}}$, если $\lambda_{k} \in \Gamma_{2}=$ $\left\{t z: z \in B\left(w_{2}, \beta_{2}\right) \cap \mathbf{S}, t>T_{2}\right\}$, и $b_{k, n}^{2}=0, n=\overline{1, m_{k}}$, если $\lambda_{k} \notin \Gamma_{2}$. Заранее полагаем, что $B\left(w_{1}, \beta_{1}\right) \cap B\left(w_{2}, \beta_{2}\right)=\varnothing$. Тогда $\Gamma_{1}$ и $\Gamma_{2}$ не пересекаются.

Пусть $\varepsilon, \alpha=1, h(z)=a_{0}|z|$ и $H(z)=d_{0}|z|$, где $d_{0}=\max _{y \in \mathrm{S}} h_{\varphi_{0}}(y)$. По $\varepsilon, \alpha, h$ и $H$ определим число $\delta_{0}$ из леммы 3.3. Положим $d=3 A-2 a+\gamma+65+15 d_{0}-16 a_{0}$ (где $A, a, \gamma$ - те же, что и в (3.27), и $a_{0}$ - из условия 3$)$ ), и пусть $K$ - сопряженная диаграмма $\varphi_{0}$, т. е. $H_{K} \equiv h_{\varphi_{0}}$. По лемме 3.6 для компакта $K$, множества $F=\left\{w_{2}\right\}$ и числа $d$ найдем $\beta>0$ и выпуклый компакт $L$ такие, что $K+L \subset D$ и $H_{L}(z) \geqslant d|z|$ $\forall z: z /|z| \in E(3 \beta)$. Уменьшая, если это необходимо, $\beta>0$, в силу непрерывности (в широком смысле) $H_{D}$ в точке $w_{2} \in J(D)$ можно считать, что

$$
H_{D}(z)>3 A-2 a+\gamma+10, \quad z \in \overline{B\left(w_{2}, 3 \beta\right)} .
$$


Тогда из определения опорной функции легко следует, что для некоторого компакта $L_{1} \subset D$ вьполнено неравенство

$$
H_{L_{1}}(z)>3 A-2 a+\gamma+10, \quad z \in \overline{B\left(w_{2}, 3 \beta\right)}
$$

Фиксируем положительное $\delta<\min \left\{\delta_{0}, \beta / 88, \tilde{\delta} / 88\right\} \quad(\tilde{\delta}>0$ такое же, как в (3.27)). Для $R=R_{0}$ по лемме 3.3 найдем $T>0$ и попарно непересекаюшиеся открытые множества $U_{p}, p \geqslant 1$, со свойствами 1)-3) леммы 3.3 такие, что

$$
\begin{aligned}
\ln \left|\varphi_{0}(z)\right| & \geqslant h(z)-2|z|-(3+\ln 24)(H(z)-h(z)+3|z|) \\
& \geqslant\left(8 a_{0}-7 d_{0}-23\right)|z|=d_{1}|z|, \quad z \in \partial U_{p}
\end{aligned}
$$

Пусть $\widetilde{R}=\cup \partial U_{p}, h_{1}(z)=d_{1}|z|$ и $H_{1}(z)=d_{0}|z|$. Тогда по лемме 3.5

$$
\left|\varphi_{0}(y)\right| \geqslant \exp \left(\left(d_{1}-3\right)|y|\right), \quad y \in B\left(z, \exp \left(\left(d_{1}-d_{0}-4\right)|z|\right)\right), \quad z \in \widetilde{R} \backslash B(0, \widetilde{T}) .
$$

Согласно условию 3$)$ сушествуют $\beta_{2} \in(0, \beta)$ и $T^{\prime}>0$ такие, что $\lambda_{k} \in R_{0}^{\delta}=R^{\delta}$, если $\left|\lambda_{k}\right|>T^{\prime}$ и $\lambda_{k} /\left|\lambda_{k}\right| \in B\left(w_{2}, \beta_{2}\right)$. Пусть $T_{2}>\max \left\{T^{\prime}, T, \widetilde{T} /(1-88 \delta)\right\}$ и $U_{p_{i}}$, $\lambda_{k_{i}}, i \geqslant 1$, обладают теми же свойствами, что и в п. 3.1 , но уже для множества $\Gamma_{2}$. Положим

$$
V_{i}=U_{p_{i}} \backslash \cup\left\{B\left(z, \exp \left(\left(d_{1}-d_{0}-4\right)|z|\right)\right), z \in \partial U_{p_{i}}\right\}, \quad i=1,2, \ldots
$$

Тогда, как и в п. 3.1, найдем функции $e_{i}^{2}, i \geqslant 1$, обладающие свойствами 1$\left.)-4\right)$ для $e_{i}^{1}$ и свойством

$\left.5^{\prime \prime}\right)\left|d e_{i}^{2}(z) / d \bar{z}\right| \leqslant \widetilde{C}_{9} \exp \left(\left(d_{0}+6-d_{1}\right)|z|\right) \forall z$, где $\widetilde{C}_{9}>0$ не зависит от $i$.

Функция $\sum \tilde{\psi}_{k_{j}} e_{j}^{2}$ отлична от нуля на множествах $U_{p_{i}} \subset B\left(\lambda_{k_{i}}, 88 \delta\left|\lambda_{k_{i}}\right|\right), i \geqslant 1$, и удовлетворяет в них такой же оценке, как и последняя оценка в (3.27). В силу выбора $\beta_{2}$ и $\delta$ для любых $i \geqslant 1$ и $z \in U_{p_{i}} \subset B\left(\lambda_{k_{i}}, 88 \delta\left|\lambda_{k_{i}}\right|\right)$ точка $z /|z|$ принадлежит $B\left(w_{2}, 3 \beta\right)$. Следовательно, с учетом (3.34) получаем

$$
\left|\sum \tilde{\psi}_{k_{j}}(z) e_{j}^{2}(z)\right| \leqslant C_{3} \exp \left(H_{L_{1}}(z)\right) \quad \forall z
$$

Функция $\nu_{2}(z)$ (определяемая, как и $\left.\nu_{1}(z)\right)$ отлична от нуля только на множествах $U_{p_{i}} \backslash V_{i}, i=1,2, \ldots$ Поэтому в силу $(3.27),(3.36)$ и свойства $\left.5^{\prime \prime}\right)$ для $e_{i}^{2}$ с учетом свойств компакта $L$ имеем оценку

$$
\left|\nu_{2}(z)\right| \leqslant \widetilde{C}_{9} C_{3} \exp \left(H_{L}(z)\right) \quad \forall z .
$$

Поскольку $L_{1}$ и $K+L$ - компакты в $D$, то найдется $\varepsilon_{2}>0$ такое, что

$$
H_{L_{1}}(z)+\varepsilon_{2}|z|<H_{D}(z), \quad H_{L}(z)+H_{K}(z)+5 \varepsilon_{2}|z|<H_{D}(z), \quad z \neq 0 .
$$

Согласно полученной оценке на $\nu_{2}(z)$ существует функция $\theta_{2}$, которая удовлетворяет уравнению $d \theta_{2}(z) / d \bar{z}=\nu_{2}(z)$ и неравенству

$$
\int_{\mathbb{C}}\left|\theta_{2}(z)\right|^{2} \exp \left(H_{L}(z)-4 \varepsilon_{1}|z|\right) d \lambda(z)<\infty
$$


Тогда, как и вьше, для $f_{2}$ (определяемой, как и $f_{1}$ ) получаем оценку

$$
h_{f_{2}}(z) \leqslant \max \left\{H_{L_{1}}(z)+\varepsilon_{2}|z|, H_{K}(z)+H_{L}(z)+5 \varepsilon_{2}|z|\right\}<H_{D}(z), \quad z \neq 0 .
$$

Следовательно, $f_{2} \in \mathbf{P}_{D}$.

3.3. Множество $\mathbf{S} \backslash\left(B\left(w_{1}, \beta_{1}\right) \cup B\left(w_{2}, \beta_{2}\right)\right)$ состоит из двух дуг $F_{1}$ и $F_{2}$ окружности $\mathbf{S}$, причем в окрестности $F_{1}$ функция $H_{D}$ конечна, а в окрестности $F_{2}$ бесконечна. Построим функцию $f_{3} \in \mathbf{P}_{D}$, которая вместе со своими производными принимает значения $b_{k, n}^{3}$ в точках $\lambda_{k}$, где $b_{k, n}^{3}=b_{k, n}$, если $\lambda_{k} \in \Gamma_{3}=\left\{t z: z \in F_{1}\right.$, $\left.t>T_{3}\right\}$, и $b_{k, n}^{3}=0$, если $\lambda_{k} \notin \Gamma_{3}, n=\overline{1, m_{k}}$. Фиксируем $\varepsilon \in\left(0, \alpha_{s} / 100\right)$, где $\alpha_{s}$ такое же, как и в (1.1). Поскольку $F_{1}-$ компакт в $\mathbf{S} \backslash \overline{J(D)}$ и $H_{D}$ непрерьвна на $\mathbf{S} \backslash \overline{J(D)}$ (в обычном смысле), то найдутся номер $m \geqslant s+1$ и $\alpha>0$ такие, что $H_{K_{m}}(z) \geqslant H_{D}(z)-\varepsilon, z \in E(6 \alpha)\left(E(\alpha)\right.$ - окрестность $\left.F_{1}\right)$. Пусть $h(z)=H_{K_{m}}(z)$ и $H(z)=H_{K_{m}}(z)+2 \varepsilon|z|$. По функциям $h(z), H(z)$ и числам $\varepsilon, \alpha$ найдем $\delta_{0}>0$ из леммы 3.3 и фиксируем положительное $\delta<\min \left\{\delta_{0}, \alpha / 22, \tilde{\delta} / 88\right\} \quad(\tilde{\delta}>0$ такое же, как и в $(3.28)$, где в качестве $F$ берем $\left.F_{1}\right)$. Сразу же наложим дополнительное условие на $\delta$ : при $\tau=88 \delta$ для функций $43 \varepsilon|z|$ и $44 \varepsilon|z|$ выполнено (3.13). Для выбранных $\delta, m$ и $F_{1}$ согласно условию 5$)$ теоремы найдем функцию $\varphi \in I_{\Lambda}(D)$. В силу условия 5) каждая точка $\lambda_{k}$ такая, что $\lambda_{k} /\left|\lambda_{k}\right| \in F_{1}$ и $\left|\lambda_{k}\right|>T$, принадлежит кругу $B\left(z_{k}, \delta\left|z_{k}\right|\right)$ для некоторого $z_{k} \in R_{m}$. Дальнейшее построение практически полностью совпадает с построением, проведенным в п. 3.1, с единственным исключением: в отличие от $\Gamma_{1}$ для определения $\Gamma_{3}$ не нужно подбирать число, подобное $\beta_{1}$.

3.4. Построим, наконец, функцию $f_{4} \in \mathbf{P}_{D}$, которая вместе со своими производными принимает значения $b_{k, n}^{4}$ в точках $\lambda_{k}$, где $b_{k, n}^{4}=b_{k, n}, n=\overline{1, m_{k}}$, если $\lambda_{k} \notin \Gamma_{1} \cup \Gamma_{2} \cup \Gamma_{3}$, и $b_{k, n}^{2}=0, n=\overline{1, m_{k}}$, если $\lambda_{k} \in \Gamma_{1} \cup \Gamma_{2} \cup \Gamma_{3}$.

Пусть $\varphi$ - произвольная функция из $I_{\Lambda}(D)$, не равная тождественно нулю (по условию $\left.I_{\Lambda}(D) \neq\{0\}\right)$. По теореме об оценке снизу на окружностях модуля целой функции конечного порядка и типа (см. [48, гл. I, §4, теорема 4.3]) для каждого $\delta>0$ существуют $a_{0}(\delta)>0$ и $r_{j}>0, j \geqslant 1$, такие, что $r_{j} \rightarrow+\infty, r_{j+1}<(1+\delta) r_{j}$ и выполнено

$$
\ln |\varphi(z)| \geqslant-a_{0}(\delta)|z|, \quad|z|=r_{j}, \quad j=1,2, \ldots
$$

Положим $R=\left\{z:|z|=r_{j}, j=1,2, \ldots\right\}$. Тогда для некоторого $T^{\prime}>0$ каждая точка $\lambda_{k}$ такая, что $\left|\lambda_{k}\right|>T$, принадлежит $R^{\delta}$. Таким образом, мы получили условие, подобное условию 5$)$ теоремы, но с более слабыми оценками.

Пусть $\varepsilon, \alpha=1, h(z)=a_{0}(\delta)|z|$ и $H(z)=d_{0}|z|$, где $d_{0}=\max _{y \in \mathrm{S}} h_{\varphi}(y)$. Согласно замечанию 3.4 найдем число $\delta_{0}>0$ из леммы 3.3 , не зависящее от $h$ и $H$. Поскольку $F_{2}$ - компакт в int $J(D)$, то существует его окрестность $E(3 \beta)$, в которой $H_{D}(z)=+\infty$. Кроме того, уменьшая при необходимости $\beta>0$, можно считать, что $3 \beta$ - число, которое определяется только по $F_{2}$ в лемме 3.6. Фиксируем положительное $\delta<\min \left\{\delta_{0}, \beta / 88, \tilde{\delta} / 88\right\} \quad(\tilde{\delta}>0$ такое же, как и в $(3.27))$. С учетом (3.37) и замечания 3.4 найдем множества $U_{p}$ и $T>T^{\prime}$, обладающие свойствами 1)-3) леммы 3.3 , и $\tilde{a}$ такое, что $\ln |\varphi(z)| \geqslant \tilde{a}|z|, z \in \partial U_{p}$. Далее, как и выше, применяем лемму 3.5 и выделяем $U_{p_{i}}, i \geqslant 1$, которые содержат все $\lambda_{k}$ из множества $\Gamma_{4}=\left\{t z: z \in F_{2}, t>T\right\}$. Имеется лишь конечное число точек $\lambda_{k}$, не попавших 
в объединение $\Gamma_{1} \cup \Gamma_{2} \cup \Gamma_{3} \cup \Gamma_{4}$. Выберем попарно непересекающиеся окрестности таких точек, которые к тому же не пересекаются ни с одним из $U_{p_{i}}$. Добавим эти окрестности к $U_{p_{i}}$ и после перенумерации будем считать, что сами $U_{p_{i}}, i \geqslant 1$, содержат все точки $\lambda_{k}$, не принадлежашие объединению $\Gamma_{1} \cup \Gamma_{2} \cup \Gamma_{3}$. При этом необходимые оценки на $\ln |\varphi|$ в окрестностях множеств $\partial U_{p_{i}}$ изменятся лишь на константу. Дальнейшее построение $f_{4}$ вполне аналогично построению $f_{2}$. Заметим только, что при выборе функций, подобных $e_{i}^{2}$, в свойстве 5) вместо (3.13) нужно использовать неравенства из замечания 3.4. Положим $f=f_{1}+f_{2}+f_{3}+f_{4}$. По построению $f$ - искомая функция. Теорема доказана.

ЗАмЕчАниЕ 3.11. Из доказательства теоремы 3.7 видно, что утверждение (i) является следствием только лишь условий 4) и 5).

\section{§4. Необходимые условия интерполяции и фундаментального принципа}

Здесь мы найдем необходимые условия интерполящии, которые, на первый взгляд, будут выглядеть даже сильнее достаточных. Прежде всего, докажем, что условия 1) и 2) теоремы 3.7 являются следствием разрешимости интерполяционной задачи.

ТЕОРема 4.1. Пусть $D$ - выпуклая область $и \Lambda=\left\{\lambda_{k}, m_{k}\right\}$. Если верно вложение $\Phi \subset \Sigma\left(\mathbf{P}_{D} \backslash I_{\Lambda}(D)\right)$, то:

(i) $S_{\Lambda}(D)=0$

(ii) $S_{\Lambda} \geqslant a>-\infty$.

ДокАЗАТЕЛЬСтво. (i) Как мы уже отмечали, для любой последовательности $\tilde{\Lambda}$ величина $S_{\tilde{\Lambda}}$ неположительна. Следовательно, $S_{\Lambda}(D) \leqslant 0$. Предположим, что для некоторого $\tilde{\varepsilon}>0$ верно неравенство $S_{\Lambda}(D)<-2 \tilde{\varepsilon}$. Тогда $S_{\Lambda}(F)<-\tilde{\varepsilon}$ для некоторого компакта $F \subset \mathbf{S} \backslash J(D)$, а значит, $S_{\Lambda(E(\beta))}<-\tilde{\varepsilon}$ для любой окрестности $E(\beta)$ этого компакта. Отсюда с учетом (3.1) следует, что для каждого $j \geqslant 4$ найдется точка $\lambda_{k_{j}}$ со свойствами: $\lambda_{k_{j}} /\left|\lambda_{k_{j}}\right| \in E(1 / k),\left|\lambda_{k_{j}}\right|>k$ и

$$
\left|\lambda_{k_{j}}\right|^{-1} \ln \left|q_{\Lambda}^{k_{j}}\left(\lambda_{k_{j}}, \lambda_{k_{j}}, 1 / j\right)\right|<-\tilde{\varepsilon} .
$$

Переходя к подпоследовательности, можно считать, что $\lambda_{k_{j}} /\left|\lambda_{k_{j}}\right|$ сходится к точке $x_{0} \in F$ и $\left|\lambda_{k_{j+1}}\right| /\left|\lambda_{k_{j}}\right|>2, j \geqslant 4$. Последнее означает, что для всех $\delta \in(0,1 / 2)$ круг $B\left(\lambda_{k_{j}}, \delta\left|\lambda_{k_{j}}\right|\right)$ не содержит точек $\lambda_{k_{p}}$ при $p \neq j$.

Пусть $\varepsilon \in(0, \tilde{\varepsilon} / 4)$. Выберем номер $m$ так, что $H_{K_{m}}\left(x_{0}\right) \geqslant H_{D}\left(x_{0}\right)-\varepsilon$. Рассмотрим последовательность $b=\left\{b_{k, n}\right\}_{k=1, n=1}^{\infty, m_{k}}$, где $b_{k_{j}, 1}=\exp H_{K_{m}}\left(\lambda_{k_{j}}\right)$ и $b_{k, n}=0$ для всех остальных $k, n$. Тогда $b \in \Phi$. Поэтому согласно условию теоремы существует функция $f \in \mathbf{P}_{D}$ такая, что $\widetilde{\Sigma}(f)=b$, т.е. $f^{(n-1)}\left(\lambda_{k}\right)=b_{k, n}, k \geqslant 1$, $n=\overline{1, m_{k}}$. В силу непрерывности $H_{K_{m}}, h_{f}$, выбора номера $m$ и неравенства $h_{f}(z)<H_{D}(z), z \neq 0$, для некоторого $\delta \in(0,1 / 6)$ имеем

$$
h_{f}(z) \leqslant H_{D}\left(x_{0}\right) \leqslant H_{K_{m}}(z)+2 \varepsilon, \quad z \in B\left(x_{0}, 6 \delta\right) .
$$

Отсюда, как и в $(3.11)$, получаем

$$
\ln |f(z)| \leqslant h_{f}(z)+\varepsilon|z| \leqslant H_{K_{m}}(z)+3 \varepsilon|z| \quad \forall z: z /|z| \in B\left(x_{0}, 6 \delta\right), \quad|z| \geqslant T .
$$


Поскольку $\lambda_{k_{j}} /\left|\lambda_{k_{j}}\right| \rightarrow x_{0}$, то в силу (3.13) (при малом $\delta$ ) верно неравенство

$$
\ln |f(z)| \leqslant H_{K_{m}}\left(\lambda_{k_{j}}\right)+4 \varepsilon\left|\lambda_{k_{j}}\right|, \quad z \in \overline{B\left(\lambda_{k_{j}}, 5 \delta\left|\lambda_{k_{j}}\right|\right)}, \quad j \geqslant j_{0} .
$$

Нетрудно заметить, что $\left|q_{\Lambda}^{k_{j}}\left(z, \lambda_{k_{j}}, \delta\right)\right| \geqslant 1, z \in \partial B\left(\lambda_{k_{j}}, 5 \delta\left|\lambda_{k_{j}}\right|\right)$. Поэтому

$\ln \left|f(z)\left(q_{\Lambda}^{k_{j}}\left(z, \lambda_{k_{j}}, \delta\right)\right)^{-1}\right| \leqslant H_{K_{m}}\left(\lambda_{k_{j}}\right)+4 \varepsilon\left|\lambda_{k_{j}}\right|, \quad z \in \partial B\left(\lambda_{k_{j}}, 5 \delta\left|\lambda_{k_{j}}\right|\right), \quad j \geqslant j_{0}$.

По построению $f(z)$ обрашается в нуль в точках $\lambda_{k} \in B\left(\lambda_{k_{j}}, \delta\left|\lambda_{k_{j}}\right|\right), k \neq k_{j}$, с кратностью, не меньшей, чем $m_{k}$. Следовательно, $f(z)\left(q_{\Lambda}^{k_{j}}\left(z, \lambda_{k_{j}}, \delta\right)\right)^{-1}$ - целая функция. Тогда по принципу максимума из предыдушего получаем

$$
\ln \left|f\left(\lambda_{k_{j}}\right)\left(q_{\Lambda}^{k_{j}}\left(\lambda_{k_{j}}, \lambda_{k_{j}}, \delta\right)\right)^{-1}\right| \leqslant H_{K_{m}}\left(\lambda_{k_{j}}\right)+4 \varepsilon\left|\lambda_{k_{j}}\right| .
$$

Отсюда с учетом (4.1), (3.1) и выбора числа $\varepsilon$ имеем

$$
\ln \left|f\left(\lambda_{k_{j}}\right)\right|<H_{K_{m}}\left(\lambda_{k_{j}}\right)+4 \varepsilon\left|\lambda_{k_{j}}\right|-\tilde{\varepsilon}\left|\lambda_{k_{j}}\right|<H_{K_{m}}\left(\lambda_{k_{j}}\right), \quad j \geqslant j_{1} .
$$

Это противоречит равенству $f\left(\lambda_{k_{j}}\right)=\exp \left(H_{K_{m}}\left(\lambda_{k_{j}}\right)\right), j \geqslant 4$. Таким образом, утверждение (i) доказано.

(ii) Утверждение содержательно лишь для неограниченной области, поскольку для ограниченной области $D$ оно является следствием утверждения (i). Доказательство утверждения (ii) аналогично проведенному вьше. Заметим лишь, что при оценке сверху $\ln |f(z)|$ вместо функции $H_{K_{m}}(z)+3 \varepsilon|z|$ необходимо взять $(a+1)|z|$, где $a=\sup \left\{h_{f}(z), z \in \mathbf{S}\right\}$, и затем воспользоваться соотношением $\left|\lambda_{k_{j}}\right|^{-1} \times$ $\ln \left|q_{\Lambda}^{k_{j}}\left(\lambda_{k_{j}}, \lambda_{k_{j}}, 1 / j\right)\right| \rightarrow-\infty, j \rightarrow \infty$, которое будет иметь место, если предположить, что $S_{\Lambda}=-\infty$. При выборе $f$ компакт $K_{m}$ можно заменить любым компактом из $D$. Теорема доказана.

Далее мы хотим доказать необходимость условий 3)-5) теоремы 3.7 (и даже более сильных условий) для разрешимости интерполяционной задачи при дополнительном предположении $m_{D}(\Lambda)=0$. Но вначале докажем необходимость более слабых условий. Введем функции

$$
\Psi_{j}(z)=\varlimsup_{w \rightarrow z} \sup \left\{\ln |\varphi(w)|: \varphi \in I_{\Lambda}(D),\|\varphi\|_{j} \leqslant 1\right\}, \quad j=1,2, \ldots
$$

Функция $\Psi_{j}$ является субгармонической как регуляризация верхней огибающей семейства субгармонических функций, равномерно ограниченных на каждом компакте (см., например, $[52$, гл. I, $\S 5$, п. 1$]$ ). Ясно, что $\left\{\Psi_{j}\right\}$ - возрастаюшая последовательность.

ТЕОРема 4.2. Пусть $D$ - выпуклая область и $\Lambda=\left\{\lambda_{k}, m_{k}\right\}$ такая, что $m_{D}(\Lambda)=0$. Предположим, ито $\Sigma: \mathbf{P}_{D} / I_{\Lambda}(D) \rightarrow \Phi-$ изоморфизм. Тогда для функиий $\Psi_{j}, \quad j=1,2, \ldots$, верны следующие утверждения:

(i) для каждого $m \geqslant 1$ и каждого компакта $F \subset \mathbf{S} \backslash J(D)$ существует $j \geqslant 1$ такое, что для любого $\delta>0$ найдутся $\beta, T>0$, удовлетворяющие условию: $\lambda_{k} \in R_{m, j}^{\delta}$, если $\lambda_{k} /\left|\lambda_{k}\right| \in E(\beta) u\left|\lambda_{k}\right|>T$, где $R_{m, j}=\left\{z: \Psi_{j}(z) \geqslant H_{K_{m}}(z)\right\} u$ $E(\beta)$ - $\beta$-окрестность $F$;

(ii) существуют $j \geqslant 1$ и $a_{0} \in \mathbb{R}$ такие, что для любого $\delta>0$ найдется $T>0$, удовлетворяющее условию: $\lambda_{k} \in R_{0}^{\delta}$, если $\left|\lambda_{k}\right|>T$, где $R_{0}=\{z$ : $\left.\Psi_{j}(z) \geqslant a_{0}|z|\right\}$. 
ДокАЗАТЕЛЬСТво. (i) Предположим, что утверждение неверно. Тогда для некоторых $m \geqslant 1$, компакта $F \subset \mathbf{S} \backslash J(D)$ и каждого $j \geqslant 1$ существуют $\delta>0$ и подпоследовательность $\left\{\lambda_{k_{p}}\right\}$ (зависящие от $j$ ) такие, что

$$
\lambda_{k_{p}} /\left|\lambda_{k_{p}}\right| \in E(1 / p), \quad \lambda_{k_{p}} \notin R_{m, j}^{\delta}, \quad p=1,2, \ldots, \quad\left|\lambda_{k_{p}}\right| \rightarrow \infty .
$$

Фиксируем $\varepsilon \in\left(0, \alpha_{m} / 5\right)$, где $\alpha_{m}$ такое же, как в (1.1). По условию оператор $\Sigma$ - изоморфизм. Это означает, что для некоторых $j \geqslant 1$ и $C>0$

$$
\left\|\Sigma^{-1}(b)\right\|_{j} \leqslant C\|b\|_{m+1} \quad \forall b \in \Phi_{m+1} .
$$

Пусть $\delta>0$ и $\left\{\lambda_{k_{p}}\right\}$ соответствуют номеру $j+1$. Переходя к подпоследовательности, можно считать, что $\left|\lambda_{k_{p+1}}\right| \geqslant 2\left|\lambda_{k_{p}}\right|, p \geqslant 1$. Тогда $\sum_{p}\left|\lambda_{k_{p}}\right|^{-1}<\infty$ и функция $g(z)=\prod_{p=1}^{\infty}\left(1-z / \lambda_{k_{p}}\right)$ является целой и имеет экспоненциальный минимальный тип, т. е. $h_{g}(z) \equiv 0$ (см., например, [48, гл. I, теорема 3.9]). Кроме того, ее нули $\left\{\lambda_{k_{p}}\right\}$ образуют так называемое регулярное множество, и поэтому для некоторого $\widetilde{T}>0$ верна оценка (см., например, [48, гл. I, теорема 6.4])

$$
\ln |g(z)| \geqslant-\varepsilon|z|, \quad|z| \geqslant \widetilde{T}, \quad z \notin B\left(\lambda_{k_{p}}, 1\right), \quad p=1,2, \ldots .
$$

Рассмотрим последовательность $b=\left\{b_{k, n}\right\}_{k=1, n=1}^{\infty, m_{k}}$, где $b_{k, n}=\exp \left(H_{K_{m+1}}\left(\lambda_{k_{p}}\right)\right)$, если $k=k_{p}, n=m_{k_{p}}$, и $b_{k, n}=0$ для всех остальных $k, n$. Тогда $b \in \Phi_{m+1}$ и $\|b\|_{m+1}=1$. Поэтому согласно условию теоремы с учетом (4.2) существует функция $f \in \mathbf{P}_{j}$ такая, что $\|f\|_{j} \leqslant 2 C$ и $f^{(n-1)}\left(\lambda_{k}\right)=b_{k, n}, k \geqslant 1, n=\overline{1, m_{k}}$. Пусть $\beta \in(0,1 / 2), \beta<\delta / 2$. Функция $f$ обрашается в нуль в точке $\lambda_{k_{p}}$ с кратностью $m(p)=m_{k_{p}}-1$. Поэтому $f(z)=\left(\left(z-\lambda_{k_{p}}\right) /\left(\beta\left|\lambda_{k_{p}}\right|\right)\right)^{m(p)} \tilde{f}(z)$, где $\tilde{f}$ целая. Отсюда имеем

$$
\exp \left(H_{K_{m+1}}\left(\lambda_{k_{p}}\right)\right)=f^{(m(p))}\left(\lambda_{k_{p}}\right)=m(p) ! \tilde{f}\left(\lambda_{k_{p}}\right)\left(\beta\left|\lambda_{k_{p}}\right|\right)^{-m(p)}
$$

Из условия $m_{D}(\Lambda)=0$ следует, что $m(p) /\left|\lambda_{k_{p}}\right| \rightarrow 0$. Последнее, как и в $(2.10)$, влечет за собой оценки

$$
\left|\lambda_{k_{p}}\right|^{m(p)} / m(p) ! \geqslant \exp \left(-\varepsilon\left|\lambda_{k_{p}}\right|\right), \quad \beta^{m(p)} \geqslant \exp \left(-\varepsilon\left|\lambda_{k_{p}}\right|\right)
$$

при $p \geqslant p_{0}$. Следовательно, из предыдушего получаем

$$
\left|\tilde{f}\left(\lambda_{k_{p}}\right)\right| \geqslant \exp \left(H_{K_{m+1}}\left(\lambda_{k_{p}}\right)-2 \varepsilon\left|\lambda_{k_{p}}\right|\right), \quad p \geqslant p_{0}
$$

По принципу максимума найдется $y_{p}$ такое, что $\left|y_{p}-\lambda_{k_{p}}\right|=\beta\left|\lambda_{k_{p}}\right|$ и

$$
\left|\tilde{f}\left(y_{p}\right)\right| \geqslant \exp \left(H_{K_{m+1}}\left(\lambda_{k_{p}}\right)-2 \varepsilon\left|\lambda_{k_{p}}\right|\right), \quad p \geqslant p_{0} .
$$

Отсюда с учетом (3.13) (при малом $\beta$ ) имеем

$$
\left|\tilde{f}\left(y_{p}\right)\right| \geqslant \exp \left(H_{K_{m+1}}\left(y_{p}\right)-3 \varepsilon\left|y_{p}\right|\right), \quad p \geqslant p_{0} .
$$


Тогда в силу выбора $y_{p}$

$$
\left|f\left(y_{p}\right)\right|=\left|\tilde{f}\left(y_{p}\right)\right| \geqslant \exp \left(H_{K_{m+1}}\left(y_{p}\right)-3 \varepsilon\left|y_{p}\right|\right), \quad p \geqslant p_{0} .
$$

Поскольку $h_{g}(z) \equiv 0$, то, как и в $(3.11)$, получаем оценку (где $\alpha_{j}-$ из $(1.1)$ )

$$
|g(z)| \leqslant C_{1} \exp \left(\alpha_{j}|z|\right) \quad \forall z .
$$

Рассмотрим функцию $\varphi(z)=\left(2 C C_{1}\right)^{-1} f(z) g(z)$. Из неравенств $(4.3),(4.4),(1.1)$ с учетом неравенства $\left|\lambda_{k_{p+1}}\right| \geqslant 2\left|\lambda_{k_{p}}\right|$ и выбора $y_{p}, \varepsilon$ следует, что

$$
\left|\varphi\left(y_{p}\right)\right| \geqslant \exp \left(H_{K_{m+1}}\left(y_{p}\right)-5 \varepsilon\left|y_{p}\right|\right) \geqslant \exp \left(H_{K_{m}}\left(y_{p}\right)\right), \quad p \geqslant p_{1} .
$$

С другой стороны, в силу $(4.5),(1.1)$ и выбора $f$ имеем

$$
|\varphi(z)| \leqslant\left(2 C C_{1}\right)^{-1} 2 C \exp \left(H_{K_{j}}(z)\right) C_{1} \exp \left(\alpha_{j}|z|\right) \leqslant \exp \left(H_{K_{j+1}}(z)\right) \quad \forall z
$$

т. е. $\|\varphi\|_{j+1} \leqslant 1$. По построению $\varphi$ обращается в нуль во всех точках $\lambda_{k}$ с кратностью, не меньшей, чем $m_{k}$. Поэтому $\varphi \in I_{\Lambda}(D)$. Тогда по определению $\Psi_{j+1} \mathrm{c}$ учетом (4.6) верно неравенство

$$
\Psi_{j+1}\left(y_{p}\right) \geqslant H_{K_{m}}\left(y_{p}\right), \quad p \geqslant p_{1}
$$

Таким образом, $y_{p} \in R_{m, j+1}, p \geqslant p_{1}$. В силу выбора $y_{p}$ и $\beta$ имеем

$$
\delta\left|y_{p}\right| \geqslant 2^{-1} \delta\left|\lambda_{k_{p}}\right|>\beta\left|\lambda_{k_{p}}\right|, \quad p \geqslant p_{1} .
$$

Это влечет за собой включение $\lambda_{k_{p}} \in B\left(y_{p}, \delta\left|y_{p}\right|\right) \subset R_{m, j+1}^{\delta}, p \geqslant p_{1}$, которое противоречит выбору $\left\{\lambda_{k_{p}}\right\}$. Утверждение (i) доказано.

(ii) Утверждение доказьвается аналогично. Заметим только, что при определении номера $j$ и последовательности $\left\{b_{k, n}\right\}$ достаточно взять $m=0$, а при оценке $\left|\tilde{f}\left(\lambda_{k_{p}}\right)\right|$ вместо (2.10) необходимо воспользоваться тем же неравенством, что и при получении (3.22). Кроме того, в этом случае $F$ совпадает с окружностью $\mathbf{S}$. Теорема доказана.

Покажем теперь, что выполнение для функций $\Psi_{j}$ свойств из утверждений (i), (ii) теоремы 4.2 влечет за собой существование функций $\varphi \in I_{\Lambda}(D)$ с аналогичными свойствами. Но прежде сделаем простое наблюдение. Пусть $\Lambda=\left\{\lambda_{k}, m_{k}\right\}-$ такая, что $I_{\Lambda}(\mathbb{C}) \neq\{0\}$. Введем функцию

$$
f_{\Lambda}(z)=\prod_{k \geqslant 1}\left(1-\frac{z}{\lambda_{k}}\right)^{m_{k}} \exp \left(\frac{m_{k} z}{\lambda_{k}}\right) .
$$

Поскольку точки $\lambda_{k}$ с кратностью $m_{k}, k \geqslant 1$, являются нулями целой функции экспоненциального типа $\varphi \in I_{\Lambda}(\mathbb{C})$, то (см., например, [48, гл. 1 , теоремы $2.2,3.1$ и 3.3]) $f_{\Lambda}$ - целая функция порядка не выше первого (возможно, бесконечного типа при порядке один), обрашаюшаяся в нуль лишь в точках $\lambda_{k} \mathrm{c}$ кратностью $m_{k}$, 
$k \geqslant 1$. В частности, это означает, что для некоторых $C, b>0$ выполнено неравенство $\left|f_{\Lambda}(z)\right| \leqslant C \exp \left(b|z|^{2}\right) \quad \forall z$. Тогда по теореме об оценке снизу на окружностях целой функции конечного порядка (см. [48, гл. I, $\S 4$, теорема 4.3]) существуют $a>0$ и последовательность $r_{j} \rightarrow+\infty$ такие, что

$$
r_{j+1}<2 r_{j}, \quad \ln \left|f_{\Lambda}(z)\right| \geqslant-a|z|^{2}, \quad|z|=r_{j}, \quad j \geqslant 1 .
$$

Пусть $d>0, f \in I_{\Lambda}(\mathbb{C})$ и $|\varphi(z)| \leqslant \exp (d|z|) \quad \forall z$. Функция $\varphi / f_{\Lambda}$ целая, и в силу (4.7) имеем

$$
\left|\frac{\varphi(z)}{f_{\Lambda}(z)}\right| \leqslant \exp \left(d|z|+a|z|^{2}\right), \quad|z|=r_{j}, \quad j \geqslant 1
$$

Отсюда по принципу максимума получаем

$$
\left|\frac{\varphi(z)}{f_{\Lambda}(z)}\right| \leqslant \exp \left((d+a) r_{j+1}^{2}\right) \leqslant \exp \left(4(d+a)|z|^{2}\right)
$$

где $|z| \geqslant 1, r_{j} \leqslant|z| \leqslant r_{j+1}, j \geqslant 1$. Следовательно,

$$
\left|\frac{\varphi(z)}{f_{\Lambda}(z)}\right| \leqslant C \exp \left(4(d+a)|z|^{2}\right) \quad \forall z
$$

и $C>0$ зависит от $f_{\Lambda}$ и $d$, но не зависит от $f$. Положим

$$
\psi(z)=\varlimsup_{w \rightarrow z} \sup \left\{\ln |\varphi(w)|-\ln \left|f_{\Lambda}(w)\right|: f \in I_{\Lambda}(\mathbb{C}),|\varphi(w)| \leqslant \exp H_{L}(z)\right\},
$$

где $L$ - ограниченное выпуклое множество. Тогда, как и $\Psi_{j}$, функция $\psi$ субгармоническая. Кроме того, верна оценка

$$
\psi(z) \leqslant \widetilde{C}+\tilde{a}|z|^{2} \quad \forall z
$$

Теорема 4.3. Пусть $D$ - выпуклая область и $\Lambda=\left\{\lambda_{k}, m_{k}\right\}$. Предположим, что для функций $\Psi_{j}, j \geqslant 1$, выполнены утверждения (i) и (ii) теоремы 4.2. Тогда верны и следующие утверждения:

(i) для каждого $p \geqslant 1$ и каждого компакта $F \subset \mathbf{S} \backslash J(D)$ существует функция $\varphi \in I_{\Lambda}(D)$ такая, что для любого $\tilde{\delta}>0$ найдутся $\tilde{\beta}, \widetilde{T}>0$, удовлетворяющие условию: $\lambda_{k} \in R_{p}^{\tilde{\delta}}$, если $\lambda_{k} /\left|\lambda_{k}\right| \in E(\tilde{\beta}) u\left|\lambda_{k}\right|>\widetilde{T}$, где $R_{p}=\left\{z: \ln |\varphi(z)| \geqslant H_{K_{p}}(z)\right\}$ и $E(\tilde{\beta})$ - $\beta$-окрестность $F ;$

(ii) существуют $\varphi \in I_{\Lambda}(D)$ и $a_{0} \in \mathbb{R}$ такие, что для любого $\tilde{\delta}>0$ найдется $\widetilde{T}>0$, удовлетворяющее условию: $\lambda_{k} \in R_{0}^{\delta}$, если $\left|\lambda_{k}\right|>\widetilde{T}$, где $R_{0}=\{z$ : $\left.\ln |\varphi(z)| \geqslant a_{0}(z)\right\}$. 
ДокаЗАТЕЛЬСТво. (i) Фиксируем $p \geqslant 1$ и компакт $F \subset \mathbf{S} \backslash J(D)$. Пусть $\varepsilon \in$ $\left(0, \alpha_{p} / 31\right)$, где $\alpha_{p}$ - такое же, как и в (1.1). Поскольку $F$ - компакт и (как мы отмечали выше) сужение $H_{D}$ на $F$ непрерывно, то найдется номер $m \geqslant p+1$, для которого выполнено неравенство

$$
H_{K_{m}}(z) \geqslant H_{D}(z)-\varepsilon, \quad z \in F \text {. }
$$

По $m$ и $F$ согласно утверждению (i) теоремы 4.2 найдем $j \geqslant 1$. Функция

$$
\widetilde{\Psi}_{j}(z)=\varlimsup_{w \rightarrow z} \sup \left\{\ln |\varphi(w)|-\ln \left|f_{\Lambda}(z)\right|: \varphi \in I_{\Lambda}(D),\|\varphi\|_{j} \leqslant 1\right\}
$$

субгармонична во всей плоскости и удовлетворяет оценке вида (4.8). Тогда в силу $[53$, теорема 5$]$ найдутся целая функция $\varphi_{j}(z)$, постоянная $C>0$ и исключительное множество $E \subset \mathbb{C}$ такие, что

$$
|\ln | \varphi_{j}(z)\left|-\widetilde{\Psi}_{j}(z)\right| \leqslant C \ln |z|, \quad z \in \mathbb{C} \backslash E,
$$

причем $E$ может быть покрыто кружками $B_{i}=B\left(z_{i}, r_{i}\right), i \geqslant 1$, такими, что $\sum r_{i}=A<\infty$. Положим $\varphi(z)=\varphi_{j}(z) f_{\Lambda}(z)$. Тогда $\varphi$ - целая функция. Покажем, что она искомая. Прежде всего заметим, что $\varphi$ обрашается в нуль в точках $\lambda_{k} \mathrm{c}$ кратностью, не меньшей, чем $m_{k}$. Далее, функция $\ln \left|f_{\Lambda}\right|$ непрерьвна (в широком смысле) в каждой точке плоскости. Поэтому с учетом определения $\Psi_{j}, \widetilde{\Psi}_{j}$ верно равенство $\Psi_{j}(z)=\widetilde{\Psi}_{j}(z)+\ln \left|f_{\Lambda}(z)\right|$. Отсюда и из (4.10)

$$
|\ln | \varphi(z)\left|-\Psi_{j}(z)\right|=|\ln | \varphi_{j}(z)\left|-\widetilde{\Psi}_{j}(z)\right| \leqslant C \ln |z|, \quad z \in \mathbb{C} \backslash E .
$$

Пусть $\tilde{\varepsilon}>0$. Тогда, учитывая определение $\Psi_{j}$, для некоторого $t(\tilde{\varepsilon})>0$ получаем неравенство

$$
\ln |\varphi(z)| \leqslant \Psi_{j}(z)+C \ln |z| \leqslant H_{K_{j}}(z)+\tilde{\varepsilon}|z|, \quad z \in \mathbb{C} \backslash E, \quad|z| \geqslant t(\tilde{\varepsilon}) .
$$

Пусть $|z|>3 A+t(\tilde{\varepsilon})$. Поскольку сумма диаметров кружков $B_{i}$ равна $2 A$, то в круге $B(z, 3 A)$ найдется окружность, на которой вьполнено (4.12). Тогда

$$
\ln |\varphi(z)| \leqslant \sup _{y \in B(z, 3 A)}\left(H_{K_{j}}(y)+\tilde{\varepsilon}|y|\right) .
$$

Используя теперь (3.13), найдем $t_{1}(\tilde{\varepsilon})>0$ такое, что

$$
\ln |\varphi(z)| \leqslant H_{K_{j}}(z)+2 \tilde{\varepsilon}|z|, \quad|z| \geqslant t_{1}(\tilde{\varepsilon}) .
$$

Отсюда следует неравенство $h_{\varphi}(z) \leqslant H_{K_{j}}(z)+2 \tilde{\varepsilon}|z|$, а в силу произвольности $\tilde{\varepsilon}>0$ и неравенство $h_{\varphi}(z) \leqslant H_{K_{j}}(z) \forall z$. Таким образом, $\varphi \in \mathbf{P}_{D}$, а значит, $\varphi \in I_{\Lambda}(D)$. Пусть $\tilde{\delta}>0$. Используя $(4.9)$, непрерьвность $H_{K_{m}}, H_{K_{j}}$ и то, что $H_{K_{j}}(z)<H_{D}(z), z \neq 0$, найдем $\alpha \in(0,1 / 27), \alpha<\tilde{\delta} / 6$, такое, что

$$
2 \varepsilon+H_{K_{m}}(z) \geqslant H_{K_{j}}(z), \quad z \in E(27 \alpha) .
$$


Кроме того, можно считать, что при $\tau=12 \alpha$ для $h_{0}(z)=H_{K_{m}}(z)+2 \varepsilon|z|$ и $h_{0}(z)=H_{K_{m}}(z)-28 \varepsilon|z|$ выполнено (3.13). Пусть $\delta \in(0, \alpha / 2)$. По $\delta$ согласно утверждению (i) теоремы 4.2 определим $\beta, T>0$. Фиксируем положительное $\tilde{\beta}<\min \{\alpha, \beta\}$ и $\widetilde{T}>\max \left\{T, 3 A \alpha^{-1}(1+\delta), 2(1-2 \alpha)^{-1}(1+\delta) T_{1}\right\}$, где $T_{1}>0$ выбрано так, что $C \ln |w| \leqslant \varepsilon|w|$ при $|w| \geqslant T_{1}$. Пусть $\lambda_{k}$ удовлетворяет условиям $\left|\lambda_{k}\right|>\widetilde{T}$ и $\lambda_{k} /\left|\lambda_{k}\right| \in E(\tilde{\beta})$. В силу утверждения (i) теоремы 4.2 для некоторого $z^{\prime} \in R_{m, j}$ круг $B\left(z^{\prime}, \delta\left|z^{\prime}\right|\right)$ содержит $\lambda_{k}$. Тогда $\left|z^{\prime}\right|>\max \left\{3 A \alpha^{-1}, 2(1-2 \alpha)^{-1} T_{1}\right\}$. По определению $\Psi_{j}$ и $R_{m, j}$ найдем точку $z$ и функцию $\tilde{\varphi} \in I_{\Lambda}(D)$ такие, что $|z|>\max \left\{2 A \alpha^{-1},(1-2 \alpha)^{-1} T_{1}\right\}, \quad \lambda_{k} \in B(z, 2 \delta|z|), \ln |\tilde{\varphi}(y)| \leqslant H_{K_{j}}(y) \quad \forall y$ и $\ln |\tilde{\varphi}(z)| \geqslant H_{K_{m}}(z)-\varepsilon|z|$. Нетрудно видеть, что $z /|z| \in E(3 \alpha)$. Поэтому в силу (4.13) и (3.13) получаем

$$
\ln |\tilde{\varphi}(y)| \leqslant H_{K_{m}}(z)+3 \varepsilon|z|, \quad y \in B(z, 12 \alpha|z|) .
$$

Рассмотрим функцию $\varphi_{z}(y)=\tilde{\varphi}(y) / \tilde{\varphi}(z)$. Она обладает следующими свойствами: $\varphi_{z}(z)=1$ и $\ln \left|\varphi_{z}(y)\right| \leqslant 4 \varepsilon|z|, y \in B(z, 12 \alpha|z|)$. Тогда по теореме об оценке снизу модуля аналитической функции (см., например, [48, гл. $1, \S 4$, теорема 4.2]) имеет место неравенство

$$
\ln \left|\varphi_{z}(y)\right| \geqslant-(3+\ln 24)(4 \varepsilon|z|) \geqslant-28 \varepsilon|z|,
$$

которое выполнено в $B(z, 2 \alpha|z|)$, но вне исключительных кружков $\widetilde{B}_{s}$ с суммой радиусов, равной $\alpha|z| / 2$. Поскольку $\alpha|z|>2 A$, то сумма диаметров всех кружков $\widetilde{B}_{s}$ и $B_{i}$ строго меньше $2 \alpha|z|$. Следовательно, в круге $B(z, 2 \alpha|z|)$ найдется окружность, на которой выполнено (4.14) и (4.11). Пусть $w$ - произвольная точка этой окружности. Тогда из неравенства (4.14), оценки снизу на $|\tilde{\varphi}(z)|$ и (3.13) следует неравенство $\ln |\tilde{\varphi}(w)| \geqslant H_{K_{m}}(w)-30 \varepsilon|w|$. Отсюда с учетом определения $\Psi_{j},(4.11)$ и выбора $\widetilde{T}$ получаем

$$
\ln |\varphi(w)| \geqslant \Psi_{j}(w)-\varepsilon|w| \geqslant \ln |\tilde{\varphi}(w)|-\varepsilon|w| \geqslant H_{K_{m}}(w)-31 \varepsilon|w| .
$$

Учитывая еше выбор номера $m$ и числа $\varepsilon>0$, а также неравенство (1.1), имеем

$$
\ln |\varphi(w)| \geqslant H_{K_{m}}(w)-31 \varepsilon|w| \geqslant H_{K_{p+1}}(w)-\alpha_{p}|w| \geqslant H_{K_{p}}(w) .
$$

Таким образом, $w \in R_{p}$. Далее, поскольку $w \in B(z, 2 \alpha|z|)$, а в силу выбора $\delta$ $\lambda_{k} \in B(z, 2 \delta|z|) \subset B(z, \alpha|z|)$, то в силу выбора $\alpha$ круг $B(w, \tilde{\delta}|w|)$ содержит $\lambda_{k}$. Следовательно, $\lambda_{k} \in R_{p}^{\tilde{\delta}}$. Утверждение (i) полностью доказано.

(ii) Доказательство утверждения проводится по упрошенной схеме доказательства утверждения (i).

ЗАмечАниЕ 4.4. В теоремах 4.2, 4.3 мы получили необходимые условия интерполяции, которые оказались сильнее достаточных условий 3)-5) теоремы 3.7. В частности, для разрешимости интерполяционной задачи необходимо существование некоторых оценок снизу на функции $\varphi \in I_{\Lambda}(D)$ в относительной близости от всех точек $\lambda_{k}$ (а не только от тех точек, которые лежат в окрестности множества, где $H_{D}$ конечна, как в теореме 3.7$)$. Это обстоятельство позволит нам ослабить ограничения, приведенные в теореме 3.7 , отказавшись от условия 3 ). Более точно, имеет место следующее утверждение. 
СЛЕДСТВИЕ 4.5. Пусть $\Lambda=\left\{\lambda_{k}, m_{k}\right\}$ - такая, что $I_{\Lambda}(\mathbb{C}) \neq\{0\}$. Предnoложим, что $S_{\Lambda} \geqslant a>-\infty$. Тогда выполнено условие 3) теоремы 3.7 для любой выпуклой области $D$.

ДокАЗАТельство. Пусть вначале $D=\mathbb{C}$. Тогда, как было отмечено в замечании 3.10 , выполнены все условия теоремы 3.7 , а значит, и ее утверждения. Следовательно, оператор $\Sigma: \mathbf{P}_{\mathbb{C}} / I_{\Lambda}(\mathbb{C}) \rightarrow \Phi-$ изоморфизм. По теоремам 4.2 и 4.3 это влечет за собой существование функции $\tilde{\varphi} \in I_{\Lambda}(\mathbb{C})$ и $\tilde{\alpha}_{0} \in \mathbb{R}$ таких, что для любого $\tilde{\delta}>0$ найдется $\widetilde{T}>0$, удовлетворяющее условию: $\lambda_{k} \in R_{0}^{\tilde{\delta}}$, если $\left|\lambda_{k}\right|>\widetilde{T}$, где $R_{0}=\left\{z: \ln |\tilde{\varphi}(z)| \geqslant \tilde{a}_{0}|z|\right\}$.

Пусть теперь $D$ - произвольная вьпуклая область такая, что $J(D) \cap \partial J(D)$ не пусто (если $J(D) \cap \partial J(D)$ пусто, то условие 3 ) теоремы 3.7 выполнено тривиально). Тогда $D$ - неограниченная область, отличная от плоскости, полосы и полуплоскости. В этом случае множество $\mathbb{C} \backslash J(D)$ - угол с вершиной в нуле и раствора не больше $\pi$, а $J(D)$ - угол раствора не меньше $\pi$, содержаший хотя бы один свой граничный луч. Следовательно, в $J(D)$ лежит полуплоскость

$$
\Pi=\left\{t \exp (i \alpha): \alpha \in\left(\alpha_{0}-\pi / 2, \alpha_{0}+\pi / 2\right), t>0\right\}
$$

такая, что луч $\left\{t \exp \left(i\left(\alpha_{0}+\pi / 2\right)\right), t>0\right\}$ также лежит в $J(D)$.

Отметим, что для любого $y \in \mathbb{C}$ функция $\varphi(z)=\tilde{\varphi}(z) \exp (y z)$ обладает всеми свойствами из условия 3) теоремы 3.7 , за исключением, возможно, одного: $\varphi \in$ $I_{\Lambda}(D)$. Поскольку $\tilde{\varphi} \in I_{\Lambda}(\mathbb{C})$, то и это свойство будет иметь место, если $\varphi \in \mathbf{P}_{D}$. Таким образом, нам достаточно показать, что для некоторого $y$ верно включение $\varphi \in \mathbf{P}_{D}$. Последнее равносильно тому, что сдвиг сопряженной диаграммы $K$ функции $\tilde{\varphi}$ на вектор $y$ лежит в $D$. Действительно, неравенство

$$
h_{\varphi}(z)=h_{\tilde{\varphi}}(z)+\operatorname{Re}(z y)=H_{K}(z)+\operatorname{Re}(z y)<H_{D}(z), \quad z \neq 0,
$$

выполнено тогда и только тогда, когда $y+K \subset D$.

Пусть $d<\min _{z \in \mathrm{S}} H_{D}(z)$ и $d_{1} \geqslant \max _{z \in \mathrm{S}} H_{K}(z), d_{1}>d$. В силу непрерьвности $H_{D}$ (в широком смысле) в точке $\tilde{z}=\exp \left(i\left(\alpha_{0}+\pi / 2\right)\right)$ найдется $\gamma \in(0, \pi / 8)$ такое, что верна оценка

$$
H_{D}(\exp (i \alpha))>2 d_{1}-d, \quad \alpha \in\left[\alpha_{0}+\pi / 2, \alpha_{0}+2 \gamma+\pi / 2\right) .
$$

Положим $y(t)=t \exp \left(-i\left(\alpha_{0}+\gamma\right)\right)$. Выберем $t_{1}>0$ из условия

$$
\operatorname{Re}\left(y\left(t_{1}\right) \exp \left(i\left(\alpha_{0}+2 \gamma+\pi / 2\right)\right)\right)=-t_{1} \sin \gamma=d-d_{1} .
$$

Тогда

$$
\begin{array}{ll}
\operatorname{Re}\left(y\left(t_{1}\right) \exp (i \alpha)\right) \leqslant-t_{1} \sin \gamma=d-d_{1}, & \alpha \in\left[\alpha_{0}+2 \gamma+\pi / 2, \alpha_{0}+3 \pi / 2\right], \\
\operatorname{Re}\left(y\left(t_{1}\right) \exp (i \alpha)\right) \leqslant t_{1} \sin \gamma=d_{1}-d, & \alpha \in\left[\alpha_{0}+\pi / 2, \alpha_{0}+2 \gamma+\pi / 2\right) .
\end{array}
$$

Пусть $y=y\left(t_{1}\right)$. Из предыдущего получаем

$$
\begin{gathered}
H_{K}(\exp (i \alpha))+\operatorname{Re}(y \exp (i \alpha)) \leqslant d_{1}+d-d_{1}=d<H_{D}(\exp (i \alpha)), \\
\alpha \in\left[\alpha_{0}+2 \gamma+\pi / 2, \alpha_{0}+3 \pi / 2\right] ;
\end{gathered}
$$




$$
\begin{gathered}
H_{K}(\exp (i \alpha))+\operatorname{Re}(y \exp (i \alpha)) \leqslant d_{1}+d_{1}-d<H_{D}(\exp (i \alpha)), \\
\alpha \in\left[\alpha_{0}+\pi / 2, \alpha_{0}+2 \gamma+\pi / 2\right) \\
H_{K}(\exp (i \alpha))+\operatorname{Re}(y \exp (i \alpha))<H_{D}(\exp (i \alpha))=+\infty \\
\alpha \in\left(\alpha_{0}-\pi / 2, \alpha_{0}+\pi / 2\right) .
\end{gathered}
$$

Таким образом, $H_{K}(z)+\operatorname{Re}(z y)<H_{D}(z), z \neq 0$, т.е. $y+K \subset D$. Следствие полностью доказано.

Далее рассмотрим случай ограниченной вьпуклой области $D$. Здесь мы получим более простые по форме и более сильные условия, необходимые для разрешимости интерполящионной задачи.

Теорема 4.6. Пусть $D$ - ограниченная выпуклая область и $\Lambda=\left\{\lambda_{k}, m_{k}\right\}-$ такая, ито $m(\Lambda)=0$. Предположим, ито $\Sigma: \mathbf{P}_{D} / I_{\Lambda}(D) \rightarrow \Phi-$ изоморфизм. Тогда для каждого $m \geqslant 1$ существует $\varphi_{m} \in I_{\Lambda}(D)$ такая, что $\underline{h}_{\varphi_{m}} \geqslant H_{K_{m}}$.

ДокаЗАТеЛЬСТво. Пусть $m \geqslant 1$. Фиксируем $\varepsilon \in\left(0, \alpha_{m} / 30\right)$, где $\alpha_{m}$ - такое же, как и в (1.1). Выберем номер $j \geqslant m+1$, для которого верно неравенство

$$
H_{K_{j}}(z) \geqslant H_{D}(z)-\varepsilon, \quad z \in \mathbf{S} .
$$

Согласно теоремам 4.2 и 4.3 (где в качестве $F$ берем окружность $\mathbf{S}$ ) найдем функцию $\varphi \in I_{\Lambda}(D)$ такую, что для любого $s \geqslant 1$ существует $T_{s}>0$, удовлетворяющее следуюшему условию: $\lambda_{k} \in R_{j}^{1 / s}$, если $\left|\lambda_{k}\right|>T_{s}$. Положим $\alpha=1, R=R_{j}$, $h(z)=H_{K_{j}}(z)$ и $H(z)=H_{K_{j}}(z)+\varepsilon|z|$. С учетом (4.15) по $\varepsilon, \alpha, h, H$ и $R$ согласно лемме 3.3 определим $\delta_{0}, T>0$ и для каждого $\delta=1 / s<\delta_{0}$ найдем открытые множества $U_{p}=U_{p, s}, \quad p \geqslant 1$, обладающие свойствами 1)-4) этой леммы. По свойствам 3) и 4) каждое $\lambda_{k}$ такое, что $\left|\lambda_{k}\right| \geqslant \max \left\{T, T_{s}\right\}$, принадлежит одному из множеств $U_{p, s}$ (при фиксированном $s$ ). Таким образом, для некоторого $k_{0}$ каждое $\lambda_{k}, k>k_{0}$, принадлежит хотя бы одному из $U_{p, s}, p \geqslant 1, s>1 / \delta_{0}$.

Построим теперь множества $V_{i}, i=0,1, \ldots$ В качестве $V_{1}$ возьмем множество $U_{p, s}$ с максимальным номером $s$, содержащее $\lambda_{k_{0}+1}$ (такой номер найдется, поскольку в противном случае в силу свойств 1) и 4) леммы 3.3 в точке $\lambda_{k_{0}+1}$ будет выполнено неравенство из свойства 4$)$, что невозможно, так как $\left.\varphi\left(\lambda_{k}\right)=0, k \geqslant 1\right)$. Предположим, что мы уже построили $V_{1}, \ldots, V_{r}$, и пусть $\lambda_{k}$ - точка с минимальным номером $k>k_{0}$, не попавшая ни в одно из $V_{i}, i=1, \ldots, r$. Тогда в качестве $V_{r+1}$ возьмем $U_{p, s} \backslash \bigcup_{i=1}^{r} \overline{V_{i}}$, где $U_{p, s}$ - множество с максимальным номером $s$, содержащее $\lambda_{k}$. Наконец, в качестве $V_{0}$ возьмем любое открытое ограниченное множество, содержашее все $\lambda_{k}$, не попавшие ни в одно из $V_{i}$, и не пересекаюшееся ни с одним из $V_{i}, i \geqslant 1$. По построению $V_{i}, i=0,1, \ldots$, обладают следующими свойствами. Граница каждого $V_{i}, i \geqslant 1$, состоит из кусков гранищ множеств $U_{p, s}$. Поэтому по свойству 4) леммы 3.3 верна оценка

$$
\begin{aligned}
\ln |\varphi(z)| & \geqslant C+H_{K_{j}}(z)-2 \varepsilon|z|-(3+\ln 24)\left(H_{K_{j}}(z)+\varepsilon|z|-H_{K_{j}}(z)+3 \varepsilon|z|\right) \\
& \geqslant C+H_{K_{j}}(z)-30 \varepsilon|z| \geqslant C+H_{K_{m}}(z), \quad z \in \partial V_{i}, \quad i=0,1, \ldots
\end{aligned}
$$


В последнем неравенстве мы воспользовались (1.1) и выбором $\varepsilon, j$. Каждая точка $\lambda_{k}$ попадает в одно из $V_{i}, i \geqslant 0$. Пусть $\lambda_{k_{i}}$ одна из таких точек, попавшая в $V_{i}$. Тогда $k_{i} \rightarrow \infty$ при $i \rightarrow \infty$, и поэтому $\left|\lambda_{k_{i}}\right| \rightarrow \infty$ при $i \rightarrow \infty$. Если $\left|\lambda_{k_{i}}\right| \geqslant \max \left\{T, T_{s}\right\}$, то по свойству 1) леммы 3.3 имеет место вложение $V_{i} \subset B\left(y, 88 s^{-1}|y|\right)$ для каждого $y \in V_{i}$. Рассмотрим функцию

$$
\psi(z)= \begin{cases}\ln |\varphi(z)|-\ln \left|f_{\Lambda}(z)\right|, & z \in \cup \overline{V_{i}}, \\ \max \left\{\ln |\varphi(z)|-\ln \left|f_{\Lambda}(z)\right|, C+H_{K_{m}}(z)-\ln \left|f_{\Lambda}(z)\right|\right\}, & z \notin \cup \overline{V_{i}}\end{cases}
$$

На множестве $\cup V_{i}$ функция $\psi(z)$ совпадает с $\ln \left|\varphi(z) / f_{\Lambda}(z)\right|$, и поскольку $\varphi \in$ $I_{\Lambda}(D)$, то она субгармонична на $\cup V_{i}$. На дополнении к $\cup \overline{V_{i}}$ функция $f_{\Lambda}$ не имеет нулей, а потому $\ln \left|f_{\Lambda}\right|$ гармонична. Следовательно, $\psi(z)$ субгармонична на $\mathbb{C} \backslash \cup \overline{V_{i}}$ как максимум субгармонических. Пусть $z \in \partial V_{i}$. Тогда в силу (4.16) имеют место соотношения

$$
\psi(z)=\ln |\varphi(z)|-\ln \left|f_{\Lambda}(z)\right| \geqslant \varlimsup_{y \rightarrow z}\left(\ln |\varphi(y)|-\ln \left|f_{\Lambda}(y)\right|\right)=\varlimsup_{y \rightarrow z} \psi(y),
$$

т. е. $\psi(y)$ полунепрерьвна сверху в точке $z$. Кроме того, при $\tau>0$

$$
\begin{aligned}
\psi(z)=\ln |\varphi(z)|-\ln \left|f_{\Lambda}(z)\right| & \leqslant \frac{1}{\pi \tau^{2}} \int_{B(z, \tau)}\left(\ln |\varphi(y)|-\ln \left|f_{\Lambda}(y)\right|\right) d \lambda(y) \\
& \leqslant \frac{1}{\pi \tau^{2}} \int_{B(z, \tau)} \psi(y) d \lambda(y) .
\end{aligned}
$$

Таким образом, функция $\psi$ субгармонична во всей плоскости. Покажем, что она удовлетворяет оценке вида (4.8). Как мы уже показали ранее, функция $\ln |\varphi(z)|-$ $\ln \left|f_{\Lambda}(z)\right|$ удовлетворяет подобной оценке:

$$
\ln |\varphi(z)|-\ln \left|f_{\Lambda}(z)\right| \leqslant C_{1}+a_{1}|z|^{2} \quad \forall z .
$$

Отсюда и из (4.16) следует, что

$$
\ln \left|f_{\Lambda}(z)\right| \geqslant C_{2}+a_{2}|z|^{2}, \quad z \in \partial V_{i}, \quad i=0,1, \ldots
$$

Используя это неравенство и неравенство (4.7), по принципу максимума для субгармонических функций получаем

$$
H_{K_{m}}(z)-\ln \left|f_{\Lambda}(z)\right| \leqslant C_{3}+a_{3}|z|^{2}, \quad z \notin \cup V_{i} .
$$

Таким образом, $\psi$ удовлетворяет оценке вида (4.8). Тогда в силу [53, теорема 5$]$ найдутся такие целая функция $f_{m}(z)$, постоянная $\widetilde{C}>0$ и исключительное множество $E \subset \mathbb{C}$, что

$$
|\ln | f_{m}(z)|-\psi(z)| \leqslant \widetilde{C} \ln |z|, \quad z \in \mathbb{C} \backslash E,
$$

причем $E$ может быть покрыто кружками $B_{j}=B\left(z_{j}, r_{j}\right), j \geqslant 1$, такими, что $\sum r_{j}=A<\infty$. Положим $\varphi_{m}(z)=f_{m}(z) f_{\Lambda}(z)$. Пользуясь тем, что $\varphi \in \mathbf{P}_{s}$ для некоторого $s \geqslant 1$, как и в теореме 4.3 , показываем, что $\varphi_{m} \in I_{\Lambda}(D)$. 
Предположим, что необходимое неравенство не выполнено, т.е. $\underline{h}_{\varphi_{m}}(y)<$ $H_{K_{m}}(y)-3 \tilde{\varepsilon}$ для некоторых $\tilde{\varepsilon}>0$ и $y \in \mathbb{C}$. В силу однородности $\underline{h}_{\varphi_{m}}$ и $H_{K_{m}}$ можно считать, что $y \in \mathbf{S}$. Тогда согласно [54, предложение 9.3] существуют $\delta \in(0,1)$ и последовательность $\left\{t_{r}\right\}, t_{r} \rightarrow+\infty$, такие, что

$$
\ln \left|\varphi_{m}\left(t_{r} w\right)\right| / t_{r} \leqslant H_{K_{m}}(y)-2 \tilde{\varepsilon}, \quad w \in B(y, \delta), \quad r=1,2, \ldots
$$

Уменьшая при необходимости $\delta$, в силу (3.13) получаем

$$
\ln \left|\varphi_{m}(z)\right| \leqslant H_{K_{m}}(z)-\tilde{\varepsilon}|z|, \quad z \in B\left(t_{r} y, \delta t_{r}\right), \quad r=1,2, \ldots
$$

С другой стороны, из (4.16), (4.17) и определения $\psi(z)$ следует оценка

$$
\ln \left|\varphi_{m}(z)\right| \geqslant H_{K_{m}}(z)-\widetilde{C} \ln |z|, \quad z \notin \cup V_{i} \cup E .
$$

Если $\delta t_{r}>4 A$, то в кольце $B\left(t_{r} y, \delta t_{r}\right) \backslash B\left(t_{r} y, \delta t_{r} / 2\right)$ найдется окружность $S_{r}$, не пересекающаяся с $E$. Поэтому для некоторого $r_{0}$ и всех $r \geqslant r_{0}$ множество $\cup V_{i}$ содержит $S_{r}$ (в противном случае (4.18) противоречит (4.19)). Поскольку $V_{i}$, $i \geqslant 1$, открыты и попарно не пересекаются, каждая окружность $S_{r}$ при $r \geqslant r_{0}$ лежит только в одном из $V_{i}$, скажем $V_{i(r)}$. Индекс $i(r)$ неограниченно растет, когда $r \rightarrow \infty$. Действительно, если это не так, то с учетом неравенства $\delta<1$ нетрудно заметить, что хотя бы одно из $V_{i}$ должно быть тогда неограниченным, что неверно. Следовательно, $i(r)$, а вместе с ним и $\left|\lambda_{k_{i(r)}}\right|$ стремятся к $+\infty$ при $r \rightarrow \infty$. Тогда, как мы отмечали, $V_{i(r)} \subset B\left(w, 88 \delta_{r}|w|\right)$, где $w \in S_{r}$ и $\delta_{r} \rightarrow 0$ при $r \rightarrow \infty$. Это означает, в частности, что $S_{r}$ не может лежать в $V_{i(r)}$ при больших $r$. Полученное противоречие завершает доказательство теоремы.

ЗАмЕчАниЕ 4.7. Утверждение теоремы 4.6 сильнее утверждения (i) теоремы 4.3, сформулированного для ограниченной области. Действительно, согласно [55, лемма 2.7] неравенство $h_{\varphi}(y) \geqslant H_{K_{m+1}}(y)$ влечет за собой сушествование последовательности $\left\{z_{j}\right\}$ такой, что $z_{j} /\left|z_{j}\right| \rightarrow y,\left|z_{j+1}\right| /\left|z_{j}\right| \rightarrow 1,\left|z_{j}\right| \rightarrow \infty$ и $\ln \left|\varphi\left(z_{j}\right)\right| \geqslant H_{K_{m}}\left(z_{j}\right), j \geqslant 1$. Как легко заметить, это означает, что для любого $\delta>0$ множество $R_{m}^{\delta}$ содержит внешность некоторого круга с центром в нуле, а не только окрестности точек $\lambda_{k}$.

СлЕДСТВИЕ 4.8. В условиях теоремь 4.6 для каждого $m \geqslant 1$ существует $j \geqslant 1$ mакой, что $\underline{h}_{\Psi_{j}} \geqslant H_{K_{m}}$.

ДокАЗАТЕЛЬСтвО. Пусть $m \geqslant 1$. По теореме 4.6 найдем функцию $\varphi_{m} \in I_{\Lambda}(D)$ такую, что $\underline{h}_{\varphi_{m}} \geqslant H_{K_{m}}$. Поскольку $\varphi_{m} \in \mathbf{P}_{D}$, то для некоторого $j \geqslant 1$ пространство $P_{j}$ содержит $\varphi_{m}$. Пусть $d=\left\|\varphi_{m}\right\|_{j}$. Тогда по определению $\Psi_{j}$ имеем $\Psi_{j} \geqslant \ln \left|\varphi_{m} / d\right|$. Следовательно,

$$
\underline{h}_{\Psi_{j}}(z) \geqslant \underline{h}_{\varphi_{m} / d}(z)=\underline{h}_{\varphi_{m}}(z) \geqslant H_{K_{m}}(z) \quad \forall z .
$$

Для ограниченной выпуклой области $D$ введем еще функцию

$$
\Psi(z)=\varlimsup_{w \rightarrow z} \sup \left\{\ln |\varphi(w)|: \varphi \in I_{\Lambda}(\mathbb{C}), \sup _{y \in \mathbb{C}}|\varphi(y)| \exp \left(-H_{D}(y)\right) \leqslant 1\right\} .
$$

По тем же соображениям, что и в доказательстве теоремы $4.6, \Psi(z)$ - субгармоническая функция. 
СлЕДСТВИЕ 4.9. В условиях теоремы 4.6 функиия $\Psi(z)$ имеет регулярный рост всюду в плоскости и ее верхний индикатор равен $H_{D}$, m. е. $\underline{h}_{\Psi} \equiv$ $h_{\Psi} \equiv H_{D}$.

ДокАЗАТЕЛЬСТво. Из определения $\Psi$ следует, что $h_{\Psi} \leqslant H_{D}$. Нетрудно заметить также, что $\Psi \geqslant \Psi_{j}$ для любого $j \geqslant 1$. Тогда по следствию $4.8 \underline{h}_{\Psi} \geqslant H_{K_{m}}$, $m \geqslant 1$. Отсюда с учетом неравенства $\underline{h}_{\Psi} \leqslant h_{\Psi}$ и того, что $\left\{K_{m}\right\}$ исчерпывает $D$, получаем требуемое.

Теорема 4.10. Пусть $\underline{h}_{\Psi} \equiv h_{\Psi} \equiv H_{D}$. Тогда существует челая функчия $\varphi \in I_{\Lambda}(\mathbb{C})$, которая имеет регулярный рост всюду в плоскости и ее верхний индикатор равен $H_{D}$, т.е. $\underline{h}_{\varphi} \equiv h_{\varphi} \equiv H_{D}$.

ДОКАЗАТЕЛЬСТВО. Положим

$$
\widetilde{\Psi}(z)=\varlimsup_{w \rightarrow z} \sup \left\{\ln |\varphi(w)|-\ln \left|f_{\Lambda}(w)\right|: \varphi \in I_{\Lambda}(\mathbb{C}), \sup _{y \in \mathbb{C}}|\varphi(y)| \exp \left(-H_{D}(y)\right) \leqslant 1\right\} .
$$

Ранее мы показали, что функция $\widetilde{\Psi}$ субгармонична во всей плоскости и удовлетворяет оценке вида (4.8). Тогда в силу [53, теорема 5] найдутся целая функция $\tilde{\varphi}$, постоянная $C>0$ и исключительное множество $E \subset \mathbb{C}$ такие, что

$$
|\ln | \tilde{\varphi}(z)|-\widetilde{\Psi}(z)| \leqslant C \ln |z|, \quad z \in \mathbb{C} \backslash E,
$$

причем множество $E$ может быть покрыто кружками $B_{i}=B\left(z_{i}, r_{i}\right), i \geqslant 1$, такими, что $\sum r_{i}=A<\infty$. Положим $\varphi(z)=\tilde{\varphi}(z) f_{\Lambda}(z)$. Как и в теореме 4.3 , устанавливается неравенство $h_{\varphi} \leqslant H_{D}$. Если $\underline{h}_{\varphi}(y) \geqslant H_{D}$, то утверждение теоремы будет выполнено. Предположим противное, т. е. $\underline{h}_{\varphi}(y)<H_{D}(y)-3 \tilde{\varepsilon}$ для некоторых $\tilde{\varepsilon}>0$ и $y \in \mathbf{S}$. Тогда, как и в теореме 4.6 , существуют $\delta \in(0,1)$ и последовательность $\left\{t_{r}\right\}, t_{r} \rightarrow+\infty$, такие, что

$$
\ln |\varphi(z)| \leqslant H_{D}(z)-\tilde{\varepsilon}|z|, \quad z \in B\left(t_{r} y, \delta t_{r}\right), \quad r=1,2, \ldots
$$

Пусть $\varepsilon \in(0, \tilde{\varepsilon} / 25)$. Из (4.20) и определений $\Psi(z), \widetilde{\Psi}(z)$ следует оценка

$$
\ln |\varphi(z)| \geqslant \Psi(z)-\varepsilon|z|, \quad z \notin E, \quad|z|>T,
$$

для некоторого $T>0$. По условию $\underline{h}_{\Psi}(y) \geqslant H_{D}(y)$. Тогда в силу [55, лемма 2.7$]$ с учетом неравенства (3.13) сушествует последовательность $\left\{z_{j}\right\}$ такая, что

$$
\begin{array}{cl}
\left|z_{j}\right| \uparrow+\infty, \quad\left|z_{j} /\right| z_{j}|-y|<\delta / 4, & \left|z_{j+1}\right| /\left|z_{j}\right|<1+\delta / 4, \\
\Psi\left(z_{j}\right) \geqslant H_{D}\left(z_{j}\right)-\varepsilon\left|z_{j}\right|, & j=1,2, \ldots
\end{array}
$$

Поскольку $\left|z_{j}\right| \uparrow+\infty$, то для каждого $r$, начиная с некоторого $r_{0}$, найдется номер $j(r)$ такой, что $\left|z_{j(r)}\right| \leqslant t_{r} \leqslant\left|z_{j(r)+1}\right|$. Из свойств $\left\{z_{j}\right\}$ следует, что имеет место вложение $B\left(z_{j(r)}, \delta\left|z_{j(r)}\right| / 2\right) \subset B\left(t_{r} y, \delta t_{r}\right), r \geqslant r_{0}$. Учитьвая еще определение $\Psi(z)$ и неравенство (3.13) для любого $r \geqslant r_{0}$ найдем точку $\tilde{z}_{r}$ и целую функцию $\varphi_{r}$, которые удовлетворяют условиям

$$
\begin{gathered}
B\left(\tilde{z}_{r}, \delta\left|\tilde{z}_{r}\right| / 3\right) \subset B\left(t_{r} y, \delta t_{r}\right), \quad \ln \left|\varphi_{r}(z)\right| \leqslant H_{D}(z) \quad \forall z \\
\ln \left|\varphi_{r}\left(\tilde{z}_{r}\right)\right| \geqslant H_{D}\left(\tilde{z}_{r}\right)-2 \varepsilon\left|\tilde{z}_{r}\right| .
\end{gathered}
$$


Отсюда для функции $\tilde{\varphi}_{r}(z)=\varphi_{r}(y) / \varphi_{r}\left(\tilde{z}_{r}\right)$ с учетом неравенства $(3.13)$ (уменьшая при необходимости $\delta>0)$ получаем соотношения

$$
\tilde{\varphi}_{r}\left(\tilde{z}_{r}\right)=1, \quad \ln \left|\tilde{\varphi}_{r}(z)\right| \leqslant 3 \varepsilon\left|\tilde{z}_{r}\right|, \quad z \in B\left(\tilde{z}_{r}, 2 \delta\left|\tilde{z}_{r}\right|\right)
$$

Тогда по теореме об оценке снизу модуля аналитической функции (см., например, $[48$, гл. $1, \S 4$, теорема 4.2]) имеет место неравенство

$$
\ln \left|\tilde{\varphi}_{r}(z)\right| \geqslant-(3+\ln 24)\left(3 \varepsilon\left|\tilde{z}_{r}\right|\right) \geqslant-21 \varepsilon\left|\tilde{z}_{r}\right|
$$

которое выполнено в $B\left(\tilde{z}_{r}, \delta\left|\tilde{z}_{r}\right| / 3\right)$, но вне исключительных кружков $\widetilde{B}_{r, s}$ с суммой радиусов, равной $\delta\left|\tilde{z}_{r}\right| / 12$. Увеличивая $r_{0}$, можно считать, что $2 A<\delta\left|\tilde{z}_{r}\right| / 6$ и $(1-\delta / 3)\left|\tilde{z}_{r}\right|>T$ при $r \geqslant r_{0}$. Тогда сумма диаметров всех кружков $\widetilde{B}_{r, s}$ и $B_{i}$ строго меньше $\delta\left|\tilde{z}_{r}\right| / 3$ при $r \geqslant r_{0}$. Следовательно, в $B\left(\tilde{z}_{r}, \delta\left|\tilde{z}_{r}\right| / 3\right), r \geqslant r_{0}$, найдется точка $w_{r}$ (и даже целая окружность), для которой выполнены неравенства (4.22) и (4.23). В силу (4.23) и оценки на $\ln \left|\varphi_{r}\left(\tilde{z}_{r}\right)\right|$ с учетом (3.13) имеем

$$
\ln \left|\varphi_{r}\left(w_{r}\right)\right| \geqslant H_{D}\left(w_{r}\right)-24 \varepsilon\left|w_{r}\right|
$$

Отсюда, из (4.22) и выбора $\varepsilon$ при $r \geqslant r_{0}$ следует оценка

$$
\ln \left|\varphi\left(w_{r}\right)\right| \geqslant \ln \left|\varphi_{r}\left(w_{r}\right)\right|-\varepsilon\left|w_{r}\right| \geqslant H_{D}\left(w_{r}\right)-25 \varepsilon\left|w_{r}\right|>H_{D}\left(w_{r}\right)-\tilde{\varepsilon}\left|w_{r}\right|,
$$

которая противоречит (4.21). Теорема доказана.

В заключение параграфа покажем, что дополнительные условия $m_{D}(\Lambda)=0$ и $m(\Lambda)=0$ из теорем 4.2 и 4.6 на самом деле в некоторых случаях являются следствием разрешимости интерполяционной задачи и фундаментального принципа. Перед этим введем еще одну характеристику $\Lambda$ :

$$
G_{\Lambda}=\varlimsup_{k \rightarrow \infty}\left|\lambda_{k}\right|^{-1} \min _{j \neq k}\left|\lambda_{k}-\lambda_{j}\right|
$$

Кроме того, для $D \neq \mathbb{C}$ положим также

$$
G_{\Lambda}(F)=\inf _{E \supset F} G_{\Lambda(E)}, \quad G_{\Lambda}(D)=\sup _{F \subset \mathbf{S} \backslash J(D)} G_{\Lambda}(F)
$$

где $E$ - произвольное открытое на $\mathbf{S}$ множество, содержащее $F$, а $F$ - компактное подмножество $\mathbf{S} \backslash J(D)$. В отличие от величины $S_{\Lambda}$, которая дает оценку снизу на расстояния от точки $\lambda_{k}$ до других точек $\lambda_{j}$, величина $G_{\Lambda}$ обеспечивает подобную оценку сверху.

ТЕОРема 4.11. Пусть $D-$ выпуклая область и $\Lambda-$ такая, что $G_{\Lambda}(D)=0$. Предположим, что $\Phi \subset \Sigma\left(\mathbf{P}_{D} / I_{\Lambda}(D)\right)$. Тогда $m_{D}(\Lambda)=0$. 
ДоКАЗАТЕЛЬСТво. Пусть $F$ - произвольньй компакт из $\mathbf{S} \backslash J(D)$. Предположим, что $m_{F}(\Lambda)>\tilde{\varepsilon}>0$. Тогда найдутся подпоследовательность $\left\{\lambda_{k_{j}}\right\}$ и точка $x_{0} \in F$ такие, что $\lambda_{k_{j}} /\left|\lambda_{k_{j}}\right|$ сходится к $x_{0},\left|\lambda_{k_{j+1}}\right| \geqslant 2\left|\lambda_{k_{j}}\right|$ и $m_{k_{j}} \geqslant \tilde{\varepsilon}\left|\lambda_{k_{j}}\right|, j \geqslant 1$. Для каждого $j \geqslant 1$ выберем номер $\tilde{k}_{j}$, удовлетворяющий условию

$$
\left|\lambda_{k_{j}}-\lambda_{\tilde{k}_{j}}\right|=\min _{k \neq k_{j}}\left|\lambda_{k_{j}}-\lambda_{k}\right|
$$

По условию и определению величины $G_{\Lambda}(D)$ имеем $\left|\lambda_{k_{j}}\right|^{-1}\left|\lambda_{k_{j}}-\lambda_{\tilde{k}_{j}}\right| \rightarrow 0$ при $j \rightarrow \infty$. Отсюда следует, что $\lambda_{\tilde{k}_{j}} /\left|\lambda_{\tilde{k}_{j}}\right|$ также сходится к $x_{0}$ и, кроме того, $\lambda_{\tilde{k}_{j}} \in$ $B\left(\lambda_{k_{j}}, \delta_{j}\left|\lambda_{k_{j}}\right|\right)$, где $\delta_{j} \rightarrow 0$ при $j \rightarrow \infty$. Поскольку $\left|\lambda_{k_{j+1}}\right| \geqslant 2\left|\lambda_{k_{j}}\right|$, то в круге $B\left(\lambda_{k_{j}}, 2^{-1}\left|\lambda_{k_{j}}\right|\right)$ нет точек $\lambda_{k_{p}}$ при $p \neq j$. Поэтому можно считать, что для каждого $j \geqslant 1$ точка $\lambda_{\tilde{k}_{j}}$ не совпадает ни с одной из точек $\lambda_{k_{p}}, p=1,2, \ldots$ Пусть $\varepsilon \in(0, \tilde{\varepsilon} / 4)$. Выберем номер $m$ так, что $H_{K_{m}}\left(x_{0}\right) \geqslant H_{D}\left(x_{0}\right)-\varepsilon$. Рассмотрим последовательность $b=\left\{b_{k, n}\right\}_{k=1, n=1}^{\infty, m_{k}}$, где $b_{\tilde{k}_{j}, 1}=\exp \left(H_{K_{m}}\left(\lambda_{\tilde{k}_{j}}\right)\right), j \geqslant 1$, и $b_{k, n}=0$ для всех остальных $k, n$. Тогда $b \in \Phi$. Поэтому согласно условию существует $f \in \mathbf{P}_{D}$ такая, что $\widetilde{\Sigma}(f)=b$, т.е. $f^{(n-1)}\left(\lambda_{k}\right)=b_{k, n}, k \geqslant 1, n=\overline{1, m_{k}}$. В силу непрерывности $h_{f}$, выбора $m$ и неравенства $h_{f}(z)<H_{D}(z), z \neq 0$, для некоторого $\delta \in(0,1 / 2)$ имеем

$$
h_{f}(z) \leqslant H_{D}\left(x_{0}\right) \leqslant H_{K_{m}}\left(x_{0}\right)+\varepsilon, \quad z \in B\left(x_{0}, 2 \delta\right) .
$$

Тогда по лемме 3.2 для некоторого $T>0$ верна оценка

$$
\begin{gathered}
M_{\tilde{\Lambda}}(y, \delta / 4) \leqslant H_{K_{m}}\left(x_{0}\right)|y|-\ln |\varphi(y)|+2 \varepsilon|y|, \\
y /|y| \in B\left(x_{0}, \delta\right), \quad|y|>T, \quad f(y) \neq 0,
\end{gathered}
$$

где $\tilde{\Lambda}=\left\{\tilde{\lambda}_{s}, \widetilde{m}_{s}\right\}$ - последовательность нулей и их кратностей функции $f$. Отметим, что по построению $f$ обрашается в нуль в точках $\lambda_{k_{j}}$ с кратностью, не меньшей, чем $m_{k_{j}}$. Поскольку $\lambda_{\tilde{k}_{j}} /\left|\lambda_{\tilde{k}_{j}}\right|$ сходится к $x_{0}$ и $\lambda_{\tilde{k}_{j}} \in B\left(\lambda_{k_{j}}, \delta_{j}\left|\lambda_{k_{j}}\right|\right)$, то по предыдущему

$$
\begin{aligned}
m_{k_{j}} & \leqslant M_{\tilde{\Lambda}}\left(\lambda_{\tilde{k}_{j}}, \delta / 4\right) \leqslant H_{K_{m}}\left(x_{0}\right)\left|\lambda_{\tilde{k}_{j}}\right|-\ln \left|\varphi\left(\lambda_{\tilde{k}_{j}}\right)\right|+2 \varepsilon\left|\lambda_{\tilde{k}_{j}}\right| \\
& =H_{K_{m}}\left(x_{0}\right)\left|\lambda_{\tilde{k}_{j}}\right|-H_{K_{m}}\left(\lambda_{\tilde{k}_{j}}\right)+2 \varepsilon\left|\lambda_{\tilde{k}_{j}}\right|, \quad j \geqslant j_{0} .
\end{aligned}
$$

Отсюда, учитывая еще непрерывность $H_{K_{m}}$ и выбор $\varepsilon$, получаем

$$
m_{k_{j}} \leqslant 3 \varepsilon\left|\lambda_{\tilde{k}_{j}}\right| \leqslant 3 \varepsilon\left(1+\delta_{j}\right)\left|\lambda_{k_{j}}\right| \leqslant 4 \varepsilon\left|\lambda_{k_{j}}\right|<\tilde{\varepsilon}\left|\lambda_{k_{j}}\right|, \quad j \geqslant j_{1} .
$$

Это противоречит выбору $\left\{\lambda_{k_{j}}\right\}$. Теорема доказана.

Пусть $D$ - ограниченная выпуклая область и $w \in \mathbf{S}$. Через $L_{D}(w)$ и $\Pi_{D}(w)$ обозначим соответственно опорную прямую и опорную полуплоскость к $D$ в направлении $w$, т. е. $L_{D}(w)=\left\{z: \operatorname{Re}(z w)=H_{D}(w)\right\}, \Pi_{D}(w)=\left\{z: \operatorname{Re}(z w)<H_{D}(w)\right\}$. Для каждого $w \in \mathbf{S}$ область $D$ лежит в $\Pi_{D}(w)$, а прямая $L_{D}(w)$ не пересекает $D$ и содержит хотя бы одну точку $z \in \partial D$. Множество всех таких $z$ обозначим $\Gamma_{D}(w)$. 
Через каждую точку $z \in \partial D$ проходит хотя бы одна опорная прямая. Если область $D$ гладкая, то такая прямая лишь одна.

Будем говорить, что $w \in \mathbf{S}$ - круговое направление по отношению к $D$, если для некоторого $z \in \Gamma_{D}(w)$ выполнено одно из следуюших условий:

1) $z=0$;

2) $B(0,|z|) \cap D=\varnothing$;

3) для каждого $\delta \in(0,|z|)$ дуга $\partial B(0,|z|) \cap B(0, \delta)$ окружности $\partial B(0,|z|)$ содержит точки множества $\bar{D}$ по обе стороны от $z$.

Нетрудно заметить, что если $w \in \mathbf{S}$ - круговое направление по отношению к $D$, то для некоторого $z \in \Gamma_{D}(w)$ либо $z=0$, либо прямая $L_{D}(w)$ перпендикулярна отрезку $[0, z]$. Существуют по крайней мере две точки $z \in \partial D$, обладающие указанными свойствами. Это - самая близкая и самая дальняя относительно начала координат точки гранищы. В силу выпуклости $D$ имеется лишь одна граничная точка $z$, ближайшая к началу координат. При этом направление $w$, для которого $z \in \Gamma_{D}(w)$, всегда является круговым по отношению к $D$. В отличие от этого точек $z \in \partial D$, наиболее удаленных от начала координат, может быть несколько и даже бесконечно много, но при этом, в общем случае, направление $w$, для которого $z \in \Gamma_{D}(w)$, не обязано быть круговым по отношению к $D$. Например, $\operatorname{ecли~} D-$ круг с центром в нуле, то все $w \in \mathbf{S}$ являются круговыми направлениями по отношению к $D$. Если $D-$ круг, центр которого отличен от начала координат, то сушествует лишь одно направление, круговое по отношению к $D$.

ТЕОРЕМА 4.12. Пусть $D$ - выпуклая гладкая ограниченная область, $F \subset \mathbf{S}$, $W$ - нетривиальное замкнутое инвариантное подпространство в $H(D)$ со спектром $\Lambda=\left\{\lambda_{k}, m_{k}\right\}$. Предположим, что выполнено следующее:

1) каждое $w \in F \cap N(\Lambda)$ не является круговым направлением по отношению $\kappa D$;

2) в $W$ имеет место фундаментальный принщип, т.е. любая функиия $\varphi \in W$ представляется рядом (2.11), сходящимся в топологии $H(D)$.

Тогда $m_{F}(\Lambda)=0$.

ДокАЗАТЕЛЬСтво. Предположим, что $m_{F}(\Lambda)>\tilde{\varepsilon}>0$. Тогда найдутся подпоследовательность $\left\{\lambda_{k(j)}\right\}$ и точка $w_{0} \in F$ такие, что $\lambda_{k(j)} /\left|\lambda_{k(j)}\right|$ сходится к $w_{0}$, $\left|\lambda_{k(j)}\right| \rightarrow \infty$ и $m_{k(j)} \geqslant \tilde{\varepsilon}\left|\lambda_{k(j)}\right|, j \geqslant 1$. Пусть $\widetilde{W}$ - замыкание линейной оболочки системы $\left\{z^{n-1} \exp \left(\lambda_{k(j)} z\right), n=1, \ldots, m_{k(j)}, j \geqslant 1\right\}$ в топологии $H(D)$. Тогда $\widetilde{W}$ - нетривиальное замкнутое инвариантное подпространство в $H(D)$, допускающее спектральный синтез, причем $\widetilde{W} \subset W$. Ближайшую к началу координат точку множества $\Gamma_{D}\left(w_{0}\right)$ обозначим $z_{0}$. По условию 1$)$ теоремы $z_{0} \neq 0$ и, так как $D \subset \Pi_{D}\left(w_{0}\right)$, пересечение $B\left(0,\left|z_{0}\right|\right) \cap \Pi_{D}\left(w_{0}\right)$ не пусто. Покажем, что все функции из $\widetilde{W}$ аналитически продолжаются в область $B\left(0,\left|z_{0}\right|\right) \cap \Pi_{D}\left(w_{0}\right)$.

Пусть $\varphi \in \widetilde{W}$. По условию 2) с учетом леммы 2.6 функция $\varphi$ разлагается в ряд (2.11), где $d_{k, n}=\nu_{k, n}(\varphi)$, а $\left\{\nu_{k, n}\right\}$ - последовательность функционалов из $H^{*}(D)$, биортогональная к системе (1.4). Если $k$ не совпадает ни с одним из $k(j)$ и $n=1, \ldots, m_{k}$, то $\nu_{k, n}\left(z^{p-1} \exp \left(\lambda_{k(j)} z\right)\right)=0$ для всех $p=1, \ldots, m_{k(j)}, j \geqslant 1$. 
Отсюда следует, что $d_{k, n}=\nu_{k, n}(\varphi)=0, n=1, \ldots, m_{k}, k \neq k(j)$, т.е.

$$
\varphi(z)=\sum_{j=1, n=0}^{\infty, m_{k(j)}-1} d_{k(j), n} z^{n} \exp \left(\lambda_{k(j)} z\right),
$$

причем ряд сходится в $H(D)$. Пусть $K$ - произвольный компакт из области $B\left(0,\left|z_{0}\right|\right) \cap \Pi_{D}\left(w_{0}\right)$. Поскольку $z_{0} \in \Gamma_{D}\left(w_{0}\right)$, то сушествуют число $\tau>0$ и точка $z_{1} \in D$ такие, что $\left|z_{1}\right| \geqslant|z|$ и $\operatorname{Re}\left(z_{1} w_{0}\right)>\operatorname{Re}\left(z w_{0}\right)+\tau$ для всех $z \in K$. Из последнего неравенства и сходимости $\lambda_{k(j)} /\left|\lambda_{k(j)}\right|$ к $w_{0}$ следует, что для некоторого $a>0$ верна оценка

$$
a+\operatorname{Re}\left(z_{1} \lambda_{k(j)}\right)>\operatorname{Re}\left(z \lambda_{k(j)}\right)+\tau\left|\lambda_{k(j)}\right|, \quad z \in K, \quad j \geqslant 1 .
$$

Тогда в силу ограниченности членов ряда (4.24) в точке $z_{1}$ по тем же соображениям, что и в предложении 2.7, получаем

$$
\begin{aligned}
& \sum_{j=1, n=0}^{\infty, m_{k(j)}-1}\left|d_{k(j), n}\right| \sup _{z \in K}\left|z^{n} \exp \left(\lambda_{k(j)} z\right)\right| \\
& \leqslant C \sum_{j=1, n=0}^{\infty, m_{k(j)}-1} \sup _{z \in K}\left(\left|z / z_{1}\right|^{n} \exp \left(\operatorname{Re}\left(\lambda_{k(j)}\left(z-z_{1}\right)\right)\right)\right) \\
& \leqslant C_{1} \sum_{j=1, n=0}^{\infty, m_{k(j)}-1} \exp \left(-\tau\left|\lambda_{k(j)}\right|\right)<\infty .
\end{aligned}
$$

Таким образом, каждая функция $\varphi \in \widetilde{W}$ аналитична в области $D \cup\left(B\left(0,\left|z_{0}\right|\right) \cap\right.$ $\left.\Pi_{D}\left(w_{0}\right)\right)$ и представляется в этой области рядом (4.24), который сходится равномерно на компактах. Покажем теперь, что в этой области содержится некоторая специальная выпуклая область $\widetilde{D}$, которая, в свою очеред, содержит $D$.

Из условия 1$)$ следует, что $D$ пересекает круг $B\left(0,\left|z_{0}\right|\right)$ и последний не лежит в $D$. Если $D \subset B\left(0,\left|z_{0}\right|\right)$, то в качестве $\widetilde{D}$ возьмем $B\left(0,\left|z_{0}\right|\right) \cap \Pi_{D}\left(w_{0}\right)$. В противном случае окружность $\partial B\left(0,\left|z_{0}\right|\right)$ содержит граничные точки $D$, отличные от $z_{0}$, и по условию 1$)$ найдем $z_{2} \in \partial D \cap \partial B\left(0,\left|z_{0}\right|\right)$ такую, что открытая дуга $\gamma$ окружности $\partial B\left(0,\left|z_{0}\right|\right)$ с концами $z_{0}$ и $z_{2}$ не пересекает $\bar{D}$.

Как отмечалось вьше, найдется $w_{2} \in \mathbf{S}$, для которого множество $\Gamma_{d}\left(w_{2}\right)$ содержит $z_{2}$. Поскольку $z_{0}-$ ближайшая к началу координат точка множества $\Gamma_{D}\left(w_{0}\right)$, то $w_{2} \neq w_{0}$. Тогда с учетом гладкости $D$ прямая $L_{D}\left(w_{2}\right)$ не содержит $z_{0}$. Предположим вначале, что $B\left(0,\left|z_{0}\right|\right) \subset \Pi_{D}\left(w_{0}\right)$. Определим точку $z_{3} \in \partial B\left(0,\left|z_{0}\right|\right)$. Если прямая $L_{D}\left(w_{2}\right)$ не пересекает $\gamma$, то положим $z_{3}=z_{2}$. В противном случае в качестве $z_{3}$ возьмем точку пересечения $L_{D}\left(w_{2}\right)$ и $\gamma$. Пусть $\gamma_{1}$ - дуга с конщами $z_{0}$ и $z_{3}$, лежащая на $\gamma$. Тогда $\widetilde{D}$ определим как внутренность выпуклой оболочки множества $\bar{D} \cup \bar{\gamma}_{1}$. Пусть теперь $B\left(0,\left|z_{0}\right|\right)$ не лежит целиком в $\Pi_{D}\left(w_{0}\right)$ и $\gamma_{2}$ - хорда окружности $\partial B\left(0,\left|z_{0}\right|\right)$, лежашая на прямой $L_{D}\left(w_{0}\right)$. Если $L_{D}\left(w_{2}\right)$ не пересекает $\gamma_{2}$, то положим $\gamma_{3}=\gamma_{2}$. В противном случае в качестве $\gamma_{3}$ возьмем отрезок $\left[z_{0}, z_{4}\right]$, где $z_{4}$ - точка пересечения $L_{D}\left(w_{2}\right)$ и $\gamma_{2}$, которая не совпадает с $z_{0}$ (если 
$z_{0}=z_{4}$, то $z_{0} \in L_{D}\left(w_{2}\right)$, что невозможно в силу гладкости области $\left.D\right)$. Определим $\widetilde{D}$ как внутренность выпуклой оболочки объединения $\bar{D} \cup \gamma_{3}$. Очевидно, что $D \subset \widetilde{D}$. Отметим, что $\widetilde{D}$ можно представить в следующем виде (см., например, [2]):

$$
\widetilde{D}=\operatorname{int}\left(\bigcup_{z \in \bar{D}, y \in \gamma^{\prime}}\{t z+(1-t) y, t \in[0,1]\}\right)=\operatorname{int}\left(\bigcup_{z \in \bar{D}, y \in \gamma^{\prime}}[z, y]\right)
$$

где $\gamma^{\prime}-$ дуга $\bar{\gamma}_{1}$ или отрезок $\gamma_{3}$. По построению кривая $\gamma^{\prime}$ лежит в угле $\Delta$, которьй является пересечением полуплоскостей $\overline{\Pi_{D}\left(w_{0}\right)}$ и $\overline{\Pi_{D}\left(w_{2}\right)}$. Там же согласно определению опорной функции лежит и $\bar{D}$. Следовательно, $\widetilde{D} \subset \Delta$. Остается показать, что имеет место вложение $\widetilde{D} \subset B\left(0,\left|z_{0}\right|\right) \cup D$.

Отрезок $\left[z_{0}, z_{2}\right]$ делит угол $\Delta$ на две части $\Delta_{1}$ и $\Delta_{2}$, причем $\gamma^{\prime}$ целиком лежит в одной из них, скажем $\Delta_{1}$. Кроме того, $\gamma^{\prime} \subset \overline{B\left(0,\left|z_{0}\right|\right)}$. Пусть $z \in \bar{D}$ и $y \in \gamma^{\prime}$. Если $z \in \overline{B\left(0,\left|z_{0}\right|\right)}$, то и $[z, y] \subset \overline{B\left(0,\left|z_{0}\right|\right)}$. Предположим, что $z$ не принадлежит кругу $\overline{B\left(0,\left|z_{0}\right|\right)}$. Тогда $z \in \Delta_{2}$. (Действительно, в противном случае отрезок $\left[z_{0}, z\right]$, лежащий в $\bar{D}$, пересекает дугу $\gamma$, а это противоречит выбору $\gamma$.) Поскольку $y \in \Delta_{1}$ и $\left[z_{0}, z_{2}\right] \subset \overline{B\left(0,\left|z_{0}\right|\right)}$, то отрезки $\left[z_{0}, z_{2}\right]$ и $[z, y]$ пересекаются в некоторой точке $x \in \overline{B\left(0,\left|z_{0}\right|\right)}$. Тогда $[x, y] \subset \overline{B\left(0,\left|z_{0}\right|\right)}$, а в силу выпуклости множества $\bar{D}$ оно содержит $[z, x]$. Таким образом, мы показали, что требуемое вложение имеет место. По доказанному это означает, что каждая функция $\varphi \in \widetilde{W}$ аналитически продолжается в выпуклую область $\widetilde{D} \supset D$ и представляется там рядом (4.24), который сходится равномерно на компактах.

Положим $J(D, \widetilde{D})=\left\{w \in \mathbf{S}: H_{D}(w)<H_{\widetilde{D}}(w)\right\}$. Множество $J(D, \widetilde{D})$ не содержит точку $w_{0}\left(H_{\widetilde{D}}\left(w_{0}\right) \leqslant H_{D}\left(w_{0}\right)\right.$, так как $\left.\widetilde{D} \subset \Pi_{D}\left(w_{0}\right)\right)$, которая является единственной предельной точкой последовательности $\left\{\lambda_{k(j)} /\left|\lambda_{k(j)}\right|\right\}$. Тогда из $[56$, теорема 5] следует, что для каждого $w \in \partial J(D, \widetilde{D}) \cap \mathbf{S}$ и любых $\delta, \varepsilon>0$ сушествуют $\xi \in B(w, \delta) \cap \mathbf{S}$ и $f \in \mathbf{P}_{D}$, обрашаюшаяся в нуль в точках $\lambda_{k(j)}$ с кратностью, не меньшей, чем $m_{k(j)}$, удовлетворяюшие условию

$$
\underline{h}_{f}(\xi) \geqslant H_{D}(\xi)-H_{D_{1}}(\xi)-\varepsilon,
$$

где $D_{1}=\left\{z: \operatorname{Re}(z \zeta)<H_{D}(\zeta)-h_{f}(\zeta), \zeta \in \mathbf{S}\right\}$.

В силу [53, теорема 5] найдутся целая функция $f_{1}(z)$, постоянная $C>0$ и исключительное множество $E \subset \mathbb{C}$ такие, что

$$
|\ln | f_{1}(z)\left|-H_{D_{1}}(z)\right| \leqslant C \ln |z|, \quad z \in \mathbb{C} \backslash E
$$

причем $E$ может быть покрыто кружками $B_{i}=B\left(z_{i}, r_{i}\right), i \geqslant 1$, такими, что $\sum r_{i}=A<\infty$. Положим $\tilde{f}(z)=f(z) f_{1}(z)$. Из определения $D_{1}$ следует, что $h_{f}(\zeta) \leqslant H_{D}(\zeta)-H_{D_{1}}(\zeta) \forall \zeta$. Поэтому, используя оценку (4.25), предложение 9.3 из [54], лемму 2.7 из [55], теорему об оценке снизу модуля аналитической функции и рассуждения, схожие с теми, которые мы проводили в теоремах 4.3, 4.6, 4.10, нетрудно установить, что

$$
\underline{h}_{\tilde{f}}(\xi) \geqslant H_{D}(\xi)-b \varepsilon
$$


где $b>0$ не зависит от $w, \delta, \varepsilon$. При этом имеет место также неравенство

$$
h_{\tilde{f}}(\zeta) \leqslant h_{f}(\zeta)+H_{D_{1}}(\zeta) \leqslant H_{D}(\zeta) \quad \forall \zeta .
$$

Покажем, что $w_{0} \in \partial J(D, \widetilde{D})$. По выбору дуга $\gamma_{1}$ не пересекает $\bar{D}$. Отрезок $\gamma_{3}$, лежащий на прямой $L_{D}\left(w_{0}\right)$, пересекает $\bar{D}$ лишь в точке $z_{0}$, так как в противном случае $z_{0}$ не будет ближайшей к началу координат точкой множества $\Gamma_{D}\left(w_{0}\right)$. Пусть последовательность $\left\{y_{p}\right\}, y_{p} \in \gamma^{\prime}, y_{p} \neq z_{0}$, сходится к $z_{0}$. Поскольку $y_{p} \notin \bar{D}$, то найдется $\xi_{p} \in \mathbf{S}$ такая, что $H_{\widetilde{D}}\left(\xi_{p}\right) \geqslant \operatorname{Re}\left(y_{p} \xi_{p}\right)>H_{\widetilde{D}}\left(\xi_{p}\right), p \geqslant 1$. Следовательно, $\xi_{p} \in J(D, \widetilde{D}), p \geqslant 1$. Переходя к подпоследовательности, можно считать, что $\left\{\xi_{p}\right\}$ сходится к $\xi_{0}$. Тогда

$$
H_{D}\left(\xi_{0}\right) \geqslant \operatorname{Re}\left(z_{0} \xi_{0}\right)=\lim _{p \rightarrow \infty} \operatorname{Re}\left(y_{p} \xi_{p}\right) \geqslant \lim _{p \rightarrow \infty} H_{D}\left(\xi_{p}\right)=H_{D}\left(\xi_{0}\right),
$$

т. е. прямая $L_{D}\left(\xi_{0}\right)$ проходит через точку $z_{0}$. В силу гладкости $D$ это возможно лишь в случае, когда $\xi_{0}=w_{0}$. Таким образом, в (4.26) точку $\xi$ можно выбрать сколь угодно близкой к $w_{0}$. Поэтому, учитывая еще (4.27) и подбирая подходящие $\varepsilon>0$ и $\delta>0$ (так, чтобы для $h_{0}(z)=H_{D}(z)$ при $\tau=5 \delta$ было выполнено $(3.13)$ ), по лемме 3.2 , как и вьше, получаем противоречие с неравенством $m_{F}(\Lambda)>\tilde{\varepsilon}$. Теорема доказана.

\section{§5. Критерии интерполяции и фундаментального принципа}

Прежде всего сфформулируем самый общий критерий, пригодный для произвольной (ограниченной или неограниченной) выпуклой области.

ТЕОРема 5.1. Пусть $D$ - выпуклая область, $W$ - нетривиальное замкнутое инвариантное подпространство в $H(D)$ со спектром $\Lambda=\left\{\lambda_{k}, m_{k}\right\}$, допускающее спектральный синтез. Предположим, что $m_{D}(\Lambda)=0$. Тогда следующие утвержсденя әквивалентны:

1) оператор $\Sigma: \mathbf{P}_{D} / I_{\Lambda}(D) \rightarrow \Phi$ является изоморфизмом линейных топологических пространств;

2) каждая функиия $\varphi \in W$ представляется рядом (2.11), который сходится в каждой точке области $D$;

3) система (1.4) является базисом Кете в $W$;

4) $S_{\Lambda}>-\infty, S_{\Lambda}(D)=0$ и для каждого $m \geqslant 1$ и каждого компакта $F \subset \mathbf{S} \backslash J(D)$ существует $j \geqslant 1$ такое, что для любого $\delta>0$ найдутся $\beta, T>0$, удовлетворяющие условию: $\lambda_{k} \in R_{m, j}^{\delta}$, если $\lambda_{k} /\left|\lambda_{k}\right| \in E(\beta)$ u $\left|\lambda_{k}\right|>T$, где $R_{m, j}=\left\{z: \Psi_{j}(z) \geqslant H_{K_{m}}(z)\right\}, \quad E(\beta)$ - $\beta$-окрестность $F u \Psi_{j}$ определены перед теоремой 4.2 ;

5) $S_{\Lambda}>-\infty, S_{\Lambda}(D)=0$ u для каждого $p \geqslant 1$ и каждого компакта $F \subset \mathbf{S} \backslash J(D)$ cуществует $\varphi \in I_{\Lambda}(D)$ такая, что для любого $\delta>0$ найдутся $\beta, T>0$, удовлетворяющие условию: $\lambda_{k} \in R_{p}^{\delta}$, если $\lambda_{k} /\left|\lambda_{k}\right| \in E(\beta) u\left|\lambda_{k}\right|>T$, где $R_{p}=\left\{z: \ln |\varphi(z)| \geqslant H_{K_{p}}(z)\right\}$.

ДокаЗАТЕльство. Эквивалентность утверждений 1) и 2) доказана в предложении 2.13. Эквивалентность утверждений 2) и 3) вытекает из следствия 2.9. Наконец, эквивалентность утверждений 1), 4) и 5) следует из теорем 3.7, 4.1-4.3 и следствия 4.5 . 
ЗАмЕчАниЕ 5.2. При определении $q_{\Lambda}^{j}(z, y, \delta)$ мы использовали коэффициент 3 в знаменателе. Выбор именно такого коэффициента обусловлен лишш некоторым удобством при рассуждениях. Его вполне можно заменить на любое другое, отличное от нуля число. Действительно, пусть $b \neq 0$ и величины $S_{\Lambda}(b), S_{\Lambda}(D, b)$ определены точно так же, как $S_{\Lambda}, S_{\Lambda}(D)$, по функциям $q_{\Lambda}^{j}(z, y, \delta)$, где число 3 заменено на $b$. Тогда, как нетрудно заметить, имеют место соотношения

$$
\begin{aligned}
S_{\Lambda}(b) \leqslant M_{\Lambda} \ln (3 /|b|)+S_{\Lambda}, & S_{\Lambda} \leqslant M_{\Lambda} \ln (|b| / 3)+S_{\Lambda}(b), \\
S_{\Lambda}(D, b) \leqslant M_{\Lambda}(D) \ln (3 /|b|)+S_{\Lambda}(D), & S_{\Lambda}(D) \leqslant M_{\Lambda}(D) \ln (|b| / 3)+S_{\Lambda}(D, b) .
\end{aligned}
$$

Поскольку в настоящей работе $M_{\Lambda}<+\infty$, то неравенства $S_{\Lambda}>-\infty$ и $S_{\Lambda}(b)>-\infty$ вьполнены или не выполнены одновременно. Если, дополнительно, $M_{\Lambda}(D)=0$, то также одновременно выполнены или не выполнены равенства $S_{\Lambda}(D)=0$ и $S_{\Lambda}(D, b)=0$. Условия 4$\left.), 5\right)$ теоремы 3.7 являются частью необходимых и достаточных условий интерполящии (при ограничении $m_{D}(\Lambda)=0$ ). Поэтому с учетом замечания вслед за доказательством теоремы 3.7 утверждения теоремы 5.1 останутся верными, если соотношения $S_{\Lambda}>-\infty$ и $S_{\Lambda}(D)=0$ заменить в ней на следуюшие: $S_{\Lambda}(b)>-\infty, S_{\Lambda}(D, b)=0$ (для некоторого $b \neq 0$ ).

ЗАмечаниЕ 5.3. Рассмотрим еще так называемый индекс конденсации $\Lambda=$ $\left\{\lambda_{k}, m_{k}\right\}$ :

$$
Q_{\Lambda}=\lim _{\delta \rightarrow 0} \varlimsup_{k \rightarrow \infty}\left|\lambda_{k}\right|^{-1} \int_{0}^{\delta\left|\lambda_{k}\right|} \frac{M_{\Lambda}\left(\lambda_{k}, t /\left|\lambda_{k}\right|\right)-m_{k}}{t} d t .
$$

Положим еще

$$
Q_{\Lambda}(F)=\inf _{E \supset F} Q_{\Lambda(E)}, \quad Q_{\Lambda}(D)=\sup _{F \subset(\mathbf{S} \backslash J(D))} Q_{\Lambda}(F),
$$

где $E$ - произвольное открытое на $\mathbf{S}$ множество, содержащее $F$, а $F$ - компактное подмножество $\mathbf{S} \backslash J(D)$. По формуле Иенсена имеем

$$
\begin{aligned}
\frac{1}{2 \pi} \int_{0}^{2 \pi} & \ln \left|q_{\Lambda}^{k}\left(\lambda_{k}+r \exp (i \theta), \lambda_{k}, \delta\right)\right| d \theta \\
& =\int_{0}^{r} \frac{M_{\Lambda}\left(\lambda_{k}, g(t)\right)-m_{k}}{t} d t+\ln \left|q_{\Lambda}^{k}\left(\lambda_{k}, \lambda_{k}, \delta\right)\right|,
\end{aligned}
$$

где $g(t)=\delta, t \geqslant \delta\left|\lambda_{k}\right|$, и $g(t)=t /\left|\lambda_{k}\right|, t \in\left[0, \delta\left|\lambda_{k}\right|\right]$. Отсюда, используя неравенства $\ln \left|q_{\Lambda}^{k}\left(z, \lambda_{k}, \delta\right)\right| \leqslant 1, z \in B\left(\lambda_{k}, \delta\left|\lambda_{k}\right|\right)$, и $\ln \left|q_{\Lambda}^{k}\left(z, \lambda_{k}, \delta\right)\right| \geqslant 1, z \notin$ $B\left(\lambda_{k}, 5 \delta\left|\lambda_{k}\right|\right)$, получаем

$$
\begin{gathered}
\int_{0}^{\delta\left|\lambda_{k}\right|} \frac{M_{\Lambda}\left(\lambda_{k}, t /\left|\lambda_{k}\right|\right)-m_{k}}{t} d t+\ln \left|q_{\Lambda}^{k}\left(\lambda_{k}, \lambda_{k}, \delta\right)\right| \leqslant 0, \\
M_{\Lambda}\left(\lambda_{k}, \delta\right) \ln 5+\int_{0}^{\delta\left|\lambda_{k}\right|} \frac{M_{\Lambda}\left(\lambda_{k}, t /\left|\lambda_{k}\right|\right)-m_{k}}{t} d t+\ln \left|q_{\Lambda}^{k}\left(\lambda_{k}, \lambda_{k}, \delta\right)\right| \geqslant 0 .
\end{gathered}
$$

Тогда, как и вьше, неравенства $S_{\Lambda}>-\infty, Q_{\Lambda}<+\infty$ и равенства $S_{\Lambda}(D)=0$, $Q_{\Lambda}(D)=0$ (если $M_{\Lambda}(D)=0$ ) выполнены или не выполнены одновременно.

Приведем теперь результат для ограниченной области. Функции $\Psi_{j}$ и $\Psi$ определены ранее по компактам $K_{j}$ и области $D$. 
ТЕОРемА 5.4. Пусть $D$ - ограниченная выпуклая область, $W$ - нетривиальное замкнутое инвариантное подпространство в $H(D)$ со спектром $\Lambda=$ $\left\{\lambda_{k}, m_{k}\right\}$, допускающее спектральный синтез. Предположим, что $m(\Lambda)=0$. Тогда следующие утверждения әквивалентны:

1) оператор $\Sigma: \mathbf{P}_{D} / I_{\Lambda}(D) \rightarrow \Phi$ является изоморфизмом линейных топологических пространств;

2) каждая функиия $\varphi \in W$ представляется рядом (2.11), который сходится в каждой точке области D;

3) система (1.4) является базисом Кете в $W$;

4) $S_{\Lambda}=0$ и для любого $m \geqslant 1$ существует $j \geqslant 1$ такой, что $\underline{h}_{\Psi_{j}} \geqslant H_{K_{m}}$;

5) $S_{\Lambda}=0$ и для любого $m \geqslant 1$ существует $\varphi \in I_{\Lambda}(D)$ такая, что $\underline{h}_{\varphi} \geqslant H_{K_{m}}$

6) $S_{\Lambda}=0$ и функиия $\Psi(z)$ имеет регулярный рост всюду в плоскости, $а$ ее верхний индикатор равен $H_{D}$, m. е. $\underline{h}_{\Psi} \equiv h_{\Psi} \equiv H_{D}$;

7) $S_{\Lambda}=0$ и существует функиия $\varphi \in I_{\Lambda}(\mathbb{C})$, которая имеет регулярный рост всюду в плоскости и ее верхний индикатор равен $H_{D}$, m..$\underline{h}_{\varphi} \equiv$ $h_{\varphi} \equiv H_{D}$.

ДокАЗАТЕЛЬСтво. Эквивалентность утверждений 1) и 2) доказана в предложении 2.13. Эквивалентность утверждений 2) и 3) вытекает из следствия 2.9. С учетом ограниченности $D$ эквивалентность утверждений 1$), 4$ ) и 5) следует из теорем $3.7,4.1,4.6$, следствия 4.8 и леммы 2.7 из [55]. Импликации 1) $\rightarrow 6$ ) и 6$) \rightarrow 7$ ) доказаны соответственно в следствии 4.9 и теореме 4.10 . Остается показать, что из утверждения 7) следует утверждение 1). Для этого достаточно установить, что выполнены условия теоремы 3.7. Поскольку $D$ ограничена, в проверке нуждается лиш условие 5) этой теоремы. Фиксируем $m \geqslant 1, \delta>0$ и покажем, что для некоторых $\varphi_{m} \in I_{\Lambda}(D)$ и $T>0$ имеет место включение $\lambda_{k} \in R_{m}^{\delta}$, если $\left|\lambda_{k}\right|>T$, где $R_{m}=\left\{z: \ln \left|\varphi_{m}(z)\right| \geqslant H_{K_{m}}(z)\right\}$.

Пусть $\varphi^{\prime}-$ произвольная не равная тождественно нулю функция из $I_{\Lambda}(D)$ (такая функция существует в силу нетривиальности $W)$ и $\varphi \in I_{\Lambda}(\mathbb{C})-$ функщия из утверждения 7). Положим $\tilde{\varphi}^{\prime}=\varphi^{\prime} / f_{\Lambda}$ и $\tilde{\varphi}=\varphi / f_{\Lambda}$. Как и выше, $\tilde{\varphi}^{\prime}$ и $\tilde{\varphi}$ целые, и для некоторых $a, b>0$ и любого $\alpha \in[0,1]$ верна оценка

$$
\psi_{\alpha}(z)=\alpha \ln \left|\tilde{\varphi}^{\prime}(z)\right|+(1-\alpha) \ln |\tilde{\varphi}(z)| \leqslant a+b|z|^{2}, \quad z \in \mathbb{C}
$$

Тогда из [53, теорема 5$]$ найдутся целая функция $\tilde{\varphi}_{\alpha}(z)$, постоянная $c>0$ и исключительное множество $E \subset \mathbb{C}$ такие, что

$$
|\ln | \tilde{\varphi}_{\alpha}(z)\left|-\psi_{\alpha}(z)\right| \leqslant c \ln |z|, \quad z \in \mathbb{C} \backslash E
$$

причем $E$ может быть покрыто кружками $B_{l}=\left\{z:\left|z-z_{l}\right|<r_{l}\right\}, l \geqslant 1$, такими, что $\sum r_{l}=A<\infty$. Положим $\varphi_{\alpha}=f_{\Lambda} \tilde{\varphi}_{\alpha}$. Фиксируем $\varepsilon \in\left(0, \alpha_{m} / 30\right)$, где $\alpha_{m}$ из неравенства (1.1). Выберем $\tau \in(0,1)$ такое, что для $F=\mathbf{S}$ и функций $h_{0}(z)=$ $H_{D}(z), h_{0}(z)=H_{D}(z)-\varepsilon|z|-3(3+\ln 48) \varepsilon|z|$ выполнено неравенство $(3.13)$, и $\delta_{1}>0, \delta_{1}<\min \{\delta / 8, \tau / 12\}$. По теореме об оценке снизу на окружностях целой 
функции конечного порядка и типа (см. [48, гл.I, §4, теорема 4.3]) существуют $d>0$ и последовательность $t_{j} \rightarrow+\infty$ такие, что

$$
t_{j+1}<\left(1+\delta_{1}\right) t_{j}, \quad \ln \left|\varphi^{\prime}(z)\right| \geqslant-d|z|^{2}, \quad|z|=t_{j}, \quad j \geqslant 1 .
$$

На окружности $\mathbf{S}$ выберем точки $y_{1}, \ldots, y_{s}$ так, что $\bigcup_{i=1}^{s} B\left(y_{i}, \delta_{1} / 2\right)$ содержит $\mathbf{S}$. Поскольку $\varphi$ имеет регулярный рост всюду в плоскости, то из $[55$, лемма 2.7] с учетом неравенства (3.13) для каждого $i=1, \ldots, s$ существует последовательность $\left\{y_{i, p}\right\}$ такая, что $\left|y_{i, p}\right| \uparrow+\infty,\left|y_{i, p} /\right| z_{i, p}\left|-y_{i}\right|<\delta_{1} / 2,\left|y_{i, p+1}\right| /\left|y_{i, p}\right|<$ $1+\delta_{1}$ и

$$
\left|y_{i, p}\right|>t_{1}, \quad \ln \left|\varphi\left(y_{i, p}\right)\right| \geqslant H_{D}\left(y_{i, p}\right)-\varepsilon\left|y_{i, p}\right|, \quad p \geqslant 1 .
$$

Нетрудно заметить, что для некоторого $T>0$ внешность круга $B(0, T)$ покрывается кругами $B\left(y_{i, p}, 2 \delta_{1}\left|y_{i, p}\right|\right), i=1, \ldots, s, p \geqslant 1$. Кроме того, из первых неравенств в (5.2) и (5.3) следует, что для любых $i=\overline{1, s}$ и $p \geqslant 1$ круг $B\left(y_{i, p}, \delta_{1}\left|y_{i, p}\right|\right)$ пересекает хотя бы одну из окружностей $|z|=t_{j}, j \geqslant 1$, скажем $|z|=t_{j_{i, p}}$. Можно считать, что $\delta_{1}\left|y_{i, p}\right|>4 A, i=1, \ldots, s, p \geqslant 1$, и так же, как в (3.11), выполнено неравенство

$$
\ln |\varphi(z)| \leqslant h_{\varphi}(z)+\varepsilon|z|=H_{D}(z)+\varepsilon|z|, \quad|z|>(1-\tau) T .
$$

Отсюда с учетом (3.13) имеем

$$
\ln |\varphi(z)| \leqslant H_{D}\left(y_{i, p}\right)+2 \varepsilon\left|y_{i, p}\right|, \quad z \in B\left(y_{i, p}, \tau\left|y_{i, p}\right|\right) .
$$

Используя это и (5.3), как и ранее, по теореме об оценке снизу модуля аналитической функции для любых $i=1, \ldots, s$ и $p \geqslant 1$ с учетом (3.13) получаем неравенство

$$
\begin{aligned}
\ln |\varphi(z)| & \geqslant H_{D}\left(y_{i, p}\right)-\varepsilon\left|y_{i, p}\right|-3(3+\ln 48) \varepsilon\left|y_{i, p}\right| \\
& \geqslant H_{D}(z)-2 \varepsilon|z|-3(3+\ln 48) \varepsilon|z| \geqslant H_{D}(z)-26 \varepsilon|z|
\end{aligned}
$$

которое выполнено в круге $B\left(y_{i, p}, 2 \delta_{1}\left|y_{i, p}\right|\right)$, но вне исключительных кружков $B_{k, i, p}, k=1, \ldots, k_{i, p}$, с суммой радиусов, равной $\delta_{1}\left|y_{i, p}\right| / 4$. Поскольку сумма диаметров кружков $B_{k, i, p}, \quad k=1, \ldots, k_{i, p}$, и $B_{l}, l \geqslant 1$, меньше $\delta_{1}\left|y_{i, p}\right|$, то неравенства (5.4) и (5.1) будут выполнены на некоторой окружности $S_{i, p}$, лежащей в кольце $B\left(y_{i, p}, 2 \delta_{1}\left|y_{i, p}\right|\right) \backslash B\left(y_{i, p}, \delta_{1}\left|y_{i, p}\right|\right)$. Выберем $\alpha_{0} \in(0,1)$ такое, что

$$
\alpha_{0}\left(H_{D}(z)-26 \varepsilon|z|\right) \leqslant \varepsilon|z| \quad \forall z, \quad \alpha_{0} d \leqslant \varepsilon .
$$

В силу (5.1), увеличивая при необходимости $T>0$, можно считать, что

$$
|\ln | \varphi_{\alpha_{0}}(z)\left|-\left(\alpha_{0} \ln \left|\varphi^{\prime}(z)\right|+\left(1-\alpha_{0}\right) \ln |\varphi(z)|\right)\right| \leqslant \varepsilon|z|, \quad z \in \mathbb{C} \backslash E, \quad|z|>(1-\tau) T .
$$

Пусть $z_{i, p} \in S_{i, p} \cap\left\{z:|z|=t_{j_{i, p}}\right\}$. Тогда с учетом неравенств (1.1), (5.2), (5.4), (5.5) и выбора числа $\varepsilon$ получаем

$$
\ln \left|\varphi_{\alpha_{0}}\left(z_{i, p}\right)\right| \geqslant H_{D}\left(z_{i, p}\right)-29 \varepsilon\left|z_{i, p}\right| \geqslant H_{K_{m+1}}\left(z_{i, p}\right)-29 \varepsilon\left|z_{i, p}\right| \geqslant H_{K_{m}}\left(z_{i, p}\right) .
$$


Таким образом, если $\varphi_{m}=\varphi_{\alpha_{0}}$, то $z_{i, p} \in R_{m}, i=1, \ldots, s, p \geqslant 1$. Нетрудно видеть, что $B\left(y_{i, p}, 2 \delta_{1}\left|y_{i, p}\right|\right) \subset B\left(z_{i, p}, 8 \delta_{1}\left|z_{i, p}\right|\right)$. Поэтому (с учетом выбора $\left.\delta_{1}\right)$ внешность круга $B(0, T)$ покрывается кругами $B\left(z_{i, p}, \delta\left|z_{i, p}\right|\right), i=1, \ldots, s, p \geqslant 1$. Это означает, что $\lambda_{k} \in R_{m}^{\delta}$, если $\left|\lambda_{k}\right|>T$. Остается установить включение $\varphi_{m} \in I_{\Lambda}(D)$. По построению $\varphi_{m}$ обрашается в нуль в точках $\lambda_{k}$ с кратностью, не меньшей, чем $m_{k}$. Кроме того, используя (5.6), принцип максимума, неравенство (3.13) и то, что $h_{\varphi^{\prime}}(z)<H_{D}(z), z \neq 0$, имеем

$$
h_{\varphi_{m}}(z) \leqslant \alpha_{0} h_{\varphi^{\prime}}(z)+\left(1-\alpha_{0}\right) H_{D}(z)<H_{D}(z), \quad z \neq 0 .
$$

Таким образом, $\varphi_{m} \in I_{\Lambda}(D)$. Теорема полностью доказана.

В заключение приведем условия, эквивалентные существованию функции $\varphi \in I_{\Lambda}(\mathbb{C})$ такой, что $\underline{h}_{\varphi} \equiv h_{\varphi} \equiv H_{D}$. В теории целых функций существует понятие асимптотического равенства. Говорят, что $f$ и $\tilde{f}$ асимптотически равны (по отношению к порядку один), т. е. $f \approx \tilde{f}$, если вне некоторого $C^{0}$-множества $\lim _{|z| \rightarrow \infty}|z|^{-1}(f(z)-\tilde{f}(z))=0$. Напомним, что $C^{0}$-множество - это множество, которое может быть покрыто кругами $\widetilde{B}_{j}=B\left(y_{j}, \tilde{r}_{j}\right)$ такими, что

$$
\lim _{r \rightarrow+\infty} r^{-1} \sum_{\left|y_{j}\right|<r} \tilde{r}_{j}=0
$$

В работе [57] показано, что $\widetilde{B}_{j}$ можно считать попарно непересекающимися. По аналогии с этим будем говорить, что $\psi$ - асимптотически субгармоническая функция, если существует субгармоническая функция $\tilde{\psi}$ такая, что $\psi \approx \tilde{\psi}$.

Теорема 5.5. Пусть $D$ - ограниченная выпуклая область и $\Lambda=\left\{\lambda_{k}, m_{k}\right\}$. Следующие утверждения әквивалентны:

1) существует функция $\varphi \in I_{\Lambda}(\mathbb{C})$ такая, ито $\underline{h}_{\varphi} \equiv h_{\varphi} \equiv H_{D}$;

2) $H_{D}(z)-\ln \left|f_{\Lambda}(z)\right|-$ асимптотически субгармоническая функиия.

ДоказАтельство. 1) $\rightarrow$ 2). Равенство $\underline{h}_{\varphi} \equiv h_{\varphi} \equiv H_{D}$ означает, что функция $\varphi$ имеет регулярнњй рост всюду в плоскости, или, другими словами, ее нулевое множество правильно распределено. Тогда (см., например, [48, гл. I, теорема 6.2]) $\ln |\varphi| \approx H_{D}$. Отсюда следует, что

$$
\ln |\varphi|-\ln \left|f_{\Lambda}\right| \approx H_{D}-\ln \left|f_{\Lambda}\right|
$$

Поскольку $\varphi \in I_{\Lambda}(\mathbb{C})$, то $\ln |\varphi|-\ln \left|f_{\Lambda}\right|-$ субгармоническая функция. Поэтому функция $H_{D}(z)-\ln \left|f_{\Lambda}(z)\right|$ асимптотически субгармоническая.

$2) \rightarrow 1)$. Пусть $\tilde{\psi}$ - субгармоническая функция такая, что $H_{D}-\ln \left|f_{\Lambda}\right| \approx \tilde{\psi}$. Тогда $H_{D} \approx \ln \left|f_{\Lambda}\right|+\tilde{\psi}$. Поскольку $\widetilde{B}_{j}$ попарно не пересекаются и $t_{j} /\left|y_{j}\right| \rightarrow 0$ при $j \rightarrow \infty$, то отсюда, из неравенства (3.13) и принципа максимума для субгармонических функций легко следует неравенство

$$
\ln \left|f_{\Lambda}(z)\right|+\tilde{\psi}(z) \leqslant a+H_{D}(z)+\varepsilon(z)|z| \quad \forall z
$$


где $\varepsilon(z) \rightarrow 0$, когда $|z| \rightarrow \infty$. Используя опять принцип максимума и $(4.7)$, как и ранее, устанавливаем, что $\tilde{\psi}$ удовлетворяет оценке (4.8). Тогда в силу [53, теорема 5] найдутся целая функция $\tilde{\varphi}, C>0$ и исключительное множество $E \subset \mathbb{C}$ такие, что

$$
|\ln | \tilde{\varphi}(z)|-\tilde{\psi}(z)| \leqslant C \ln |z|, \quad z \in \mathbb{C} \backslash E,
$$

причем множество $E$ может быть покрыто кружками $B_{i}=B\left(z_{i}, r_{i}\right), i \geqslant 1$, такими, что $\sum r_{i}=A<\infty$. Положим $\varphi(z)=\tilde{\varphi}(z) f_{\Lambda}(z)$. Очевидно, что объединение всех кругов $\widetilde{B}_{j}$ и $B_{i}$ является $C^{0}$-множеством. Тогда с учетом неравенства (5.7) имеем $\ln |\varphi| \approx H_{D}$. Отсюда, как и выше, следует, что $h_{\varphi} \leqslant H_{D}$, и, кроме того, неравенство $\underline{h}_{\varphi} \geqslant H_{D}$. Действительно, если последнее неверно, то для функции $\varphi$ имеет место оценка (4.21). Но это невозможно, так как объединение кругов $B\left(t_{r} y, \delta t_{r}\right), r \geqslant 1$, является множеством линейной плотности, не меньшей, чем $\delta$ (т.е. $\varlimsup_{t \rightarrow+\infty} t^{-1} \sum_{\left|t_{r}\right|<t} t_{r} \delta \geqslant \delta$ ), а потому не может лежать ни в каком $C^{0}$-множестве. Таким образом, $\underline{h}_{\varphi} \equiv h_{\varphi} \equiv H_{D}$. Теорема доказана.

ЗАмечАниЕ 5.6. Пусть $M_{\Lambda}(X)$ - сумма кратностей $m_{k}$ точек $\lambda_{k}$, попавших в множество $X$. Для компакта $K \subset \mathbb{C}$ через $K(\sigma)$ обозначим его $\sigma$-вздутие. Следуя работе [44], положим

$$
d_{\Lambda}(K)=\lim _{\sigma \rightarrow 0} \varlimsup_{t \rightarrow+\infty} t^{-1} M_{\Lambda}(t K(\sigma))
$$

Основываясь на результатах работ [33] и [44], можно показать, что утверждение 1) теоремы 5.5 эквивалентно следующему неравенству: $d_{\Lambda}(K) \leqslant \mu(K) \forall K \subset \mathbb{C}$. Здесь $\mu$ - риссовская мера субгармонической функции $H_{D}$.

\section{§ 6. Некоторые примеры}

ПримеР 6.1. Пусть $D, \widetilde{D}$ - выпуклые области и $K$ - выпуклый компакт в $\mathbb{C}$ такие, что $D=\widetilde{D}+K ; \Lambda=\left\{\lambda_{k}, m_{k}\right\}$; функция $\varphi \in I_{\Lambda}(D)$ имеет индикатор $h_{\varphi}$, равный $H_{K}$, и регулярный рост всюду в плоскости $\left(\underline{h}_{\varphi} \equiv h_{\varphi}\right) ; S_{\Lambda}=0$. Этих условий достаточно для разрешимости интерполяционной задачи. Действительно, пусть $\widetilde{K}_{m}$ - последовательность выпуклых компактов из $\widetilde{D}$, исчерпывающая $\widetilde{D}$. Тогда $K_{m}=K+\widetilde{K}_{m}$ - последовательность выпуклых компактов из $D$, исчерпываюшая $D$. Для каждого $m \geqslant 1$, как и вьше, найдем целую функцию $\tilde{\varphi}_{m}$ такую, что $\ln \left|\tilde{\varphi}_{m}\right|$ аппроксимирует $H_{\widetilde{K}_{m}}$. Положим $\varphi_{m}=\tilde{\varphi}_{m} \varphi$. Можно показать, что

$$
\underline{h}_{\varphi_{m}} \equiv h_{\varphi_{m}} \equiv h_{\varphi}+h_{\tilde{\varphi}_{m}} \equiv H_{K}+H_{\widetilde{K}_{m}} \equiv H_{K_{m}}
$$

Отсюда и из теоремы 5.1 следует тогда, что оператор $\Sigma: \mathbf{P}_{D} / I_{\Lambda}(D) \rightarrow \Phi-$ изоморфизм.

Обратно, пусть $D$ - ограниченная выпуклая область и $K$ - выпуклый компакт в $\mathbb{C} ; \Lambda=\left\{\lambda_{k}, m_{k}\right\}, m(\Lambda)=0, S_{\Lambda}=0 ;$ функция $\varphi \in I_{\Lambda}(D)$ имеет индикатор $h_{\varphi}$, равный $H_{K}$, и регулярный рост всюду в плоскости. Предположим, что оператор $\Sigma: \mathbf{P}_{D} / I_{\Lambda}(D) \rightarrow \Phi-$ изоморфизм. Тогда $D$ необходимо имеет вид $D=\widetilde{D}+K$, где $\widetilde{D}$ - выпуклая область. Действительно, согласно следствию 4.9 и теореме 4.10 сушествует функция $f \in I_{\Lambda}(\mathbb{C})$ такая, что $\underline{h}_{f} \equiv h_{f} \equiv H_{D}$. Положим $\tilde{\varphi}=f / \varphi$. Функция $\tilde{\varphi}$ целая, а из регулярности роста $\varphi$ вытекает тождество $h_{\varphi}+h_{\tilde{\varphi}} \equiv H_{D}$. Это означает, что $D=\widetilde{D}+K$, где $\widetilde{D}$ - внутренность сопряженной диаграммы $\tilde{\varphi}$. 
ПРИмеР 6.2. Пусть $D$ - ограниченная выпуклая область, $\Lambda=\left\{\lambda_{k}, m_{k}\right\}$, где $\lambda_{2 k}=k, \lambda_{2 k+1}=-k$ и $m_{k}=1, k \geqslant 1$. Последовательность $\left\{\lambda_{k}\right\}$ является множеством нулей целой функции экспоненциального типа $\varphi(z)=\sin (\pi z)$ (считаем, что $\left.\varphi \in I_{\Lambda}(D)\right)$. Эта функция имеет регулярный рост всюду в комплексной плоскости, а ее сопряженная диаграмма $K$ представляет собой отрезок мнимой оси $[-i, i]$, т. е. $H_{K}(z)=h_{\varphi}(z)=|\operatorname{Im} z|$. Кроме того, легко показать, что $Q_{\lambda}=0$. Таким образом, в силу сказанного выше оператор $\Sigma: \mathbf{P}_{D} / I_{\Lambda}(D) \rightarrow \Phi$ будет изоморфизмом тогда и только тогда, когда $D=\widetilde{D}+K$ для некоторой вьпуклой области $\widetilde{D}$. Последнее эквивалентно тому, что множества $\Gamma_{D}(-1)$ и $\Gamma_{D}(1)$ содержат отрезки длины не меньше, чем два. Действительно, нетрудно показать, что в этом случае в качестве $\widetilde{D}$ нужно взять множество, состоящее из середин вертикальных отрезков длины два, лежаших в $D$.

Пример 6.3. Пусть $D$ - ограниченная выпуклая область, $\Lambda=\left\{\lambda_{k}, m_{k}\right\}$, где $\lambda_{2 k}=k, \lambda_{2 k+1}=k+\varepsilon(k), \varepsilon(k) \rightarrow 0$, и $m_{k}=1, k \geqslant 1$. Последовательность $\left\{\lambda_{k}\right\}$ является частью нулей целой функции экспоненциального типа $f=\varphi \varphi_{1}$, где $\varphi-$ та же, что и в примере 6.2 , а $\varphi_{1}$ обладает свойствами, схожими со свойствами $\varphi$ (считаем, что $\left.f \in I_{\Lambda}(D)\right)$. Функция $f$ имеет регулярный рост всюду в комплексной плоскости. Если множества $\Gamma_{D}(-1)$ и $\Gamma_{D}(1)$ содержат отрезки длины не меньше, чем четыре, то $D=\widetilde{D}+K$, где $K$ - сопряженная диаграмма $f$. Таким образом, необходимым и достаточным условием того, что оператор $\Sigma: \mathbf{P}_{D} / I_{\Lambda}(D) \rightarrow \Phi-$ изоморфизм, является в этом случае равенство $S_{\Lambda}=0$, или, что то же самое, равенство $Q_{\Lambda}=0$. Имеем (при больших $k$ )

$$
\begin{aligned}
0 \leqslant & Q_{\Lambda}=\lim _{\delta \rightarrow 0} \varlimsup_{k \rightarrow \infty}\left|\lambda_{k}\right|^{-1} \int_{0}^{\delta\left|\lambda_{k}\right|} \frac{M_{\Lambda}\left(\lambda_{k}, t /\left|\lambda_{k}\right|\right)-m_{k}}{t} d t \\
= & \lim _{\delta \rightarrow 0} \varlimsup_{k \rightarrow \infty}\left|\lambda_{k}\right|^{-1} \int_{1 / 2}^{\delta\left|\lambda_{k}\right|} \frac{M_{\Lambda}\left(\lambda_{k}, t /\left|\lambda_{k}\right|\right)-m_{k}}{t} d t \\
& \quad+\lim _{\delta \rightarrow 0} \varlimsup_{k \rightarrow \infty}\left|\lambda_{k}\right|^{-1} \int_{0}^{1 / 2} \frac{M_{\Lambda}\left(\lambda_{k}, t /\left|\lambda_{k}\right|\right)-m_{k}}{t} d t \\
\leqslant & \lim _{\delta \rightarrow 0} \varlimsup_{k \rightarrow \infty}\left|\lambda_{k}\right|^{-1} \int_{1 / 2}^{\delta\left|\lambda_{k}\right|} \frac{3 t}{t} d t+\lim _{\delta \rightarrow 0} \varlimsup_{k \rightarrow \infty}\left|\lambda_{k}\right|^{-1} \int_{\varepsilon(k)}^{1 / 2} \frac{1}{t} d t \\
= & \varlimsup_{k \rightarrow \infty}\left|\lambda_{k}\right|^{-1}(-\ln (\varepsilon(k))) .
\end{aligned}
$$

Следовательно, равенство $Q_{\Lambda}=0$ будет выполнено в том и только том случае, когда $\varepsilon(k)=\exp \left(-\tilde{\varepsilon}(k)\left|\lambda_{k}\right|\right)$, где $\tilde{\varepsilon}(k)>0$ и $\tilde{\varepsilon}(k) \rightarrow 0$ при $k \rightarrow \infty$.

Пример 6.4. Выше мы показали, что в случае ограниченной выпуклой области разрешимость интерполяционной задачи (при условии $m(\Lambda)=0$ ) влечет за собой существование функции $\varphi \in I_{\Lambda}(\mathbb{C})$, которая имеет регулярный рост всюду в плоскости. В случае неограниченной области такой функции может не существовать. В подтверждение этого рассмотрим пример из работы [46]. Пусть $D-$ полуплоскость $\{z: \operatorname{Re} z<a\}$ и последовательность $\Lambda=\left\{\lambda_{k}, m_{k}\right\}$ - такая, что $m_{k} \equiv 1, \lambda_{k}=k$ при $k=2^{s}, s \geqslant 1$, а остальные $\lambda_{k}$ лежат на интервалах $\left(2^{s}, 2^{s+1}\right)$ так, что число $\lambda_{k}$, попавших на $\left(2^{s}, r\right), r<2^{s}$, равно целой части $\sqrt{r 2^{s}}$. В работе [46] доказано, что соответствующая интерполящионная задача разрешима. 
При этом, однако, не существует функции $\varphi \in I_{\Lambda}(\mathbb{C})$, которая имеет регулярньй рост на положительной вещественной полуоси. Действительно, предположим, что это не так, т. е. для некоторой $\varphi \in I_{\Lambda}(\mathbb{C})$ верно равенство $\underline{h}_{\varphi}(1)=h_{\varphi}(1)$. Тогда по лемме 2.7 из [55] сушествует последовательность $\left\{z_{j}\right\}$ такая, что $\left|z_{j}\right| \rightarrow+\infty$, $\left|z_{j} /\right| z_{j}|-1| \rightarrow 0,\left|z_{j+1}\right| /\left|z_{j}\right| \rightarrow 1$ и $\lim _{j \rightarrow \infty} \ln \left|\varphi\left(z_{j}\right)\right| /\left|z_{j}\right| \geqslant h_{\varphi}(1)$.

Пусть $\varepsilon>0$. Из последнего неравенства и леммы 3.2 получаем

$$
M_{\Lambda}\left(z_{j}, \frac{\delta}{4}\right) \leqslant 2 \varepsilon\left|z_{j}\right|, \quad j \geqslant j_{0},
$$

где $\delta>0$ выбрано из условия $h_{\varphi}(z) \leqslant h_{\varphi}(1)+\varepsilon, z \in B(1,2 \delta)$. Используя выпуклость $h_{\varphi}$, нетрудно показать, что для некоторого $c>0$ верно неравенство $h_{\varphi}(z) \leqslant h_{\varphi}(1)+c|z-1|, z \in B(1,1)$. Поэтому в (6.1) в качестве $\delta$ можно взять $\varepsilon /(2 c)$. Из свойств последовательности $\left\{z_{j}\right\}$ следует, что для некоторого $b>0$ луч $[b,+\infty)$ покрывается кругами $B\left(z_{j}, \varepsilon\left|z_{j}\right| /(16 c)\right), j \geqslant 1$. Выберем номер $j_{s}$ такой, что $2^{s} \in B\left(z_{j_{s}}, \varepsilon\left|z_{j_{s}}\right| /(16 c)\right), s \geqslant s_{0}$. Тогда интервал $\left(2^{s}, \varepsilon\left|z_{j_{s}}\right| /(16 c)\right)$ лежит в круге $B\left(z_{j_{s}}, \varepsilon\left|z_{j_{s}}\right| /(8 c)\right)$, а при малых $\varepsilon>0$ будет выполнено неравенство $2^{s} \geqslant\left|z_{j_{s}}\right| / 2, s \geqslant s_{0}$. Следовательно, по определению $\lambda_{k}$ имеем

$$
M_{\Lambda}\left(z_{j}, \frac{\varepsilon}{8 c}\right) \geqslant\left[\sqrt{\frac{2^{s} \varepsilon\left|z_{j_{s}}\right|}{16 c}}\right] \geqslant\left[\left|z_{j_{s}}\right| \sqrt{\frac{\varepsilon}{32 c}}\right] .
$$

При малых $\varepsilon>0$ это противоречит (6.1), где $\delta=\varepsilon /(2 c)$.

\section{Список литературы}

1. Напалков В. В. Уравнения свертки в многомерных пространствах. М.: Наука, 1982.

2. Лейхтвейс K. Выпуклые множества. М.: Наука, 1985.

3. Красичков-Терновский И. Ф. Однородное уравнение типа свертки на выпуклых областях // ДАН СССР. 1971. Т. 197. № 1. С. 29-31.

4. Красичков-Терновский И. Ф. Инвариантные подпространства аналитических функций. І. Спектральный анализ на выпуклых областях // Матем. сб. 1972. Т. 87. № 4. C. $459-489$.

5. Красичков-Терновский И. Ф. Инвариантные подпространства аналитических функций. II. Спектральный синтез на выпуклых областях // Матем. сб. 1972. Т. 88. № 1. C. $3-30$.

6. Valiron G. Sur les solutions des equations differentielles lineaires d'ordre infini et a coefficients constants // Ann. Sci. Ec. Norm. Sup. 1929. V. 46. № 1. P. 25-53.

7. Schwartz L. Theorie generale des fonctions moyenne-periodique // Ann. Math. 1947. V. 48. № 4. P. 857-929.

8. Гельфонд А. О. Линейные дифференциальные уравнения с постоянными коэффициентами бесконечного порядка и асимптотические периоды целых функций // Тр. Матем. ин-та им. В.А. Стеклова АН СССР. 1951. Т. 38.

9. Dickson D. G. Expansions in series of solutions of linear difference-differential and infinite order differential equations with constant coefficients // Memor. Amer. Math. Soc. 1957. V. 23. P. 1-72.

10. Гельфонд А. О. Исчисление конечных разностей. М.: Наука, 1967.

11. Гончаров В. Л. Теория интерполирования и приближения функций. М.: Гостехиздат, 1954.

12. Евграфов М. А. Интерполяционная задача Абеля-Гончарова. М.: Гостехиздат, 1954.

13. Левин Б. Я. О некоторых приложениях интерполяционного ряда Лагранжа к теории целых функций // Матем. сб. 1940. Т. 8. № 3. С. 437-454. 
14. Леонтьев $A . \Phi$. Об интерполировании в классе целых функций конечного порядка // ДАН СССР. 1948. Т. 61. № 5. С. 785-787.

15. Леонтьев А. Ф. Об интерполировании в классе целых функций конечного порядка нормального типа // ДАН СССР. 1949. Т. 66. № 2. С. 153-156.

16. Леонтьев А.Ф. Об интерполяционной задаче // ДАН СССР. 1949. Т. 66. № 3. C. $331-334$.

17. Леонтьев $A . \Phi . \mathrm{K}$ вопросу об интерполировании в классе целых функций конечного порядка // Матем. сб. 1957. Т. 41. № 1. С. 81-96.

18. Леонтьев A. Ф. О значениях целой функции конечного порядка в заданных точках // Изв. АН СССР. Сер матем. 1958. Т. 22. № 3. С. 387-394.

19. Ибрагимов И. И. Методы интерполяции функций и некоторые их приложения. М.: Наука, 1971.

20. Ибрагимов И. И., Келдыш М. В. Об интерполяции целых функций // Матем. сб. 1947. T. 20. № 2. C. 283-292.

21. Казьмин Ю. А. К вопросу о восстановлении аналитической функции по ее элементам // Изв. АН СССР. Сер матем. 1966. Т. 30. № 2. С. 307-324.

22. Казьмин Ю. А. Об одной интерполяционной задаче // Сиб. матем. журн. 1967. Т. 8. № 2. C. 293-312.

23. Казьмин Ю. А. Об одной интерполяционной задаче // Сиб. матем. журн. 1967. Т. 8. № 3. C. 587-600.

24. Коробейник Ю. Ф. Интерполяционные задачи, нетривиальные разложения нуля и представляющие системы // Изв. АН СССР. Сер. матем. 1980. Т. 44. № 5. С. 1066-1144.

25. Коробейник Ю. Ф. Представляющие системы // УМН. 1981. Т. 1. С. 73-126.

26. Левин Б. Я. Распределение корней целых функций. М.: Гостехиздат, 1956.

27. Mursi M., Winn E. On the interpolated integral function of given order // Quart. J. Math. 1933. V. 4. P. $173-179$.

28. Macintyre A. J., Wilson R. On the order of the interpolated integral functions // Quart. J. Math. 1933. V. 5. P. 211-220.

29. Фирсакова О.С. Некоторые вопросы интерполирования с помощью целых функций // ДАН СССР. 1958. Т. 120. № 3. С. 477-480.

30. Малютин К. Г. Интерполяция голоморфными функциями: Дис. .. канд. физ.-матем. наук. Харьков, 1980.

31. Малютин К. Г. Об интерполяционной задаче в классе целых функций вполне регулярного роста // Зап. науч. сем. ЛОМИ. 1984. Т. 135. С. 96-107.

32. Руссаковский A. М. Об интерполяции в классе целых функций, имеющих индикатор не выше данного // Теория функций, функц. анализ. и их прилож. Вып. 37. Харьков: Вища школа, 1982. С. 111-114.

33. Грииин А.Ф. О множествах регулярного роста целых функций // Теория функций, функц. анализ и их прилож. Вып. 40. Харьков: Вища школа, 1983. С. 36-47.

34. Гришин A.Ф. О множествах регулярного роста целых функций // Теория функций, функц. анализ и их прилож. Вып. 41. Харьков: Вища школа, 1984. С. 39-55.

35. Гришин A. . О множествах регулярного роста целых функций // Теория функций, функц. анализ и их прилож. Вып. 42. Харьков: Вища школа, 1984. С. 37-43.

36. Mursi M. Sur l'order de fonctions entieres definies par interpolation // Bull. Sci. Math. 1949. V. 73. P. $96-112$.

37. Лапин Г. П. О целых функциях конечного порядка, принимающих вместе с производными заданные значения в заданных точках // Сиб. матем. журн. 1965. Т. 6. №6. C. $1267-1281$.

38. Лапин Г. П. Интерполирование в классе целых функций конечного порядка // Изв. вузов. Математика. 1959. Т. 5. С. 146-153.

39. Братищев А.В. Об интерполяционной задаче в некоторых классах целых функций // Сиб. матем. журн. 1976. Т. 7. № 1. С. 30-44.

40. Братищев А.В. О разрешимости интерполяционной задачи в классах $[\rho(r), H(\theta))$, $[\rho(r), H(\theta)] / /$ Механика сплошной среды. Ростов-на-Дону. 1981. С. 49-54. 
41. Братищев А.В., Коробейник Ю. Ф. Интерполяционная задача в пространствах целых функций конечного порядка // Изв. АН СССР. Сер. матем. 1976. Т. 40. № 5. C. $1102-1127$.

42. Руссаковский A. М. Об интерполяции в классе целых функций, имеющих индикатор не выше данного // Теория функций, функц. анализ и их прилож. Вып. 37. Харьков: Вища школа, 1982. С. 111-114.

43. Руссаковский $A$. М. Об интерполяции в классе целых функций, имеющих индикатор не выше данного // Теория функций, функц. анализ и их прилож. Вып. 41. Харьков: Вища школа, 1984. С. 119-122.

44. Гришин A. Ф., Руссаковский A. М. Свободная интерполяция целыми функциями // Теория функций, функц. анализ и их прилож. Вып. 44. Харьков: Вища школа, 1985. С. 32-42.

45. Братищев $A . B$. Один тип оценок снизу целых функций конечного порядка и некоторые приложения // Изв. АН СССР. Сер. матем. 1984. Т. 48. № 3. С. 451-475.

46. Братищев А.В. Базисы Кете, целые функции и их приложения: Дис. ... докт. физ.-матем. наук. Ростов-на-Дону, 1993.

47. Berenstein C. A., Taylor B. A. A new look at interpolation theory for entire functions of one variables // Adv. Math. 1979. V. 33. № 2. P. 109-143.

48. Леонтьев А. Ф. Целые функции. Ряды экспонент. М.: Наука, 1983.

49. Робертсон А. П., Робертсон В. Дж. Топологические векторные пространства. М.: Мир, 1967.

50. Гротендик А. О. О пространствах $(F)$ и $(D F)$ // Математика. 1958. Т. 2. № 3 . C. $81-127$.

51. Хермандер Л. Анализ линейных дифференциальных операторов с частными производными. I. Теория распределений и анализ Фурье. М.: Мир, 1986.

52. Ронкин Л. И. Введение в теорию целых функций многих переменных. М.: Наука, 1971.

53. Юлмухаметов Р. С. Аппроксимация субгармонических функций // Anal. Math. 1985. T. 11. C. 257-282.

54. Кривошеев A. C., Напалков В. В. Комплексный анализ и операторы свертки // УМН. 1992. T. 47. №6. C. 3-58.

55. Кривошеев A. C. Об индикаторах целых функций и продолжении решений однородного уравнения свертки // Матем. сб. 1993. Т. 184. № 8. С. 81-108.

56. Кривошеев A.C. Аналитическое продолжение функций из инвариантных подпространств в выпуклых областях комплексного пространства // Изв. РАН. Сер. матем. 1998. Т. 62. № 2. С. $75-102$.

57. Красичков-Терновский И. Ф. Одна геометрическая лемма, полезная в теории целых функций, и теоремы типа Левинсона // Матем. заметки. 1978. Т. 24. № 4. С. 531-546.

Поступило в редакцию 